\title{
Measurement of the W Boson Helicity In Top-Antitop QuARK Events WITH THE CDF II EXPERIMENT
}

Thorsten Chwalek

\section{DiPlOMA THESIS}

AT THE FACULTy FOR PHYSICS

OF THE University OF KARLSRUhe (TH)

Referee: Prof. Dr. Th. Müller

Institut für Experimentelle Kernphysik

Co-Referee: Priv.-Doz. Dr. W. Wagner Institut für Experimentelle Kernphysik 



\section{Introduction}

In 1995 the top quark was discovered at the Tevatron proton-antiproton collider at Fermilab by the CDF and $\mathrm{D} \varnothing$ collaborations $[1,2]$. It is the most massive known elementary particle and its mass is currently measured with a precision of about $1.3 \%[3,4]$. However, the measurements of several other top quark properties are still statistically limited, so the question remains whether the Standard Model of elementary particle physics successfully predicts these properties. This thesis addresses one interesting aspect of top quark decay, the helicity of the produced $W$ boson.

Until the start of the Large Hadron Collider (LHC) at CERN, the Tevatron with a center-of-mass energy of $\sqrt{s}=1.96 \mathrm{TeV}$ is the only collider, where top quarks can be produced. In the Standard Model the top quark decays predominantly into a $W$ boson and a $b$ quark, with a branching ratio close to $100 \%$. The $\mathrm{V}-\mathrm{A}$ structure of the weak interaction of the Standard Model predicts that the $W^{+}$bosons from the top quark decay $t \rightarrow W^{+} b$ are dominantly either longitudinally polarized or left handed, while right handed $W$ bosons are heavily suppressed and even forbidden in the limit of a massless $b$ quark.

Under the assumption of a massless $b$ quark, for a top quark mass of $173 \mathrm{GeV} / c^{2}$ the Standard Model predicts the fraction $F_{0}$ of longitudinally polarized $W$ bosons to be 0.7 and 0.3 for the fraction $F_{-}$of left handed $W$ bosons, while the fraction $F_{+}$of right handed $W$ bosons is predicted to be zero. Since next-to-leading order corrections change these fractions only slightly, a significant deviation from the predicted value for $F_{0}$ or a nonzero value for $F_{+}$could indicate new physics. Left-right symmetric models [5], for example, lead to a significant right handed fraction of $W$ bosons in top decays. Such a right handed component (V+A coupling) would lead to a smaller left handed fraction, while $F_{0}$ would remain unchanged. Since the decay rate to longitudinal $W$ bosons depends on the Yukawa coupling of the top quarks, the measurement of $F_{0}$ is sensitive to the mechanism of electroweak symmetry breaking. Alternative models can lead to an altered $F_{0}$ fraction.

In this analysis the $W$ helicity fractions are measured in a selected sample rich in $t \bar{t}$ events where one lepton, at least four jets, and missing transverse energy are required. All kinematic quantities describing the $t \bar{t}$ decay are determined. As a sensitive observable, we use the cosine of the decay angle $\theta^{*}$, which is defined as the angle between the momentum of the charged lepton in the $W$ boson rest frame and the $W$ boson momentum in the top quark rest frame. The data used in this analysis were taken with the Collider Detector at Fermilab (CDF II) in the years 2002 - 2006 and correspond to an integrated luminosity of about $955 \mathrm{pb}^{-1}$. 
Previous CDF measurements of the $W$ boson helicity fractions in top quark decays used either the square of the invariant mass of the charged lepton and the $b$ quark jet, $M_{\ell b}^{2}$, [6-8] or the lepton $p_{\mathrm{T}}$ distribution $[7,9]$ as a discriminant. The D $\varnothing$ collaboration used a matrix-element method to extract a value of $F_{0}$ [10]; in a second analysis the reconstructed distribution of $\cos \theta^{*}$ [11] was utilized to measure $F_{+}$. CDF gives the latest value of $F_{0}=0.74_{-0.34}^{+0.22}[7]$, while D $\varnothing$ measured $F_{0}=0.56 \pm 0.31[10]$. The CDF collaboration also gives the current upper limit of $F_{+}<0.09[8]$.

The thesis is organized as follows. In the first chapter a brief overview over the Standard Model of elementary particle physics with particular emphasis on the production and decay of top quarks is given. Also the theoretical aspects concerning the helicity of $W$ bosons from the top quark decay are discussed in this chapter. Chapter two describes the accelerator chain at Fermilab and the CDF II detector. The third chapter presents the data and Monte Carlo samples used in this analysis and illustrates the event selection of $t \bar{t}$ candidates in the lepton+jets channel. The full reconstruction of kinematic quantities of $t \bar{t}$ pairs is described in detail in chapter four. Chapter five presents the measurement of the $W$ boson helicity fractions. In chapter six the developed method is verified and the different sources of systematic uncertainties are discussed and quantified. Finally, the conclusion and an outlook are given in chapter seven. 


\section{Contents}

1 Theory 7

1.1 The Standard Model of Elementary Particle Physics . . . . . . . . . . 7

1.2 Top Quark Production and Decay . . . . . . . . . . . . . . . . . . 10

$1.3 W$ - Helicity in Top Quark Decays . . . . . . . . . . . . . . . . . 11

1.4 Sensitive Observable $\cos \theta^{*} \ldots \ldots \ldots \ldots$

2 The CDF II Experiment $\quad 17$

2.1 The Collider . . . . . . . . . . . . . . . . . . 17

2.2 The CDF II Detector . . . . . . . . . . . . . . . . . . . . . . 21

2.2.1 Tracking . . . . . . . . . . . . . . . . . 21

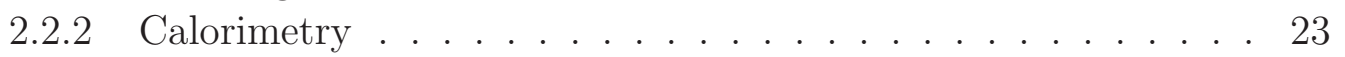

2.2.3 Muon Reconstruction . . . . . . . . . . . . . . . . . 24

2.2 .4 Trigger System . . . . . . . . . . . . . . . 24

3 Event Selection and Background Estimation $\quad 27$

3.1 Data Samples . . . . . . . . . . . . . . . . . . . 27

3.2 Monte Carlo Samples . . . . . . . . . . . . . . . . . . . . . . 28

3.2.1 Monte Carlo Generators . . . . . . . . . . . . . . . . . 29

3.2 .2 Used MC Samples . . . . . . . . . . . . . . . . . . . 30

3.3 Event Selection . . . . . . . . . . . . . . . . . . . . . 32

3.4 Background Estimation . . . . . . . . . . . . . . . . 37

4 Full Reconstruction of $t \bar{t}$ Events 39

4.1 Full Reconstruction . . . . . . . . . . . . . . . . . . . . . . . . . 39

4.2 Selection of one Event Interpretation . . . . . . . . . . . . . . 42

4.3 Best possible Event Interpretation in MC . . . . . . . . . . . . . 46

4.4 Performance of our Selection . . . . . . . . . . . . . . . . . . 48

4.5 Data - MC Comparison . . . . . . . . . . . . . . . 54

5 Measurement of the Helicity Fractions $\quad 61$

5.1 Measurement Strategy . . . . . . . . . . . . . . . . . . 61

5.2 Calculation of the normalized Signal Templates . . . . . . . . . . 64

5.3 Likelihood Fit . . . . . . . . . . . . . . . . . . . . . 76

5.4 Results . . . . . . . . . . . . . . . . . . . . . . . . . . . . . . . . . . . 77

5.5 Unfolded $\cos \theta^{*}$ Distribution . . . . . . . . . . . . . 78 
6 Verification of our Method and Systematic Uncertainties 81

6.1 Pseudo Experiments . . . . . . . . . . . . . . . . . 81

6.2 Verification of our Method . . . . . . . . . . . . . . . . . 82

6.2.1 Consistency Check . . . . . . . . . . . . . . . 82

6.2 .2 Sensitivity . . . . . . . . . . . . . . . . . 83

6.2.3 Further Checks . . . . . . . . . . . . . . . . . . . . 84

6.3 Systematic Uncertainties . . . . . . . . . . . . . . . 86

$\begin{array}{lll}7 & \text { Conclusion and Outlook } & 91\end{array}$ 


\section{List of Figures}

1.1 Feynman diagram of electron-electron scattering . . . . . . . . . . 9

1.2 Some leading-order Feynman diagrams of $t \bar{t}$ pair production . . . . . 10

1.3 Dependence of the helicity fractions on different anomalous form factor settings . . . . . . . . . . . . . . . 15

$1.4 W$ boson decay in the $W$ rest frame for different helicities . . . . . . 16

1.5 Theoretical $\cos \theta^{*}$ distribution for different helicities . . . . . . . . . . 16

2.1 Aerial shot of the Tevatron main ring. . . . . . . . . . . . . 17

2.2 Schematic view of Fermilab's accelerator chain for Run II . . . . . . . 18

2.3 Peak luminosity per store . . . . . . . . . . . . . . . . . . . 20

2.4 Delivered and recorded integrated luminosity . . . . . . . . . . . . . 20

2.5 The CDF II detector with its different components . . . . . . . . . . 21

2.6 Elevation view of one half of the CDF II detector . . . . . . . . . . . 22

2.7 Longitudinal view of the CDF II tracking system . . . . . . . . . . . 23

2.8 Dataflow of the CDF II trigger system . . . . . . . . . . . . 25

3.1 Schematic view of a secondary vertex . . . . . . . . . . . . 35

4.1 Feynman diagram of $t \bar{t}$ production and decay. . . . . . . . . . . . . . 39

4.2 Determination of the input parameters for the calculation of $\chi^{2}$. . . 44

4.3 Probabilities R and R . . . . . . . . . . . . . . . 45

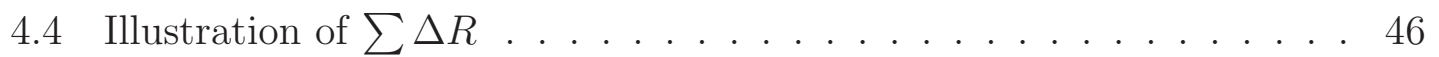

4.5 Illustration of $\sum \Delta R$ for all hypotheses of a certain event . . . . . . . 47

4.6 Performance of the hypothesis selection. . . . . . . . . . . . . . 48

4.7 Distributions of kinematic quantities of the leptonically decaying $W$ boson and the neutrino . . . . . . . . . . . . . . . . 50

4.8 Distributions of kinematic quantities of the hadronically decaying $W$ boson . . . . . . . . . . . . . . . . 51

4.9 Distributions of kinematic quantities of the semileptonically decaying top quark . . . . . . . . . . . . . . . . . . 52

4.10 Distributions of kinematic quantities of the hadronically decaying top

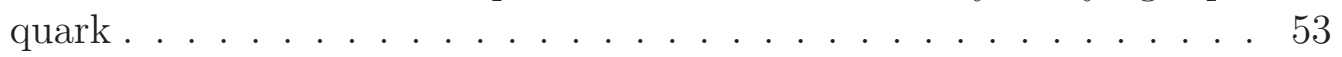

4.11 Data-MC comparison for $\Psi, \chi^{2}$, and $P_{\mathrm{b}-\text { light }} \ldots \ldots \ldots . . \ldots 55$

4.12 Data-MC comparison for $P_{\mathrm{T}}$ and $\eta$ of the charged lepton, the neutrino, and the leptonically decaying $W$ boson . . . . . . . . . . 56

4.13 Data-MC comparison for $P_{\mathrm{T}}, \eta$ and $m$ of the hadronically decaying $W$ boson . . . . . . . . . . . . . . . . . 57 
4.14 Data-MC comparison for $P_{\mathrm{T}}, \eta$ and $m$ of the semileptonically decaying top quark . . . . . . . . . . . . . . . . . . 5 58

4.15 Data-MC comparison for $P_{\mathrm{T}}, \eta$ and $m$ of the hadronically decaying top quark . . . . . . . . . . . . . . . . . . 59

5.1 Schematic view of the decay angle $\theta^{*} \ldots \ldots$. . . . . . . . . 61

5.2 Theoretical $\cos \theta^{*}$ distributions for different helicities . . . . . . . . . 62

5.3 Resolution of the full reconstruction . . . . . . . . . . . . . . . . . 62

5.4 Reconstructed $\cos \theta_{\text {rec }}^{*}$ distribution from data . . . . . . . . . . 63

5.5 Effects of event selection and reconstruction on the $\cos \theta^{*}$ distribution 65

5.6 Normalized efficiency for different helicities . . . . . . . . . . . . 67

5.7 Migration matrix elements for different helicities . . . . . . . . . . . . 69

5.8 Migration matrix elements for different helicities in 20 bins . . . . . . 70

5.9 Relative efficiency for SM helicity . . . . . . . . . . . . . . . . 72

5.10 Migration matrix elements for SM helicity . . . . . . . . . . 73

5.11 Fit templates for $F_{0}\left(F_{+}=0\right) \ldots \ldots \ldots \ldots$. . . . . . . . 74

5.12 Fit templates for $F_{+}\left(F_{0}=0.7\right) \ldots \ldots \ldots \ldots . \ldots \ldots$

5.13 Negative logarithm of the likelihood functions . . . . . . . . . . 77

5.14 Posterior probability density as a function of $F_{+} \ldots \ldots . . .78$

5.15 Unfolded $\cos \theta^{*}$ distributions in data . . . . . . . . . . . . . 79

6.1 Pull distributions for the consistency check . . . . . . . . . . . . 83

6.2 Expected sensitivity for the measurements of $F_{0}$ and $F_{+} \ldots \ldots$. . . 83

6.3 Pseudo experiments for different values of the helicity fractions . . . . 84

6.4 Pseudo experiments for different values of the helicity fractions means and widths . . . . . . . . . . . . . . . . . . . 85

7.1 Expected sensitivities for higher integrated luminosities . . . . . . . . 92 


\section{List of Tables}

1.1 Properties of the fermions (spin- $\frac{1}{2}$ particles $) \ldots \ldots \ldots$

1.2 Properties of the gauge bosons (spin-1 particles) . . . . . . . . 8

1.3 Branching ratios for the three different topologies of $t \bar{t}$ events . . . . . 11

2.1 CDF II calorimeter properties . . . . . . . . . . . . . . . . . 24

2.2 Muon system properties . . . . . . . . . . . . . . . . . . . 24

3.1 Used data samples . . . . . . . . . . . . . . . . . . . . . . . 28

3.2 Used Monte Carlo samples . . . . . . . . . . . . . . . . . . . . . . 31

3.3 Cut flow table of $t \bar{t}$ event selection . . . . . . . . . . . . . 36

3.4 Yield of $t \bar{t}$ candidates in the CDF data . . . . . . . . . . . . 37

3.5 Summary of background events . . . . . . . . . . . . . 38

4.1 Quality of the hypothesis selection . . . . . . . . . . . . 49

6.1 Summary of systematic uncertainties . . . . . . . . . . . . . . 89 


\section{Chapter 1}

\section{Theory}

In this chapter the theoretical framework of particle physics, the Standard Model, is briefly described. Also the mechanism of production and decay of the top quark is described. In more detail we discuss the theoretical aspects of the top quark decay in polarized $W$ bosons.

\subsection{The Standard Model of Elementary Particle Physics}

The Standard Model of elementary particle physics (SM) has been very successful in the last decades in describing the fundamental particles and their interactions $[12,13]$. In the SM the basic constituents of matter are spin $\frac{1}{2}$ particles, the fermions, while all interactions between these fermions are mediated by spin 1 particles, the gauge bosons.

Up to now we know twelve different fermions, six quarks and six leptons, along with their antiparticles. The six different quark flavors are up $(u)$, down $(d)$, charm $(c)$, strange $(s)$, top $(t)$, and bottom $(b)$. Quarks carry electric charge of either $-\frac{1}{3}$ $(d, s, b)$ or $+\frac{2}{3}(u, c, t)$ of the elementary charge. Since quarks also underly the strong interaction, they carry an additional charge, called color charge, which can be of three types (red, blue or green). Since free colored particles are not observed, the quarks must be confined into colorless particles. Baryons consist of three quarks, where each quark has a different color, which leads in total to a colorless (or "white") object. For example the proton is formed from two $u$ quarks and one $d$ quark. Mesons consist of one quark carrying a certain color and an antiquark which carries the corresponding anticolor, as for example the positive charged pion $\pi^{+}$is made up of one $u$ and one $\bar{d}$ quark. The other six fundamental particles, the leptons, do not partake in the strong interaction and carry therefore no color charge. There are three charged leptons, the electron $e$, the muon $\mu$, and the tau $\tau$, and the corresponding neutral neutrinos $\nu_{e}, \nu_{\mu}, \nu_{\tau}$.

The six quarks as well as the six leptons are ordered in three so-called families or 
generations. To each mentioned particle corresponds one antiparticle, which has the same properties, but opposite charge. The SM predicts the existence of these twelve fundamental particles, but makes no statement on the masses of the particles. The three generations of fundamental particles are shown in table 1.1 together with their electric charge and mass.

\begin{tabular}{|lcrr|}
\hline name & symbol & el. charge & mass \\
\hline \hline up quark & $u$ & $\frac{2}{3}$ & $(1.5-3.0)$ \\
down quark & $d$ & $-\frac{1}{3}$ & $(3.0-7.0)$ \\
electron & $e$ & -1 & 0.511 \\
$e$-neutrino & $\nu_{e}$ & 0 & $<2 \cdot 10^{-6}$ \\
\hline \hline charm quark & $c$ & $\frac{2}{3}$ & $(1.25 \pm 0.09) \cdot 10^{3}$ \\
strange quark & $s$ & $-\frac{1}{3}$ & $(95 \pm 25)$ \\
muon & $\mu$ & -1 & 106 \\
$\mu$-neutrino & $\nu_{\mu}$ & 0 & $<0.190$ \\
\hline \hline top quark & $t$ & $\frac{2}{3}$ & $(172.5 \pm 2.3) \cdot 10^{3}$ \\
bottom quark & $b$ & $-\frac{1}{3}$ & $(4.20 \pm 0.07) \cdot 10^{3}$ \\
tau & $\tau$ & -1 & 1777 \\
$\tau$-neutrino & $\nu_{\tau}$ & 0 & $<18.2$ \\
\hline
\end{tabular}

Table 1.1: Properties of the fermions (spin- $\frac{1}{2}$ particles) [14]. The electric charge is given in units of the electron charge, the mass in units of $\mathrm{MeV} / c^{2}$.

Up to now four fundamental forces are known, three of them are described by the SM, the electromagnetic force, described by the theory of Quantum Electro Dynamics (QED), the weak force, and the strong force, described by the theory of Quantum Chromo Dynamics (QCD). The fourth force, gravitation, is not described within the SM, but by the theory of General Relativity. In the SM the forces are mediated by special particles, called gauge bosons since the theories that describe these forces are all gauge theories. The electromagnetic force is mediated by chargeand massless photons. Massless gluons are the gauge bosons of the strong force, while the weak force is mediated by massive $W$ and $Z$ bosons. The properties of the gauge bosons are presented in table 1.2.

\begin{tabular}{|lcccr|}
\hline name & force & symbol & el. charge & mass \\
\hline \hline gluon & strong & $\mathrm{g}$ & 0 & 0 \\
\hline photon & electromagnetic & $\gamma$ & 0 & 0 \\
\hline W boson & weak & $W^{ \pm}$ & \pm 1 & $80.403 \pm 0.029$ \\
Z boson & weak & $Z^{0}$ & 0 & $91.188 \pm 0.002$ \\
\hline
\end{tabular}

Table 1.2: Properties of the gauge bosons (spin-1 particles) [14]. The electric charge is given in units of the electron charge, the mass in units of $\mathrm{GeV} / c^{2}$. 
The only particle predicted by the SM not yet discovered is the so-called Higgs boson [15]. Since all theories in the SM are gauge theories the corresponding gauge bosons should be massless. To explain the mass of the massive bosons of the weak interaction one has to introduce an additional scalar field, the Higgs field. Particles then get their masses by interacting with that field. The quantum of the Higgs field is the Higgs boson, which is also massive due to its selfinteraction.

Another important fact concerning the weak interaction besides its massive gauge bosons is that the eigenstates of the weak interaction are not the same as the mass eigenstates of the quarks. In electroweak processes one therefore has to transform the different eigenstates into each other, which is done by the Cabibbo-KobayashiMaskawa (CKM) matrix [16,17].

$$
\left(\begin{array}{c}
d^{\prime} \\
s^{\prime} \\
b^{\prime}
\end{array}\right)=\left(\begin{array}{lll}
V_{u d} & V_{u s} & V_{u b} \\
V_{c d} & V_{c s} & V_{c b} \\
V_{t d} & V_{t s} & V_{t b}
\end{array}\right)\left(\begin{array}{c}
d \\
s \\
b
\end{array}\right)
$$

The coupling of two quarks $q_{1}$ and $q_{2}$ to a $W$ boson is proportional to the matrix element $V_{q_{1} q_{2}}$. The values for the magnitudes of the matrix elements are [14]:

$$
\left(\begin{array}{ccc}
0.9739 \text { to } 0.9751 & 0.221 \text { to } 0.227 & 0.0029 \text { to } 0.0045 \\
0.221 \text { to } 0.227 & 0.9730 \text { to } 0.9744 & 0.039 \text { to } 0.044 \\
0.0048 \text { to } 0.014 & 0.037 \text { to } 0.043 & 0.9990 \text { to } 0.9992
\end{array}\right)
$$

Only particles that carry the charge corresponding to a certain force, can interact via that force. The interaction between two charged particles takes place by emission and reabsorption of gauge bosons. As visualization of such processes serve so-called Feynman diagrams, which are two dimensional space-time diagrams. Following the Feynman rules, one can translate diagrams for a specific physical process into the corresponding formula. Only fundamental particles appear in Feynman diagrams, fermions are represented by straight lines and bosons by wavy lines. In figure 1.1 the process of electron-electron scattering is visualized.

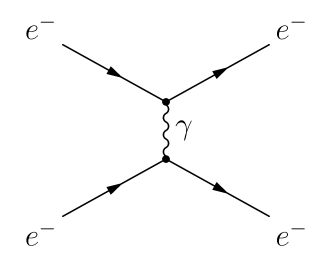

Figure 1.1: Feynman diagram of electron-electron scattering. On the left hand side are the two incoming initial state electrons. They interact via a virtual photon $(\gamma)$ and the scattered final state electrons phase out to the right. The coupling of the electrons to the photon depends on the vertices, shown in the diagram by the dots. 


\subsection{Top Quark Production and Decay}

The top quark is by far the most massive fundamental particle known. Its mass is currently measured at $m_{t}=172.5 \pm 2.3 \mathrm{GeV} / c^{2}[3]$, nearly as heavy as a gold nucleus. Top quarks are most often produced in pairs of one top quark and one antitop quark, while the electroweak production of single top quarks is not yet observed.

At the Tevatron $t \bar{t}$ pairs are produced via quark-antiquark annihilation in $85 \%$ of the cases, while $15 \%$ of the $t \bar{t}$ pairs stem from gluon-gluon fusion. The leading order Feynman diagrams for these two processes are shown in figure 1.2. The current theoretical calculation of the $t \bar{t}$ production cross section at a center of mass energy of $\sqrt{s}=1.96 \mathrm{TeV}$ with a top quark mass of $175 \mathrm{GeV} / c^{2}$ is $6.7 \pm 0.9 \mathrm{pb}[18,19]$.

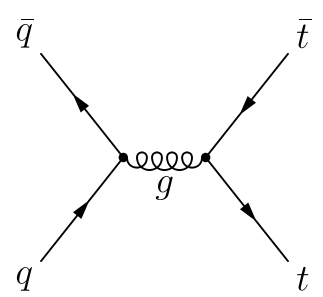

(a)

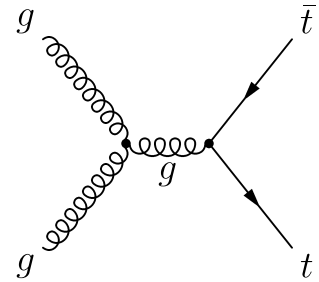

(b)

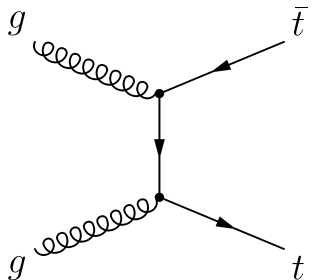

(c)

Figure 1.2: Some leading-order Feynman diagrams of top quark pair production: (a) quarkantiquark annihilation and (b), (c) gluon fusion.

Due to its huge mass compared to the other quarks, the lifetime of the top quark is much shorter than of any other quark. The SM prediction for the top quark lifetime is $0.4 \times 10^{-24} \mathrm{~s}$, which is about 20 times shorter than the timescale for strong interactions. Thus, the top quark decays before it can hadronize, giving the unique opportunity to study a nearly bare quark.

The top quark decays with almost $100 \%$ via $t \rightarrow W^{+} b$ into a $b$ quark and a $W$ boson. The decay rates into other down-type quarks, $t \rightarrow W^{+} d$ and $t \rightarrow W^{+} s$, are suppressed due to the small CKM-Matrix elements of these decays. Since the top quark mass is larger than the sum of the masses of the $W$ boson and the $b$ quark, it decays into a real $W$ boson, while lighter quarks decay into a virtual $W$ boson. The $W$ boson decays into a charged lepton and the corresponding antineutrino or into a quark-antiquark pair. As a result of the universality of the weak interaction the probabilities for the different fermionic decays are equal (disregarding phase space). Due to the three different quark color charges, the decay into quark-antiquark pairs is enhanced by a factor of three, leading to a branching ratio of $1 / 9$ for each of the three leptonic decay modes and branching ratios of $3 / 9$ for decays into a $u \bar{d}$ or $c \bar{s}$ pair. This leads to the following $t \bar{t}$ event topologies: dilepton events in which both $W$ bosons decay leptonically, all-hadronic events in which both $W$ bosons decay hadronically, and lepton+jets events in which one $W$ decays leptonically and the 
other hadronically. The branching ratios for the different top quark decay channels can be found in table 1.3.

In this thesis we use the lepton+jets channel and the analyzed events are thus characterized by a well isolated high-momentum charged lepton and substantial missing transverse energy due to the leptonic $W$ decay, a number of high-energetic hadronic jets due to the hadronic $W$ decay, and by two $b$ jets originating from top quark decay.

\begin{tabular}{|ccc|}
\hline$W_{1}$ decay & $W_{2}$ decay & Branching ratio \\
\hline \hline \multicolumn{3}{|c|}{ Dilepton channel } \\
\hline$e^{+} \nu_{e}$ & $e^{+} \nu_{e}$ & $1 / 81$ \\
$e^{+} \nu_{e}$ & $\mu^{+} \nu_{\mu}$ & $2 / 81$ \\
$e^{+} \nu_{e}$ & $\tau^{+} \nu_{\tau}$ & $2 / 81$ \\
$\mu^{+} \nu_{\mu}$ & $\mu^{+} \nu_{\mu}$ & $1 / 81$ \\
$\mu^{+} \nu_{\mu}$ & $\tau^{+} \nu_{\tau}$ & $2 / 81$ \\
$\tau^{+} \nu_{\tau}$ & $\tau^{+} \nu_{\tau}$ & $1 / 81$ \\
\hline \hline \multicolumn{3}{|c}{ Hadronic channel } \\
\hline \multicolumn{3}{|c}{$\bar{q}$} \\
\hline \hline$e^{+} \nu_{e}$ Lepton + jets channel \\
$\mu^{+} \nu_{\mu}$ & $q \bar{q}$ & $36 / 81$ \\
$\tau^{+} \nu_{\tau}$ & $q \bar{q}$ & $12 / 81$ \\
\hline
\end{tabular}

Table 1.3: Branching ratios for the three different topologies of $t \bar{t}$ events.

\section{$1.3 W$ - Helicity in Top Quark Decays}

In the SM the top quark decays via the charged current weak interaction into a $b$ quark and a $W$ boson. For leptonically decaying $W$ bosons we can immediately write down the Feynman rules for such a vertex, where $\gamma^{\mu}$ denotes the Dirac matrices, $\gamma^{5}$ the chirality operator, and $g$ the coupling constant of the electroweak interaction:

$$
\frac{g}{\sqrt{2}} \gamma^{\mu} \frac{1}{2}\left(1-\gamma^{5}\right)
$$

This expression reflects the vector $\left(\bar{\psi} \gamma^{\mu} \psi\right)$ minus axial vector $\left(\bar{\psi} \gamma^{\mu} \gamma^{5} \psi\right)$ structure, abbreviated $\mathrm{V}-\mathrm{A}$ structure, of the weak interaction in the SM. The corresponding expression for a $\mathrm{V}+\mathrm{A}$ structure, which is not implemented in the $\mathrm{SM}$, is

$$
\frac{g}{\sqrt{2}} \gamma^{\mu} \frac{1}{2}\left(1+\gamma^{5}\right) .
$$


The operator $P_{-}=\frac{1}{2}\left(1-\gamma^{5}\right)$ performs a left handed projection, while $P_{+}=$ $\frac{1}{2}\left(1+\gamma^{5}\right)$ performs a right handed projection. Thus, the $\mathrm{V}-\mathrm{A}$ structure of the SM predicts maximal parity violation, which means, that the weak force only couples to left handed fermions or right handed antifermions.

As mentioned in the previous section, the top quark has a lifetime, that is shorter than the hadronization time. Thus its decay products preserve the helicity content of the underlying weak interaction, giving the opportunity to proof the $\mathrm{V}-\mathrm{A}$ structure of the weak interaction of the SM at high energies. Helicity is defined by the projection of the spin of a particle on the axis, given by the direction of motion $\vec{\sigma} \cdot \hat{p}$. The helicity operator thus projects out two physical states, with the spin along or opposite the direction of motion. If the spin is projected parallel on the direction of motion, the particle is of positive helicity, if the projection is antiparallel to the direction of motion, the particle is of negative helicity. Since in the extreme relativistic limit, the chirality operator is equal to the helicity operator these two helicity modes are also called right handed or left handed, respectively. In cases where the spin is perpendicular to the momentum of the particle, the particle is called longitudinally polarized.

In this thesis, the structure of the weak interaction at high energies is investigated by measuring the helicity fractions of the $W$ boson in top quark decays. In order to discuss which couplings in the $W t b$ vertex could have an impact on the $W$ helicity fractions, the interaction Lagrangian [20] for the most general coupling is considered. The interaction of fermions and gauge bosons can in general be expressed by six form factors with a particular energy scale at which new physics is opened. Assuming the $W$ boson to be on-shell, the number of form factors can be reduced to four:

$$
\begin{aligned}
L & =\frac{g}{\sqrt{2}}\left[W_{\mu}^{-} \bar{b} \gamma^{\mu}\left(f_{1}^{L} P_{-}+f_{1}^{R} P_{+}\right) t-\frac{1}{m_{W}} \partial_{\nu} W_{\mu}^{-} \bar{b} \sigma^{\mu \nu}\left(f_{2}^{L} P_{-}+f_{2}^{R} P_{+}\right) t\right] \\
& +\frac{g}{\sqrt{2}}\left[W_{\mu}^{+} \bar{t} \gamma^{\mu}\left(f_{1}^{L *} P_{-}+f_{1}^{R *} P_{+}\right) b-\frac{1}{m_{W}} \partial_{\nu} W_{\mu}^{+} \bar{t} \sigma^{\mu \nu}\left(f_{2}^{R *} P_{-}+f_{2}^{L *} P_{+}\right) b\right]
\end{aligned}
$$

with

$$
i \sigma^{\mu \nu}=-\frac{1}{2}\left[\gamma^{\mu}, \gamma^{\nu}\right]
$$

The first line describes the decay of a top quark, while the second line is the complex conjugated expression which is denoted by the superscript ${ }^{*}$. $W_{\mu}^{+,-}$is the effective vector field of the $W$ boson while $t$ and $b$ denote the fermion fields of the top and bottom quark, respectively. The $W$ boson mass $m_{W}$ is the energy scale at which the physics beyond the SM becomes apparent. The four form factors are in general complex. Assuming that the interaction of equation 1.5 preserves $\mathrm{CP}$ symmetry the form factors for top quark $\left(f_{1,2}^{L, R}\right)$ and antitop quark $\left(\bar{f}_{1,2}^{L, R}\right)$ decay are taken to be real and have to satisfy the following relations:

$$
f_{1}^{L, R}=\bar{f}_{1}^{L, R}, \quad f_{2}^{L, R}=\bar{f}_{2}^{R, L}
$$


which are already implemented in equation 1.5. At tree level in the SM the formfactor $f_{1}^{L}$ is given by the CKM matrix element $V_{t b}$ and is therefore equal to one, while the other three couplings $f_{1}^{R}$ and $f_{2}^{L, R}$ are all equal to zero, leading to the pure $\mathrm{V}-\mathrm{A}$ structure of the SM.

Though nonvanishing values of the right handed coupling $f_{1}^{R}$ and of the two magnetic couplings $f_{2}^{L, R}$ are not forbidden, several constraints on these anomalous couplings exist. The coupling to right handed $b$ quarks via $f_{1}^{R}$ and $f_{2}^{L}$ is constrained to be less than $0.004[21,22]$ by the $b \rightarrow s \gamma$ branching ratio, which would be increased considerably, if such coupling exists. On the other hand, the decay $b \rightarrow s l^{+} l^{-}$can be sensitive to a left handed $b$ quark coupling like $f_{2}^{R}$, and it imposes a constraint of order 0.03 [22]. However, all these constraints are obtained from indirect measurements assuming that there are no other sources of new physics that could cancel the effects of these couplings on the data [23]. For example the amplitude involving $f_{1}^{R}$ contains the product $V_{t s} \cdot f_{1}^{R}$. The constraint on $f_{1}^{R}$ is based on the value of $V_{t s}=0.04$ assuming $3 \times 3$ CKM unitarity and does not hold if heavier fermions exist.

Under the assumption of a massless $b$ quark which is justified due to the large masses of the $W$ boson and top quark compared to the $b$ quark mass, equation 1.5 leads at tree level to the following decay amplitudes:

$$
\begin{aligned}
\overline{\left|M\left(\lambda_{W}=-\right)\right|^{2}} & =\left|f_{1}^{L}+\frac{m_{t}}{m_{W}} f_{2}^{R}\right|^{2}, \\
\overline{\left|M\left(\lambda_{W}=+\right)\right|^{2}} & =\left|f_{1}^{R}+\frac{m_{t}}{m_{W}} f_{2}^{L}\right|^{2}, \\
\overline{\left|M\left(\lambda_{W}=0\right)\right|^{2}} & =\frac{1}{2}\left|\frac{m_{t}}{m_{W}} f_{1}^{L}+f_{2}^{R}\right|^{2}+\frac{1}{2}\left|\frac{m_{t}}{m_{W}} f_{1}^{R}+f_{2}^{L}\right|^{2} .
\end{aligned}
$$

Here $\lambda_{W}$ denotes the helicity mode of the $W$ boson, " $\lambda_{W}=-"$ means left handed, " $\lambda_{W}=+$ " right handed, and " $\lambda_{W}=0$ " longitudinally polarized.

The fraction $F_{0}$ of longitudinally polarized $W$ bosons strongly depends on the form factors $f_{1}^{L, R}$ and $f_{2}^{L, R}$, making $F_{0}$ a useful observable for measuring these form factors. $F_{0}$ is defined as the ratio of the number of longitudinally polarized $W$ bosons produced with respect to the total number of $W$ bosons produced in top quark decays:

$$
F_{0}=\frac{\Gamma\left(\lambda_{W}=0\right)}{\Gamma\left(\lambda_{W}=0\right)+\Gamma\left(\lambda_{W}=-\right)+\Gamma\left(\lambda_{W}=+\right)}
$$

As mentioned before in the SM the leading behaviour is given by $f_{1}^{L}$, while all other form factors are equal to zero. In this case, the fraction of longitudinally polarized $W$ bosons is given by:

$$
F_{0}=\frac{m_{t}^{2}}{2 m_{W}^{2}+m_{t}^{2}}
$$


Using $m_{W}=80.403 \mathrm{GeV} / c^{2}$ [14] and $m_{t}=172.5 \mathrm{GeV} / c^{2}$ [3], one obtains $F_{0}=0.697$. Under the assumption of a massless $b$ quark the fraction of left handed $W$ bosons is $F_{-}=0.303$, while the right handed fraction $F_{+}$is exactly zero. The production of longitudinally polarized $W$ bosons is enhanced as a consequence of the Goldstone equivalence theorem $[24,25]$. The decay amplitude to longitudinal $W$ bosons is linked to the mechanism of electroweak symmetry breaking and is therefore proportional to the Yukawa coupling. That means that the decay rate into longitudinally polarized $W$ bosons scales with the third power of the top quark mass, while the decay rate to transverse $W$ bosons is governed by the gauge coupling, and therefore increases only linearly with the top quark mass [26].

Next-to-leading order (NLO) corrections to the total decay width and the partial decay widths of the top quark have been performed considering electroweak and finite $W$ width effects as well as QCD-loops and a nonzero $b$ quark mass [27-36]. The corrections amount to about $-10 \%$ for the longitudinal decay width as well as for the total decay width, leading to an only small influence on $F_{0}$ of about one percent. The influence of the nonzero $b$ quark mass on the fraction of right handed $W$ bosons is in the order of one per mille.

Also corrections for possible theories beyond the SM such as supersymmetry (SUSY) or topcolor-assisted technicolor models (TC2) have been performed. In most of the SUSY parameter space the one loop SUSY-QCD corrections and supersymmetric electroweak corrections to the helicity fractions are less than $1 \%$ in magnitude and tend to cancel each other [37]. In technicolor models the corrections to the decay widths can reach under certain conditions the order of the SM NLO corrections [38].

Since $F_{0}$ does not depend on the deviation $\Delta f_{1}^{L}=f_{1}^{L}-1$ from the SM value as long as all other couplings are zero, significant deviations from the SM values for the three fractions $F_{0}, F_{-}$, and $F_{+}$can only arise from the effects of nonzero values of one of the anomalous form factors $f_{2}^{L}$ and $f_{1,2}^{R}$ and therefore indicate new physics.

An additional right handed coupling $f_{1}^{R}$, i.e. a $\mathrm{V}+\mathrm{A}$ current in top quark decay, would lead to a nonzero fraction of right handed $W$ bosons at the expense of $F_{-}$ while $F_{0}$ would remain unchanged. Equation 1.11 and figure 1.3 (a) quantify the effect of such a coupling:

$$
\frac{F_{+}}{F_{-}}=\left(\frac{f_{1}^{R}}{f_{1}^{L}}\right)^{2} .
$$

Assuming that no right handed bottom quark couplings are present, meaning only $f_{1}^{L}$ and $f_{2}^{R}$ take non zero values, the fractions of left handed and longitudinally polarized $W$ bosons are altered, while $F_{+}$remains zero as can be seen in figure $1.3(\mathrm{~b})$. 
A scenario for non zero values of $f_{1}^{L}$ and $f_{2}^{L}$ and vanishing $f_{1,2}^{R}$ is displayed in figure 1.3 (c). In this case all three helicity fractions would be effected.

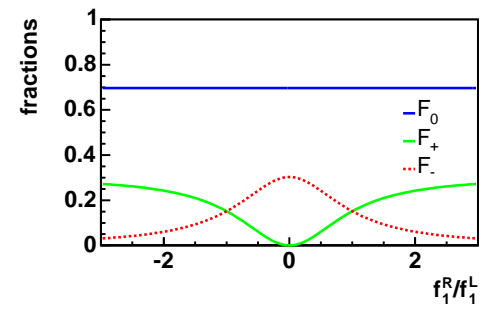

(a)

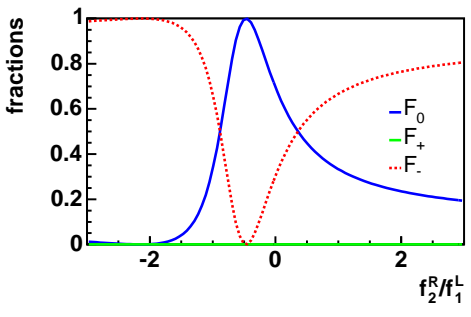

(b)

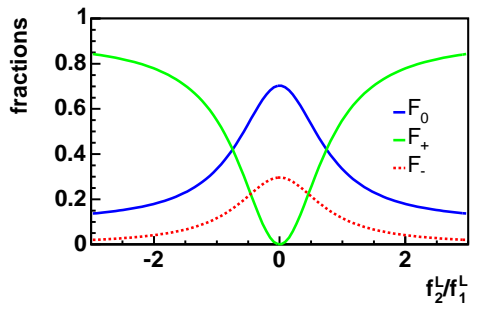

(c)

Figure 1.3: Dependence of the helicity fractions on different anomalous form factor settings for nonzero values of $f_{1}^{L}$ and (a) $f_{1}^{R}$, or (b) $f_{2}^{R}$, or (c) $f_{2}^{L}$.

\subsection{Sensitive Observable $\cos \theta^{*}$}

In the leptonic decay of the $W$ boson the helicity information of the $W$ boson is visible in the decay angle $\theta^{*}$, which is defined as the angle between the momentum of the charged lepton in the $W$ rest frame and the $W$ momentum in the top quark rest frame. Figure 1.4 provides an illustration of this definition. Since the SM considers neutrinos to be massless, there exist only left handed neutrinos, thus the neutrino from the $W$ decay has to be left handed. As a consequence of momentum conservation and spin conservation the charged lepton in the $W$ decay has then always to be right handed. Due to the singly allowed spin configuration of the decay leptons, different distributions of the angle $\theta^{*}$ are obtained for the three helicity modes of the $W$ boson. Figure 1.4 shows the decay of differently polarized $W$ bosons in the $W$ rest frame. Charged leptons from left handed $W$ bosons are likely to fly in the direction of the $b$ quark, leading to large values for $\theta^{*}$ (a), while charged leptons from right handed $W$ bosons fly mostly in opposite direction, leading to small values of $\theta^{*}(\mathrm{c})$. For longitudinally polarized $W$ bosons the $\theta^{*}$ distribution has its maximum at $90^{\circ}$ (b). The comparison between the distributions of the cosine of that decay angle for different helicity modes shows that the observable $\cos \theta^{*}$ is very suitable, in order to differentiate between these modes. For left handed $W$ bosons the $\cos \theta^{*}$ distribution is proportional to $\left(1-\cos \theta^{*}\right)^{2}$, for right handed $W$ bosons proportional to $\left(1+\cos \theta^{*}\right)^{2}$, and for longitudinally polarized $W$ bosons to $\left(1-\cos \theta^{* 2}\right)$. The general $\cos \theta^{*}$ distribution is then given by:

$$
\frac{d N}{d \cos \theta^{*}}=F_{-} \cdot \frac{3}{8}\left(1-\cos \theta^{*}\right)^{2}+F_{0} \cdot \frac{3}{4}\left(1-\cos ^{2} \theta^{*}\right)+F_{+} \cdot \frac{3}{8}\left(1+\cos \theta^{*}\right)^{2} .
$$




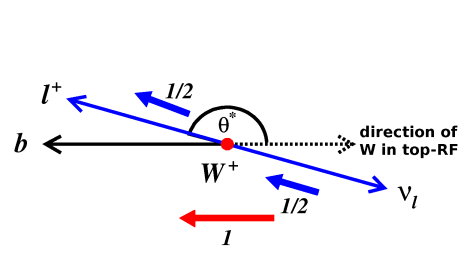

(a)

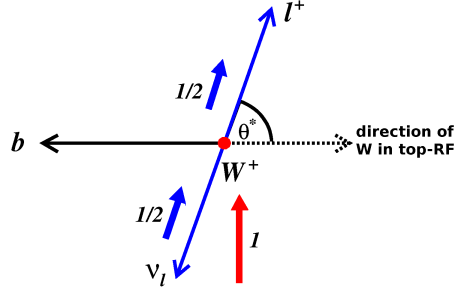

(b)

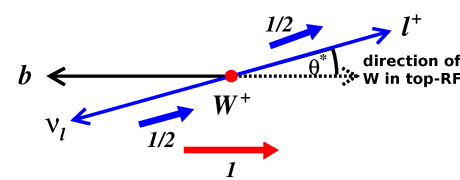

(c)

Figure 1.4: Illustration of the decay of a polarized $W$ boson into a charged lepton and a neutrino in the $W$ boson rest frame. In (a) the $W$ boson is left handed, in (b) the $W$ boson is longitudinally polarized, and in (c) the $W$ boson is right handed. The dotted black arrow represents the direction of the $W$ boson momentum in the rest frame $(\mathrm{RF})$ of the top quark.

The $\cos \theta^{*}$ distributions for the three helicity modes as well as for the SM expectation which is derived by inserting the $\mathrm{SM}$ values for $F_{0}, F_{-}$, and $F_{+}$in equation 1.12 , are presented in figure 1.5.

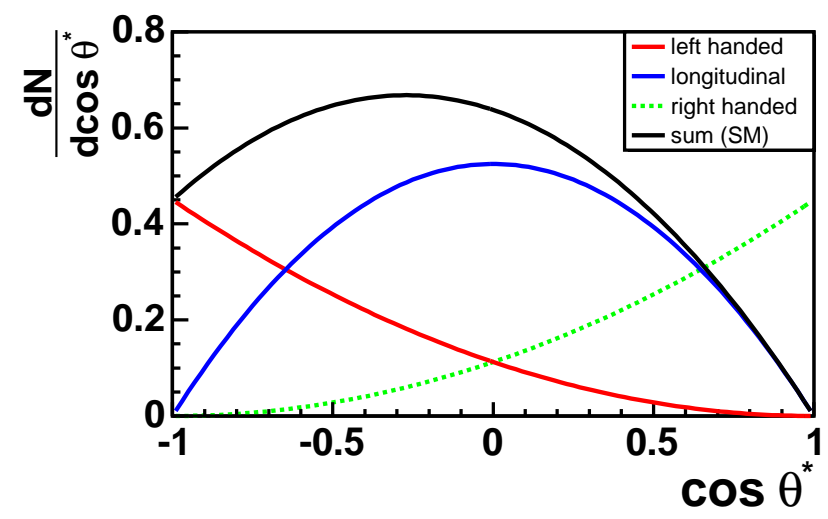

Figure 1.5: Theoretical $\cos \theta^{*}$ distribution for left handed (red), right handed (green dashed) and longitudinally polarized (blue) $W$ bosons. The distribution predicted by the SM is indicated by the black solid line 


\section{Chapter 2}

\section{The CDF II Experiment}

In this chapter we describe the accelerator complex of the Fermi National Accelerator Laboratory (Fermilab) with its different acceleration stages. We also describe the Collider Detector at Fermilab (CDF), one of the two experiments located at the Fermilab, where the data used in our analyses were collected. Particular emphasis is thereby on the main features of the detector that are important for our analysis. Fermilab is located approximately $70 \mathrm{~km}$ west of Chicago in Batavia, Illinois, in the United States of America. More than 2.500 scientists from 34 states and 25 countries partake in the research in elementary particle physics at Fermilab. An aerial shot of Fermilab which covers an area of about $28 \mathrm{~km}^{2}$ is shown in figure 2.1.

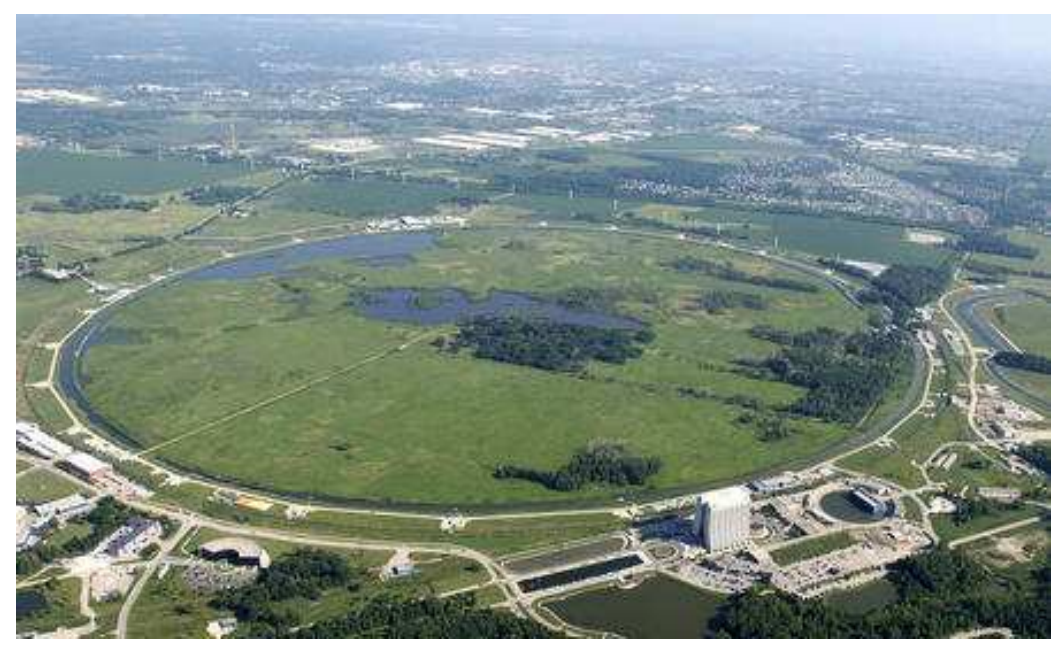

Figure 2.1: Aerial shot of the Tevatron main ring. The CDF site in this view is located at the eight o'clock position direct at the outside of the ring, the $\mathrm{D} \emptyset$ site is at the twelve o'clock position. The Fermilab main building is visible in the foreground.

\subsection{The Collider}

The Fermilab Tevatron Proton-Antiproton Collider is currently the world's highest energy hadron collider until the commissioning of the Large Hadron Collider (LHC) 
at CERN. It operates at a center-of-mass energy of $1960 \mathrm{GeV}$ produced by a proton and an antiproton beam of each $980 \mathrm{GeV}$. In order to reach such high energies, the protons and antiprotons have to run through several acceleration stages. Figure 2.2 gives a schematic overview of the accelerator chain at Fermilab.

\section{FERMILAB'S ACCELERATOR CHAIN}

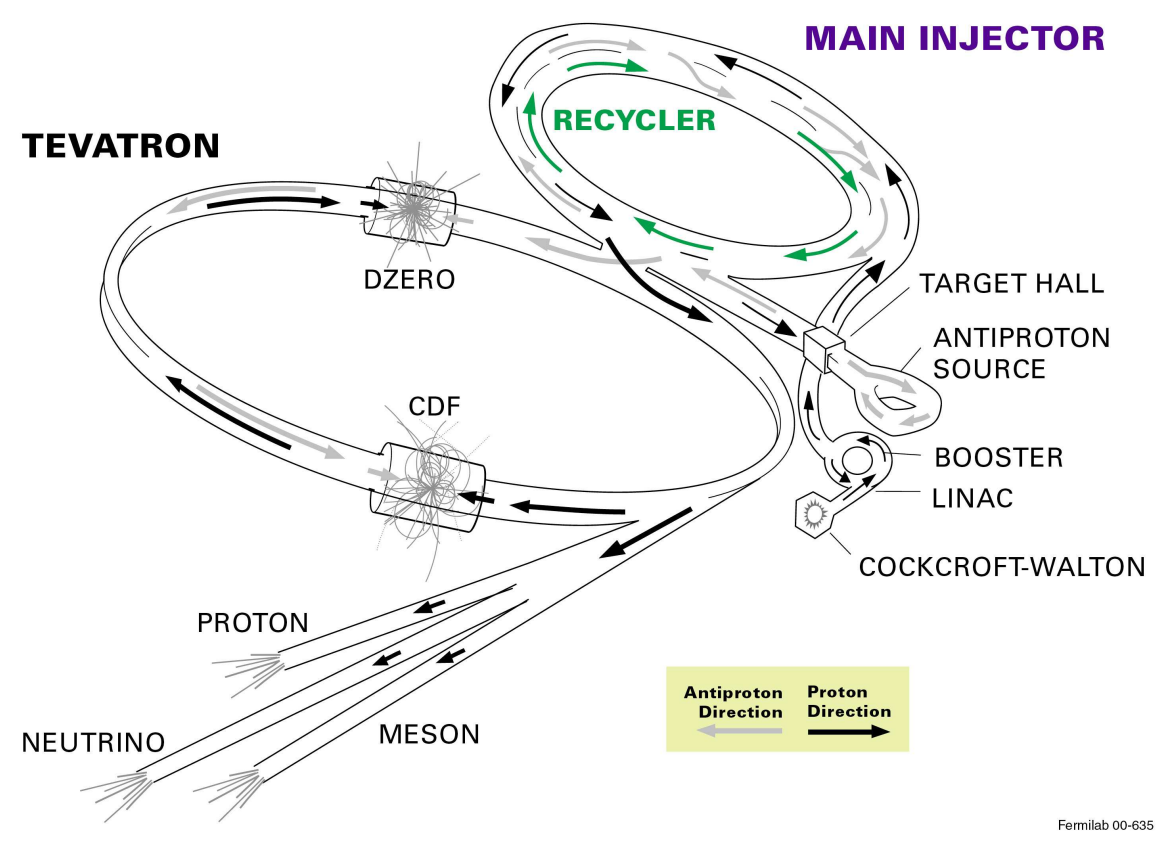

Figure 2.2: Schematic view of Fermilab's accelerator chain for Run II

The first stage on the way to a beam energy of $980 \mathrm{GeV}$ is the Cockcroft-Walton pre-accelerator. Inside this device a Magnetron Ion Source $[41,42]$ produces negative hydrogen ions by surface ionization effects. The Magnetron Source consists of a central cylindrical cathode surrounded by an anode, with hydrogen gas injected into the volume between. The conditions inside the cavity caused by applied electric and magnetic fields create a dense plasma. Protons obtained from this plasma are accelerated towards the cathode and collide with the cathode surface which is coated with Cesium to decrease the work function of the metal. The protons striking the low work function surface capture two electrons and reflect from the surface. A reflected particle normally undergoes resonant neutralization at a very small distance and therefore leaves the surface as a neutral. Due to the low work function of the surface material the energy resonance occurs at much larger distances and therefore the electron transfer to the surface is less probable.

The $H^{-}$ions are extracted and accelerated to $750 \mathrm{keV}$ by a positive voltage in the Cockcroft-Walton pre-accelerator and then sent to the LINear ACcelerator (LINAC). There they are accelerated by oscillating electric fields to $400 \mathrm{MeV}$ over a 
distance of 130 meters. At the end of the LINAC the $H^{-}$ions pass through a carbon foil, where the electrons are stripped off. The generated protons then enter the third stage of acceleration, the booster. In the booster the protons are accelerated from $400 \mathrm{MeV}$ to $8 \mathrm{GeV}$ in $33 \mathrm{~ms}$ and enter the Main Injector where they are accelerated to $120 \mathrm{GeV}$.

Some of the protons are then sent to the antiproton source where they collide with a nickel target. The interaction of the protons with the nickel target produces a wide range of secondary particles including numerous antiprotons. The antiprotons are collected, focused and stored in the Accumulator Ring. When a sufficient number of antiprotons has been produced, they are sent to the Main Injector. There the antiprotons run through the electron-cooling system, which mainly reduces the longitudinal emittance of the beam by mixing the antiprotons with a continuous 4.3 MeV beam of electrons which are provided by a Pelletron accelerator, a type of electrostatic particle accelerator similar to a Van de Graaff generator. The electron beam travels for approximately $20 \mathrm{~m}$ along the same path as the antiprotons and is then sent back to the Pelletron for recirculation. The electrons interact with the antiprotons, cooling the beam and reducing the spread in longitudinal momentum. Antiprotons travelling too fast are slowed down as they bump into electrons, and slow antiprotons are sped up as they are hit by faster electrons. The protons that were not sent to the antiproton source as well as the produced antiprotons are now accelerated in opposite directions up to $150 \mathrm{GeV}$.

The final acceleration stage is the Tevatron, a large collider with a circumference of about six kilometres. Protons and antiprotons are accelerated from $150 \mathrm{GeV}$ to $980 \mathrm{GeV}$ in the Tevatron. Along the Tevatron there are six certain interaction points, called A0, B0, C0, D0, E0, and F0. At two of them the beams are forced to collide with each other, leading to a center-of-mass energy of $\sqrt{s}=1.96 \mathrm{TeV}$. In both collision points experimentalists placed a detector to collect data. The Collider Detector at Fermilab (CDF), where our data come from, is located at the interaction point $\mathrm{B} 0$, while the second detector, the $\mathrm{D} \varnothing$ detector, is located at the interaction point D0. The remaining four interaction points are used for proton injection or beam abort.

An important quantity characterizing colliders is the luminosity $\mathcal{L}$. Luminosity is a measure of particle interactions, specifically the chance that a proton will collide with an antiproton in case of a proton-antiproton collider like the Tevatron. The event rate $n$ for a certain physical process with a cross section $\sigma$ is given by $n=\mathcal{L} \cdot \sigma$. The total number of events $N$ is then obtained by integrating over a certain time interval: $N=\int \mathcal{L} \mathrm{dt} \cdot \sigma$. Figure 2.3 shows the peak luminosity per store, the time interval between two fillings of protons and antiprotons into Tevatron, for Run II. Regions without entries correspond to shut-down periods in which no data were taken. The peak luminosity is reached at the beginning of each store. Due to collisions and beam gas interactions the number of protons and antiprotons stored in the Tevatron decreases during a store leading to an exponential luminosity decrease. 


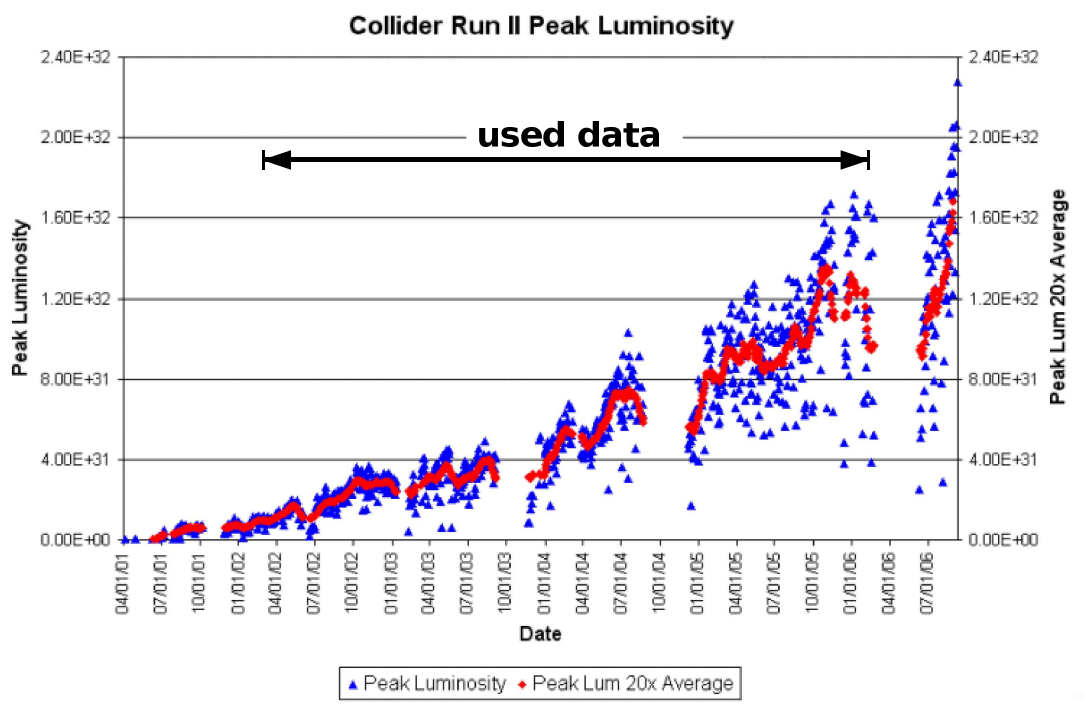

Figure 2.3: Peak luminosity per store since the start of Run II

With the peak luminosity of $2.28 \cdot 10^{32} \mathrm{~cm}^{-2} \mathrm{~s}^{-1}$ achieved in summer 2006 the base line goal for Run II of $1.6 \cdot 10^{32} \mathrm{~cm}^{-2} \mathrm{~s}^{-1}$ has been exceeded and the design goal of $2.7 \cdot 10^{32} \mathrm{~cm}^{-2} \mathrm{~s}^{-1}[40]$ is in range. The base line goal for the integrated luminosity for Run II is $4.4 \mathrm{fb}^{-1}$, the design goal is to collect $8.5 \mathrm{fb}^{-1}$ till the end of 2009 [40]. The increase in the integrated luminosity delivered by the Tevatron and recorded by CDF since the start of Run II is displayed in figure 2.4. The data used in this analysis are taken from March 2002 to February 2006 corresponding to an integrated luminosity of $955 \mathrm{pb}^{-1}$.

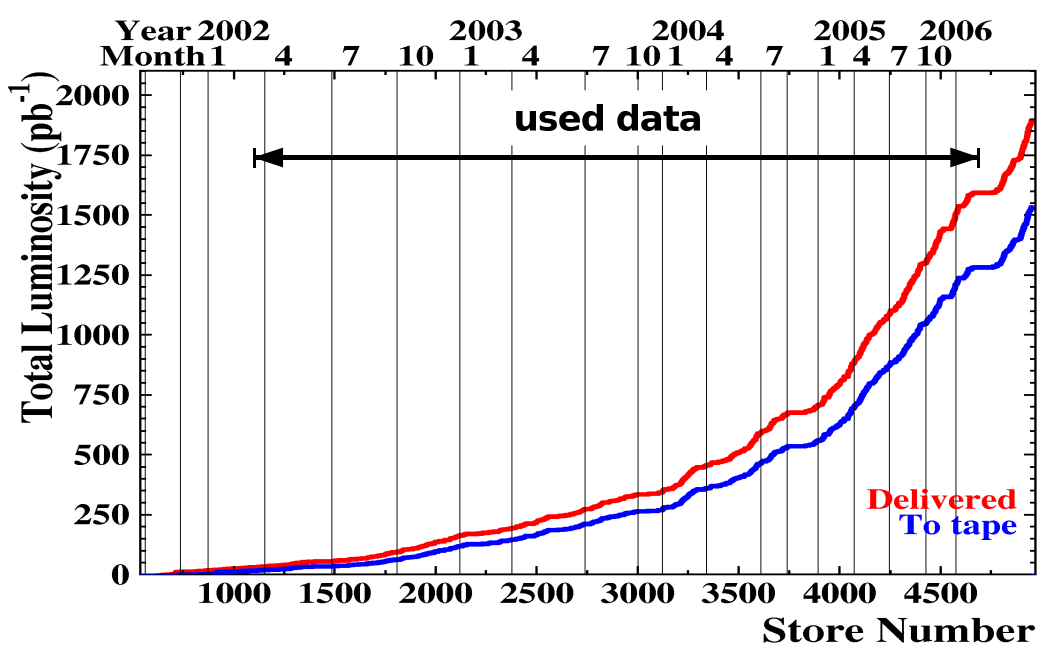

Figure 2.4: Delivered (upper curve) and recorded (lower curve) integrated luminosity for Run II 


\subsection{The CDF II Detector}

The CDF II [43] detector is an azimuthally and forward-backward symmetric general purpose solenoidal detector which combines precision charged particle tracking with fast projective calorimetry and fine grained muon detection. The detector is built and maintained by a collaboration of more than 58 institutes in 13 countries. The Institut für Experimentelle Kernphysik in Karlsruhe is the only German institute in the collaboration. A solid cutaway view of the detector is shown in figure 2.5(a), figure 2.6 shows an elevation view of one half of the detector. Starting from the beam line, the innermost parts of the detector are the tracking systems being contained in a superconducting solenoid with a radius of $1.5 \mathrm{~m}$ and a length of $4.8 \mathrm{~m}$. The solenoid generates a $1.4 \mathrm{~T}$ magnetic field parallel to the beam axis. Calorimetry and muon systems are arranged outside the solenoid. Figure 2.5(b) shows the CDF coordinate system. In that system, $\theta$ and $\phi$ are the polar and azimuthal angles, respectively, defined with respect to the proton beam direction, $z$. The pseudorapidity $\eta$ is defined as $-\ln \left(\tan \frac{\theta}{2}\right)$. The transverse momentum of particles is $p_{\mathrm{T}}=p \cdot \sin \theta$. The main features of the detector systems are explained below in more detail.

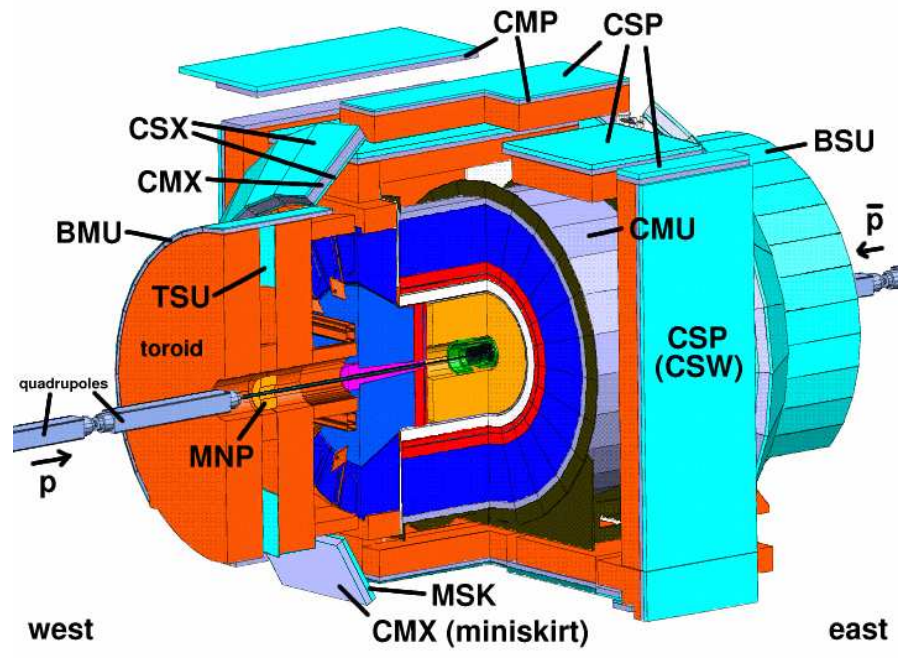

(a)

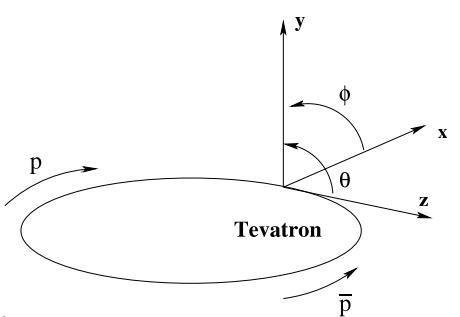

(b)

Figure 2.5: (a) Schematic view of the CDF II detector with its different components. The inner green and orange parts represent the tracking system and the blue ones the calorimeters. The abbreviations refer to the different components of the muon system. (b) The CDF II coordinate system.

\subsubsection{Tracking}

The tracking system consists of the Silicon VerteX detector (SVX II) [44], the Intermediate Silicon Layers (ISL) [45], Layer 00 [46], and of the Central Outer 


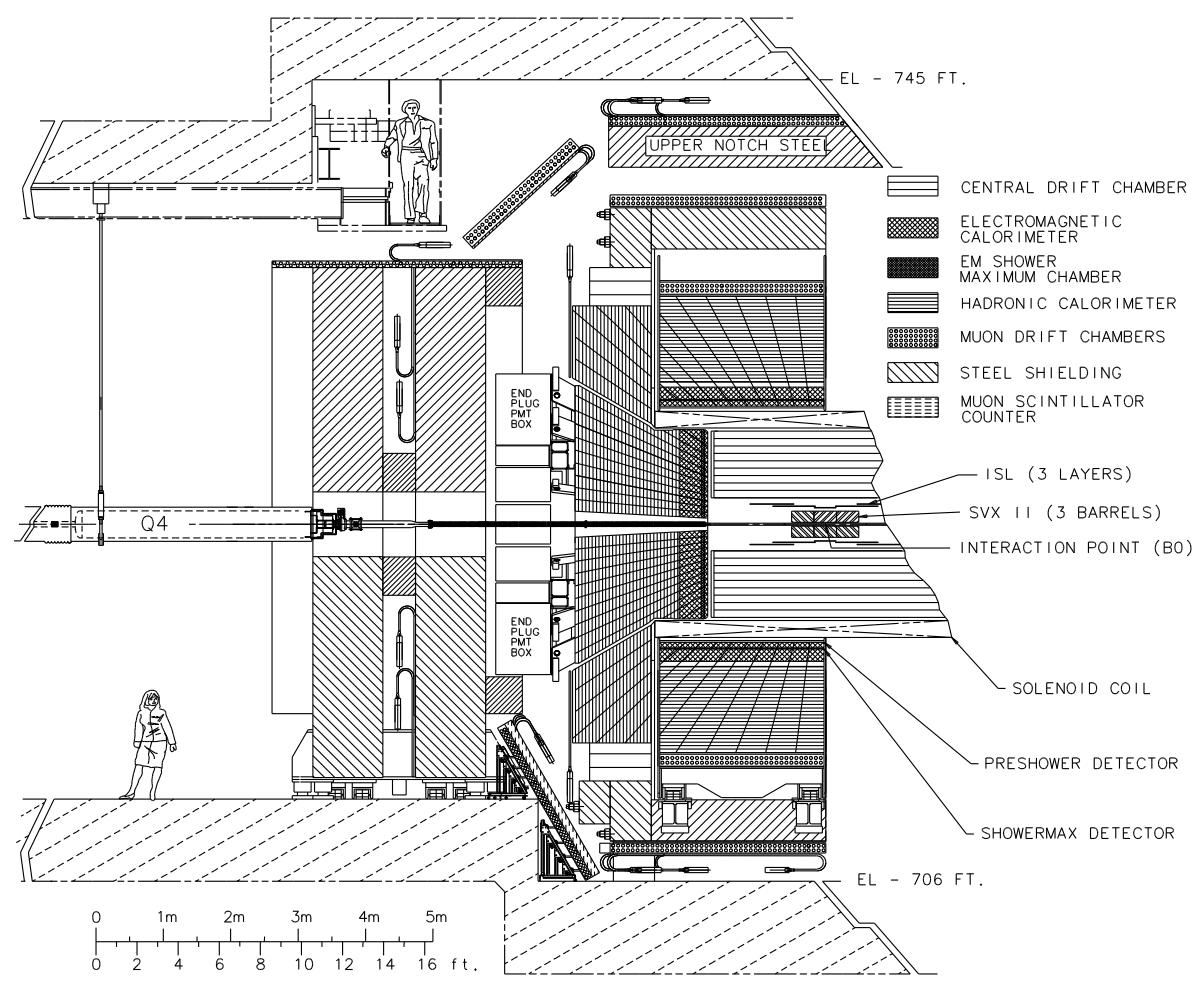

Figure 2.6: Elevation view of one half of the CDF II detector.

Tracker (COT) [47], an open-cell drift chamber surrounding the silicon. A schematic overview of the components is given in figure 2.7. The system of Layer 00, SVX II, and ISL, located immediately outside the beam pipe, provides precise three-dimensional track reconstruction and is used to identify displaced vertices associated with $b$ and $c$ hadron decays. It consists of eight layers in a barrel geometry that extends from a radius of $r=1.5 \mathrm{~cm}$ from the beam line to $r=28 \mathrm{~cm}$. The layer closest to the beam pipe, called Layer 00, is a radiation-hard, single sided detector, while the remaining seven layers are all double-sided detectors. The first five layers after Layer 00 at radii from $2.4 \mathrm{~cm}$ to $10.7 \mathrm{~cm}$ compose the SVX II system and supply $r-\phi$ as well as three $r-z$ and two small angle stereo measurements. The two outer layers at radii of $20 \mathrm{~cm}$ and $28 \mathrm{~cm}$ compose the ISL system. The entire system allows track reconstruction in three dimensions with an impact parameter resolution of $40 \mu \mathrm{m}$. The COT, the main tracking chamber of CDF II, is a $3.1 \mathrm{~m}$ long cylindrical drift chamber segmented into eight concentric superlayers filled with a mixture of about $50 \%$ argon and about $50 \%$ ethane with an admixture of $1.7 \%$ isopropanol and some oxygen to reverse aging effects. In order to handle the high luminosities aimed for in Run II, the COT has been designed to operate with a maximum drift time of $100 \mathrm{~ns}$. Sense wires are arranged in eight alternating axial and $\pm 2{ }^{\circ}$ stereo superlayers with twelve wires each. In an axial superlayer the wires are parallel to the $z$ axis and thus provide only $r-\phi$ information, while in stereo superlayers the wires are arranged with a stereo angle of $\pm 2^{\circ}$ and therefore provide additional $z$ information. The active volume covers the radial range from $40 \mathrm{~cm}$ to $137 \mathrm{~cm}$ and 
CDF Tracking Volume

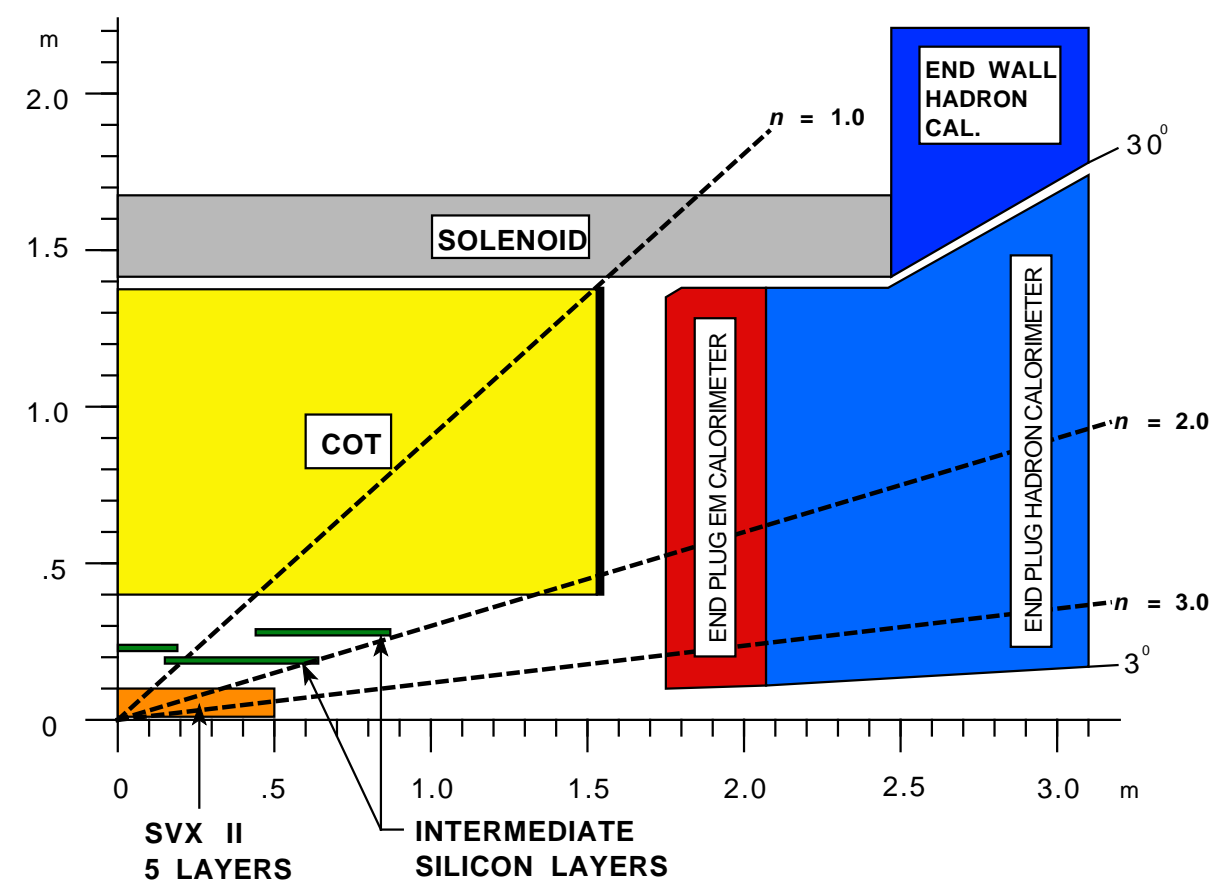

Figure 2.7: Longitudinal view of the CDF II tracking system.

provides coverage for $|\eta| \leq 1$. In the COT the momentum of charged particles is measured precisely with a momentum resolution of $\sigma\left(p_{\mathrm{T}}\right) / p_{\mathrm{T}}^{2}=0.0015(\mathrm{GeV} / c)^{-1}$. In addition, the COT provides also $d E / d x$ information for the measured tracks.

\subsubsection{Calorimetry}

Tracking volume and solenoid are surrounded by the calorimeter system, measuring the energy flow of interacting particles. The calorimeters are arranged in a projective-tower geometry, covering the pseudorapidity region $|\eta| \leq 3.64$. There are five calorimeter systems, two of them in the central region: The Central Electro Magnetic calorimeter (CEM) [48], the Central HAdron calorimeter (CHA) [49], the end-Wall HAdron calorimeters (WHA), the end-Plug Electro Magnetic (PEM), and end-Plug HAdron (PHA) calorimeter [50], covering $2 \pi$ in azimuth and $\eta=-3.64$ to $\eta=3.64$ in pseudorapidity. The central calorimeters cover the pseudorapidity range $|\eta| \leq 1.1$. CEM uses lead sheets interspersed with polystyrene scintillator as active medium and employs phototube readout. Steel absorber interspersed with acrylic scintillator as active medium are used in the CHA. The plug calorimeters cover the pseudorapidity region $1.1 \leq|\eta| \leq 3.64$. They are sampling scintillator calorimeters read out with plastic fibers and phototubes. In table 2.1 the different $|\eta|$ ranges are presented together with the energy resolution for the different calorimeters. 


\begin{tabular}{|l|r|r|}
\hline Calorimeter & $|\eta|$ range & Energy resolution \\
\hline \hline Central Electro Magnetic (CEM) & $|\eta| \leq 1.1$ & $13.5 \% / \sqrt{E} \oplus 2 \%$ \\
end-Plug Electro Magnetic (PEM) & $1.1 \leq|\eta| \leq 3.64$ & $16 \% / \sqrt{E} \oplus 1 \%$ \\
\hline Central HAdron (CHA) & $|\eta| \leq 0.9$ & $75 \% / \sqrt{E} \oplus 3 \%$ \\
end-Wall HAdron (WHA) & $0.9 \leq|\eta| \leq 1.3$ & $75 \% / \sqrt{E} \oplus 3 \%$ \\
end-Plug HAdron (PHA) & $1.3 \leq|\eta| \leq 3.64$ & $74 \% / \sqrt{E} \oplus 4 \%$ \\
\hline
\end{tabular}

Table 2.1: Properties ( $|\eta|$ ranges and energy resolution for single-parton response) of the CDF II calorimeter systems $[48-50]$.

\subsubsection{Muon Reconstruction}

The muon system resides beyond the calorimetry and consists of four subsystems [51]. The Central MUon detector (CMU) consists of four layers of planar drift chambers, detecting muons with transversal momentum larger than $1.4 \mathrm{GeV} / \mathrm{c}$ which penetrate the five absorption lengths of calorimeter steel. The Central Muon uPgrade (CMP) consists of a second set of muon chambers behind an additional $60 \mathrm{~cm}$ of steel in the region $55^{\circ} \leq \theta \leq 90^{\circ}$. The chambers are of fixed length in $z$ and form a box around the central detector. The Central Muon eXtension (CMX) consists of conical sections of drift tubes and scintillation counters located at each end of the central detector and extending in polar angle from $42^{\circ}$ to $55^{\circ}$. At $55^{\circ}$ the CMX system slightly overlaps the coverage provided by the central muon system and extends its pseudorapidity coverage from 0.65 to 1.0. For Run II several new chambers have been added to close gaps in the azimuthal coverage of the CMU, CMP, and CMX. The forward muon system has been replaced by the Intermediate MUon system (IMU) covering a pseudorapidity range of $1.0 \leq|\eta| \leq 1.5$. The location of the subsystems described above can be found in figure 2.5 (a). Table 2.2 gives an overview of some of the properties of the different muon systems.

\begin{tabular}{|l|c|c|c|c|}
\hline & CMU & CMP & CMX & IMU \\
\hline \hline coverage & $|\eta|<0.6$ & $|\eta|<0.6$ & $0.6<|\eta|<1.0$ & $1.0<|\eta|<1.5$ \\
drift tube length & $226 \mathrm{~cm}$ & $640 \mathrm{~cm}$ & $180 \mathrm{~cm}$ & $363 \mathrm{~cm}$ \\
max. drift time & $800 \mathrm{~ns}$ & $1.4 \mu \mathrm{s}$ & $1.4 \mu \mathrm{s}$ & $800 \mathrm{~ns}$ \\
min. muon $p_{\mathrm{T}}$ & $1.4 \mathrm{GeV} / \mathrm{c}$ & $2.2 \mathrm{GeV} / \mathrm{c}$ & $1.4 \mathrm{GeV} / \mathrm{c}$ & $1.4-2.0 \mathrm{GeV} / \mathrm{c}$ \\
\hline
\end{tabular}

Table 2.2: Design parameters of the CDF II muon system

\subsubsection{Trigger System}

The protons and antiprotons are accumulated in bunches separated in space. The maximum crossing rate in the two interaction points B0 and D0 is $7.6 \mathrm{MHz}$ and corresponds to the Tevatron clock cycle of 132 ns. Since only every third bunch is filled with protons or antiprotons, not at all clock cycles a collision occurs. The 
Dataflow of CDF "Deadtimeless"

Trigger and DAQ

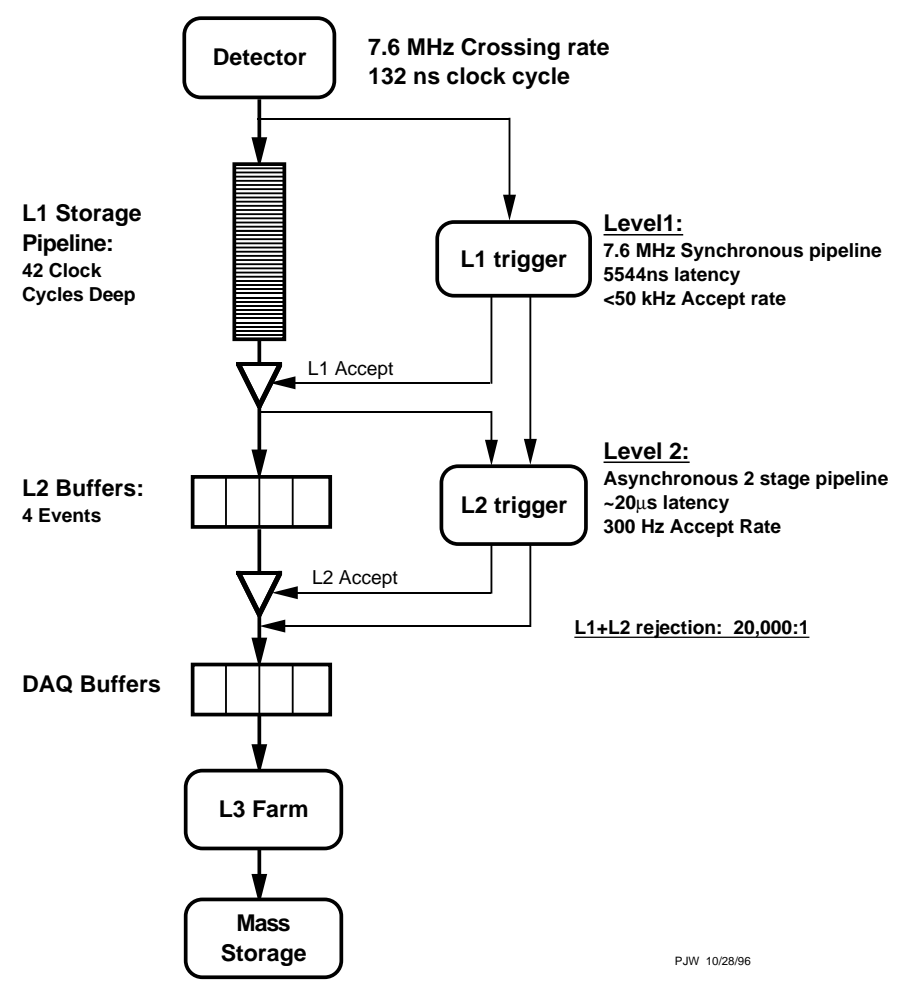

Figure 2.8: Block diagram of the CDF II data flow. Although the mean crossing rate is $1.7 \mathrm{MHz}$, the trigger system must be able to handle the maximum crossing rate of $7.6 \mathrm{MHz}$ corresponding to the Tevatron clock cycle of 132 ns. Therefore in the block diagram the maximum crossing and acceptance rates are indicated.

distance between two filled bunches is 396 ns, resulting in a bunch crossing rate of $2.5 \mathrm{MHz}$. Since the bunches are organized in three so-called trains with twelve bunches each to leave space to abort the beam, the mean crossing rate is reduced to $1.7 \mathrm{MHz}$. However, the amount of information provided by the detector in each collision is impossible to record at such high rates. At present the tape writing speed is limited to an event rate of approximately $75 \mathrm{~Hz}$, and therefore a decision system or trigger system is required to select events of most interest from the large number of minimum bias events. The CDF II trigger system is a three level system with each level providing a sufficient rate reduction for the processing of the next level [52], shown in figure 2.8.

The first two levels are hardware triggers, the third level is a software trigger running on a Linux PC farm. Level-1 uses custom-designed hardware to find physics objects based on a subset of the detector. Three systems run parallel to examine an event: the calorimeter trigger boards to find calorimeter-based objects, the muon trigger cards to identify muons, and the eXtremely Fast Tracker (XFT) which re- 
constructs tracks in the COT and matches these tracks to energy depositions in towers of the calorimeters or hits in the muon chambers. Information from all three systems is used to determine whether an event is passed to Level-2.

The Level-2 trigger performs a limited event reconstruction utilizing a customdesigned hardware consisting of several asynchronous subsystems, e.g. the hardware cluster finder using calorimeter information. In addition, data from the CEntral Shower maximum detector (CES) can be used to improve the identification of electrons and photons. The Silicon Vertex Tracker (SVT) [53] allows to select tracks with large impact parameter. Approximately 300 events are accepted per second by Level-2 and transferred to the Level-3 processor farm [54].

The Level-3 trigger is a processor-based filtering mechanism which has access to the full event record. The Level-3 trigger decision is made based on the particle content and event topology. Accepted events are then written to permanent storage with approximately $75 \mathrm{~Hz}$ at present. To facilitate the handling of the huge data volumes collected, events passing all three trigger levels are split into eight different streams. To which stream a certain event belongs depends on the triggers an event has passed, e.g. all events passing any of the highly energetic lepton triggers end up in "stream B". 


\section{Chapter 3}

\section{Event Selection and Background Estimation}

In this chapter the used data and Monte Carlo samples are briefly described. Also a short introduction into the different Monte Carlo generators that were used is given. We then describe the selection of $t \bar{t}$ candidate events which follows the suggestion of the CDF lepton+jets group, give an overview of the different background sources one has to handle, and present the estimation of the background fraction in the data.

\subsection{Data Samples}

For this analysis the data taken from March 2002 to February 2006 are used. Since we focus on the lepton + jets channel the data stream including all inclusive high $-p_{\mathrm{T}}$ lepton samples recorded in this period is analyzed. Data are reprocessed offline before being analyzed by the different physics groups. During the reprocessing calibrations used online are checked and corrected, silicon alignment is corrected, tracks are refit, cluster energies are checked, and leptons are identified. Also special algorithms are run which identify jets and search for secondary vertices. The data taken from March 2002 to August 2004 were reprocessed with CDFSOFT2 [55] version 5.3.3 and stripped into two datasets, bhel0d and bhmu0d, while data taken from December 2004 to February 2006 were reprocessed with CDFSOFT2 version 6.1.2 and stripped into the datasets bhel0h, bhmu0h, bhel0i, and bhmu0i. The electron data, the bhelXX samples, have to pass the high- $p_{\mathrm{T}}$ central electron Level-3 trigger ELECTRON_CENTRAL_ET18, while the muon data, the bhmuXX samples, have to pass one of the high- $p_{\mathrm{T}}$ central muon Level-3 triggers MUON_CMUP18 or MUON_CMX18. The main requirements of these triggers are tracks in the COT and a matching signal in the corresponding detector component: CEM for the electrons, CMU and CMP for the CMUP muons, and CMX for the CMX muons.

As mentioned in the chapter before, the time interval between two fillings of protons and antiprotons into the Tevatron is called a store. Every store consists of several temporal sections, called runs, which begin with online data taking and end 
when a detector subsystem fails, making full data collection temporarily impossible. All runs are examined both online and offline to verify the quality of data recorded. This is done for all subsystems separately. Only runs in which certain detector components were active and determined to be "good" enter the goodrun list [56] and are used for physics analyses. Dependent on the demanded components there are different goodrun lists. For our analysis we require a functional silicon detector and COT, a functional calorimeter system, and a functional muon system and therefore use the goodrun list version 13 of the top quark group [57]. The total dataset used in this analysis corresponds to an integrated luminosity of $\mathcal{L}=955 \pm 57 \mathrm{pb}^{-1}$ for CEM/CMUP and $\mathcal{L}=941 \pm 56 \mathrm{pb}^{-1}$ for CMX. Table 3.1 shows the six data sets together with the corresponding run ranges, date of data taking, and number of events contained in each sample. In addition we also provide the integrated luminosity of each sample. In case of the muon sample bhmu0d two values for the integrated luminosity are given, the first value corresponds to the CMUP muons, the second to the CMX muons. The difference is due to a not fully functional CMX detector in the beginning of Run II data taking.

\begin{tabular}{|c|l|c|c|c|}
\hline Sample & Run range & Date & Events & $\mathcal{L}\left[\mathrm{pb}^{-1}\right]$ \\
\hline \hline bhel0d $(\mathrm{e})$ & $141544-186598$ & $03 / 23 / 2002-08 / 22 / 2004$ & 1255715 & 333 \\
bhmu0d $(\mu)$ & $141544-186598$ & $03 / 23 / 2002-08 / 22 / 2004$ & 552401 & $333 / 319$ \\
\hline bhel0h $(\mathrm{e})$ & $190697-203799$ & $12 / 07 / 2004-09 / 04 / 2005$ & 1176549 & 363 \\
bhmu0h $(\mu)$ & $190697-203799$ & $12 / 07 / 2004-09 / 04 / 2005$ & 574704 & 363 \\
\hline bhel0i $(\mathrm{e})$ & $203819-212133$ & $09 / 05 / 2005-02 / 22 / 2006$ & 730697 & 258 \\
bhmu0i $(\mu)$ & $203819-212133$ & $09 / 05 / 2005-02 / 22 / 2006$ & 358639 & 258 \\
\hline
\end{tabular}

Table 3.1: Used data samples with run range, date of data taking, number of events contained in each sample, and integrated luminosity of each sample (the two values for the bhmu0d sample correspond to CMUP/CMX).

\subsection{Monte Carlo Samples}

To accomplish this analysis, we have to determine efficiencies and resolutions, estimate background rates and systematic uncertainties, and perform checks of the developed method. For this purpose we make use of samples of simulated events. According to the setting in the event generator it is possible to generate different kinds of events. Besides from signal and background simulation corresponding to the theoretical predictions of the SM also samples corresponding to non-SM theories can be generated. The next sections describe three different event generators and the different samples of generated events used in our analysis. 


\subsubsection{Monte Carlo Generators}

Physics event generators based on the Monte Carlo (MC) method randomly generate hard parton interactions according to the probability density of phase space and the matrix element of certain physical processes. Besides the elementary hard subprocess, i.e. the interaction of two incoming beam particles or their constituents producing one or more primary outgoing fundamental particles, also initial and final state radiation, the decay of heavy objects, and the hadronization of the partons produced in the interaction are simulated. Since this hadronization process takes place at a low momentum transfer scale, for which the strong coupling is large, perturbation theory is not applicable. In the absence of a firm theoretical understanding of nonperturbative processes, it must be described by a phenomenological model, which can be different for the various generators. In the following three different $\mathrm{MC}$ event generators used in this analysis are briefly described.

\section{Pythia}

PYThia [58] is a program for the generation of high-energy physics events, i.e. for the description of collisions at high energies between elementary particles such as $e^{+}, e^{-}, p$, and $\bar{p}$ in various combinations. PYTHIA contains theory and models for a number of physics aspects, including hard and soft interactions, parton distributions, initial and final state parton showers, multiple interactions, fragmentation and decay. For the treatment of the hadronization process the Lund string model is implemented. In this model color flux tubes are stretched between final-state quarks and antiquarks. The potential energy stored in these strings can be converted into new quark-antiquark pairs, which then build-up colorless hadrons. The program is largely based on original research, but also borrows many formulae and other knowledge from the literature.

\section{Herwig}

HERWIG [59] is a general-purpose event generator for high energy hadronic processes which includes the simulation of hard lepton-lepton, lepton-hadron and hadronhadron scattering and soft hadron-hadron collisions in one package. Particular emphasis is thereby on the detailed simulation of QCD parton showers. Therefore HERWIG uses the parton-shower approach for initial state and final state QCD radiation, including color coherence effects and azimuthal correlations both within and between jets. Other special features of HERWIG are QCD jet evolution with soft gluon interference considered via angular ordering, a cluster model for jet hadronization via non-perturbative gluon splitting, and a similar cluster model for soft and underlying hadronic events. The main difference between HERWIG and PYTHIA is the different modelling of the hadronization process. While PYTHIA uses the Lund string model, HERWIG makes use of a cluster model. 


\section{Alpgen}

ALPGEN [60] is an event generator, dedicated to the study of multiparton hard processes in hadronic collisions. The ALPGEN code calculates the exact matrix elements for a large set of QCD and electroweak parton-level processes at the leading order perturbation theory. Parton-level events are generated and full information on their color and flavor structure is provided, enabling the evolution of the partons into fully hadronized final states. The large energies available at the Tevatron make final states with several hard and well separated jets a rather common phenomenon. These multijet final states can originate directly from hard QCD radiative processes, or from the decay of massive particles, such as $W$ or $Z$ bosons. Among other final states, the current version of ALPGEN describes $W$ boson production in association with heavy quark and light quark production, and is therefore well suited to model parts of the background occurring in our analysis.

\subsubsection{Used MC Samples}

For every generated event the resulting particles are handed to the detector simulation of the CDF II detector. The detector response to these particles is modeled based on a detailed simulation with the GEANT3 package [61] which describes the passage of particles through matter. In order to get realistic simulated events that can be compared with data, the MC samples are simulated with run dependent settings. Once processed by GEANT the simulated events can be treated in the same way as real data which has been collected by the detector.

For our analysis we need several Monte Carlo samples with different settings. The method to extract the $W$ boson helicity fractions from the $\cos \theta^{*}$ distribution requires samples describing signal as well as samples describing background events. Our $t \bar{t}$ signal sample consists of about 3.5 million events generated with PYTHIA. Here a top quark mass of $m_{t}=175 \mathrm{GeV} / \mathrm{c}^{2}$ was used. One part of the background we have to deal with results from $W$ boson production in association with heavy quark and light quark production. In order to model this particular background, MC samples are generated with ALPGEN and forwarded to HERWIG which models the hadronization.

For validation of our method we use samples generated with a customized version of the HERWIG program (GGWIG samples) in which the helicity of one $W$ is fixed to be left handed, right handed or longitudinally polarized. The helicity of the second $W$ boson from the $t \bar{t}$ pair is taken according to the Standard Model.

For systematic studies several $t \bar{t}$ signal Monte Carlo samples with different settings, such as different top quark masses, more or less Initial and Final State Radiation (ISR, FSR) with respect to the default $t \bar{t}$ signal sample, or different Parton Distribution Functions (PDF), are used. Table 3.2 gives a summary of the utilized Monte Carlo samples together with the simulated physical process, the name of 


\begin{tabular}{|c|c|c|c|c|}
\hline Sample & Process & Run Range/Comment & Generator & Events \\
\hline \multicolumn{5}{|c|}{ Signal samples } \\
\hline ttopkl & $t \bar{t}, m_{t}=175 \mathrm{GeV} / c^{2}$ & $141544-179056$ & PYTHIA & $2.0 \mathrm{M}$ \\
\hline ttopyl & $t \bar{t}, m_{t}=175 \mathrm{GeV} / c^{2}$ & $182843-183079$ & PYThiA & $0.1 \mathrm{M}$ \\
\hline ttopvl & $t \bar{t}, m_{t}=175 \mathrm{GeV} / c^{2}$ & $191208-201155$ & PYThiA & $1.0 \mathrm{M}$ \\
\hline ttoptl & $t \bar{t}, m_{t}=175 \mathrm{GeV} / c^{2}$ & $201212-203799$ & PYтніА & $0.4 \mathrm{M}$ \\
\hline \multicolumn{5}{|c|}{ Background samples } \\
\hline ltop4n & $W(\rightarrow e \nu)+4 p$ & & Alpgen + Herwig & $0.5 \mathrm{M}$ \\
\hline ltop4m & $W(\rightarrow \mu \nu)+4 p$ & & Alpgen + Herwig & $0.5 \mathrm{M}$ \\
\hline ltop $2 b$ & $W(\rightarrow e \nu)+2 b+2 p$ & & Alpgen + Herwig & $0.5 \mathrm{M}$ \\
\hline ltop $5 \mathrm{~b}$ & $W(\rightarrow \mu \nu)+2 b+2 p$ & & Alpgen + Herwig & $0.5 \mathrm{M}$ \\
\hline \multicolumn{5}{|c|}{ Systematic samples } \\
\hline ttopel & $t \bar{t}, m_{t}=178 \mathrm{GeV} / c^{2}$ & & PYTHIA & $1.2 \mathrm{M}$ \\
\hline ttopvh & $t \bar{t}, m_{t}=175 \mathrm{GeV} / c^{2}$ & & Herwig & $0.2 \mathrm{M}$ \\
\hline ttopbr & $t \bar{t}, m_{t}=178 \mathrm{GeV} / c^{2}$ & less ISR & PYThiA & $1.0 \mathrm{M}$ \\
\hline ttopdr & $t \bar{t}, m_{t}=178 \mathrm{GeV} / c^{2}$ & more ISR & PYTHIA & $1.0 \mathrm{M}$ \\
\hline ttopfr & $t \bar{t}, m_{t}=178 \mathrm{GeV} / c^{2}$ & less FSR & PYThiA & $1.0 \mathrm{M}$ \\
\hline ttopkr & $t \bar{t}, m_{t}=178 \mathrm{GeV} / c^{2}$ & more FSR & PYTHIA & $0.5 \mathrm{M}$ \\
\hline ttopir & $t \bar{t}, m_{t}=178 \mathrm{GeV} / c^{2}$ & MRST72 & PYThiA & $1.0 \mathrm{M}$ \\
\hline ttopjr & $t \bar{t}, m_{t}=178 \mathrm{GeV} / c^{2}$ & MRST75 & PYTHIA & $1.0 \mathrm{M}$ \\
\hline \multicolumn{5}{|c|}{ Polarization samples } \\
\hline utop0i & $t \bar{t}, m_{t}=175 \mathrm{GeV} / c^{2}$ & $W^{+}$left handed & Herwig & $0.1 \mathrm{M}$ \\
\hline utop1i & $t \bar{t}, m_{t}=175 \mathrm{GeV} / c^{2}$ & $W^{+}$right handed & Herwig & $0.1 \mathrm{M}$ \\
\hline utop2i & $t \bar{t}, m_{t}=175 \mathrm{GeV} / c^{2}$ & $W^{+}$longitudinal & Herwig & $0.1 \mathrm{M}$ \\
\hline
\end{tabular}

Table 3.2: Used Monte Carlo samples for signal and background modelling as well as for systematic studies and consistency checks of our analysis method. For information the underlying physical process is given together with the top quark mass, where possible. For the signal samples the run range they correspond to is also provided, while for the systematic samples we mention the setting that differs from that of the signal samples. In addition, we give the generator the samples were generated with and the number of generated events. 
the program the samples were generated with, special settings, and the number of generated events.

\subsection{Event Selection}

In this thesis we focus on the lepton+jets channel for several reasons. The branching fraction is comparable to that one of the all-hadronic channel and larger than the branching fraction of the dilepton channel, as can be seen in section 1.2. The background fractions are considerably higher than in the dilepton channel, but still manageable. Due to the fewer number of jets in the dilepton channel compared to other channels the combinatorics, one has to deal with during the reconstruction of the $t \bar{t}$ pair, are much smaller than in any other channel. But the crucial disadvantage of the dilepton channel are the two produced neutrinos which appear only in the form of missing transverse energy in the detector. Individual reconstruction of the two neutrinos is thus not possible. In the all-hadronic channel at least six jets have to be combined to two top quarks, leading to a huge number of different event interpretations. In this respect the lepton+jets channel is a compromise with a large number of events and manageable background and combinatorics. The selection of $t \bar{t}$ candidates in this channel follows the suggestion of the lepton+jets group for the CDF software version 5.3.3 [62].

\section{Event Topology}

In the analyzed channel, one top quark decays semileptonically and the second top quark decays hadronically, leading to a signature of one isolated charged lepton, missing transverse energy, and at least four jets.

\section{Lepton Requirements}

We require the charged lepton to be an isolated tight electron or muon candidate. Electromagnetic objects have to fulfill several requirements to be identified as an electron or muon, respectively. Dependent on the calorimeter, the electron candidates were detected in, we distinguish between the central electrons (detected in the CEM) and the forward electrons, also called plug or phoenix electrons (detected in the PHX). In case of a muon candidate we distinguish between CMX muons, found in the CMX muon chamber, and CMUP muons which have to be detected in the CMU as well as in the CMP muon chamber.

A lepton candidate is considered isolated if the non-lepton $E_{\mathrm{T}}$ in a cone in the $\eta-\phi$ plane of radius 0.4 centered around the lepton is less than $10 \%$ of the lepton $E_{\mathrm{T}}$ or $p_{\mathrm{T}}$, respectively. The transverse energy of an electron candidate is required to be $E_{\mathrm{T}}>20.0 \mathrm{GeV}$. For muon candidates the energy cannot be measured in the detector, since muons are not fully absorbed in the muon chambers. The energy of a muon is thus measured from the transverse momentum of the corresponding track and therefore we require $p_{\mathrm{T}}>20.0 \mathrm{GeV} / \mathrm{c}$. There are several other requirements 
on the lateral shower profile $L_{\text {shr }}$ in the calorimeter, on the ratio of the energy deposited in the hadronic calorimeter with respect to the electromagnetic calorimeter energy $E_{\text {had }} / E_{\text {em }}$, and on other observables. A summary of all lepton requirements can be found elsewhere $[63,64]$.

CMUP, CMX, CEM, and PHX leptons that fulfill all these requirements are called "tight" leptons. For our analysis only events containing tight CEM, CMX, or CMUP leptons are considered, but plug electrons are used for the dilepton veto.

Beyond these lepton requirements there are several other requirements the entire event has to fulfill. Electron events are rejected if the electron stems from a conversion of a photon into an $e^{+} e^{-}$pair. Also cosmic muon events are removed. The main cuts are now described in more detail.

\section{Dilepton veto}

To ensure, that we have only one charged lepton in the event, the dilepton veto is applied, which means that events with a primary tight lepton and an additional tight or loose lepton are rejected.

Besides the tight lepton categories there are several other categories of "loose" leptons. Either they are not detected in one of the detector parts mentioned above, e.g. a muon only found in the CMU but not in the CMP is considered "loose", or they fulfill not all requirements for tight leptons. For example all non-isolated lepton candidates that pass all other selection cuts are considered "loose".

\section{$z$ Vertex Cut}

The event $z$ vertex is the vertex on the $z$ axis closest to the maximum- $E_{\mathrm{T}}$ tight lepton with at least two good COT tracks. It is used to cluster jets and serves as seed for the determination of the primary vertex of the event. In order to ensure that the high-energy lepton comes from the event $z$ vertex, events for which the lepton $z_{0}$ which is the intersection of the lepton momentum with the beam axis is more than $5.0 \mathrm{~cm}$ away from the $z$ vertex are rejected.

\section{$Z$ Boson Veto}

In order to get a further reduction of $Z$ boson events, we remove events in which the tight lepton and a second object form an invariant mass within a window of the $Z$ mass $\left(76 \mathrm{GeV} / c^{2} \leq M \leq 106 \mathrm{GeV} / c^{2}\right)$. If the tight lepton is an electron, the second object may be an isolated electromagnetic object, a reclustered jet with electromagnetic fraction greater than 0.95 , or an opposite-signed isolated track. If the tight lepton is a muon, the second object may be an isolated muon or an opposite-signed isolated track. 


\section{Jet Reconstruction}

As a consequence of quark confinement single final state quarks are forced to form colorless hadrons. The particles produced in this hadronization process form particle jets which are measured as calorimeter jets in the electromagnetic and hadronic calorimeters. Calorimeter jets are identified and reconstructed using the cone algorithm JETCLU [65] which sums calorimeter energy in a cone of radius 0.4 in $\eta$ - $\phi$ coordinates. Calorimeter towers originating from any tight isolated lepton are removed, before clustering the jets. Due to several calorimeter and physics effects the energy of the jets has to be corrected. The corrections are divided into different levels to adapt the needs of different steps of physical analyses. For the event selection we correct all jets up to level 4 . That implies $\eta$-dependent jet energy correction to make the calorimeter response to jet energies uniform in $\eta$ and correction for multiple interactions whose contribution to the cluster energy of a jet must be subtracted. The transverse energy of a jet, corrected in such way, has to fulfill $E_{T}>15 \mathrm{GeV}$ and the pseudorapidity of the jet is required to be $|\eta|<2.0$. Only events with at least four jets are selected for our analysis.

For the complete reconstruction of the top quarks further corrections on the jet energies have to be applied. To derive the energy of the underlying parton we correct jets with level 7 . In addition to the corrections corresponding to level 4 the jets are adjusted for any non-linearity and energy loss in the un-instrumented regions of the central calorimeter (level 5). Also the underlying event energy is subtracted from the jet energy (level 6). The final stage in the level 7 correction are out-of-cone corrections which adjust the energy of the particle-jet obtained by level 6 correction for radiation outside the clustering cone, resulting in the energy of the parton from which the jet originates. More information on the jet corrections can be found elsewhere [66].

\section{Missing Transverse Energy}

Neutrinos do not interact with any of the detector parts. Consequently, in events in which one or more neutrinos are produced there has to be missing transverse energy. Missing transverse energy $\left(\vec{E}_{\mathrm{T}}\right)$ is defined by

$$
\overrightarrow{\mathbb{E}_{\mathrm{T}}}=-\sum_{i} E_{\mathrm{T}}^{i} \hat{n}_{i}, i=\text { calorimeter tower number with }|\eta|<3.6
$$

where $\hat{n}_{i}$ is a unit vector perpendicular to the beam axis and pointing at the $\mathrm{i}^{\text {th }}$ calorimeter tower. We also define the magnitude of this vector $\mathbb{E}_{\mathrm{T}}=\left|\vec{E}_{\mathrm{T}}\right|$. Because this calculation is based on calorimeter towers, $\mathbb{E}_{\mathrm{T}}$ has to be adjusted for the effect of the jet corrections for all jets with $E_{\mathrm{T}}>8 \mathrm{GeV}$ and $|\eta|<2$.4. For the event selection level 4 corrections are applied. For the complete event reconstruction we correct $\mathbb{E}_{\mathrm{T}}$ only up to level 6 instead of level 7 to avoid double counting of the out-of-cone energy. In muon events, the transverse momentum $p_{\mathrm{T}}$ of the muon is added to the sum and a correction is applied to remove the average ion- 
ization energy released by the muon in traversing the calorimeter. For our analysis the corrected missing transverse energy in an event is required to be at least $20 \mathrm{GeV}$.

\section{$b$ tagging}

Because in $t \bar{t}$ events two $b$ jets should exist, we require, that at least one jet is tagged by the SecVtx [67] algorithm as $b$ jet. The SecVtx algorithm utilizes the fact, that $b$ hadrons, i.e. hadrons containing a $b$ quark, have relatively long lifetimes, on average $1.5 \mathrm{ps}$, leading to an observable traveled distance before decaying. At the Tevatron the transverse momentum of the $b$ quarks in $t \bar{t}$ events is on average $65 \mathrm{GeV} / c$ and therefore the $b$ hadrons travel on average a distance of $7.5 \mathrm{~mm}$ from the primary interaction point before they decay. Thus, the existence of displaced vertices in an event is evidence for $b$ quarks. The SecVtx algorithm looks for tracks in the cone of each jet, that do not match to the primary interaction vertex, but intersect in a secondary, displaced vertex. Only tracks with a minimum number of hits in the silicon detectors and an impact parameter $d_{0}$ smaller than $0.3 \mathrm{~cm}$ are considered "good" and therefore taken into account. Since at least two tracks are needed to reconstruct an intersection, only events containing jets with at least two "good" tracks are considered "taggable". Once a secondary vertex is found, the distance between the two vertices in the $r-\phi$ plane, $L_{x y}$, is calculated. To get tagged as a $b$ jet, the significance $L_{x y} / \sigma_{x y}$ of the displacement has to be equal or larger than 3.0. Figure 3.1 gives an schematic view of a secondary vertex with the impact parameter $d_{0}$ and the distance between both vertices $L_{x y}$ which is equal to the two dimensional projection of the decay length of the $b$ hadron.

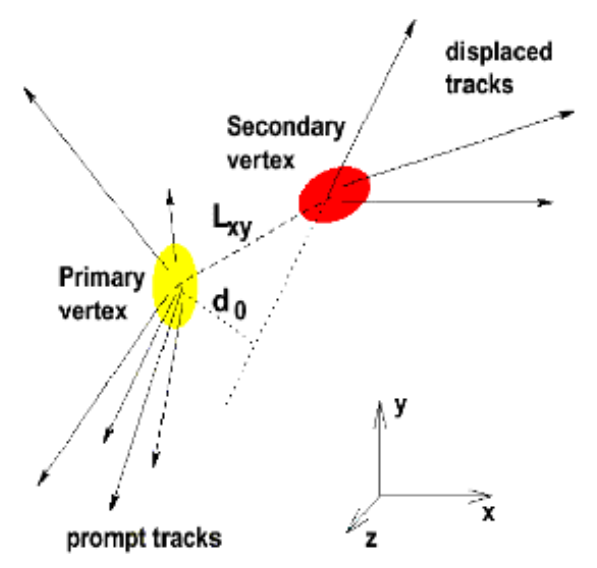

Figure 3.1: Schematic view of a secondary vertex with impact parameter $d_{0}$ and decay length $L_{x y}$.

\section{Summary}

A typical cut flow for a certain sample can be found in table 3.3 for the $t \bar{t}$ signal Monte Carlo sample ttopkl generated with PYTHIA. After applying the goodrun list 


\begin{tabular}{|c|c|c|c|c|c|c|c|}
\hline Cut & 0 jet & 1 jet & 2 jet & 3 jet & 4 jet & $\geq 5$ jets & all \\
\hline Total & 1497 & 27381 & 148347 & 354763 & 527883 & 786140 & 1846011 \\
\hline Good Run & 1349 & 24625 & 133800 & 319335 & 475961 & 708653 & 1663723 \\
\hline$\geq 1$ Tight Std. lepton & 803 & 13109 & 57758 & 88328 & 74486 & 22587 & 257071 \\
\hline \multicolumn{8}{|c|}{ CEM electrons } \\
\hline Tight Dilepton Veto & 238 & 4237 & 21301 & 40564 & 35141 & 10727 & 112208 \\
\hline Z Vertex Cut & 238 & 4237 & 21299 & 40561 & 35139 & 10726 & 112200 \\
\hline Z Boson Veto & 214 & 3894 & 19791 & 38556 & 34189 & 10397 & 107041 \\
\hline Missing $E_{\mathrm{T}}$ & 198 & 3586 & 18101 & 34701 & 30409 & 9195 & 96190 \\
\hline taggable & 0 & 2890 & 16702 & 33015 & 29292 & 8920 & 90819 \\
\hline $\mathrm{b} \operatorname{tag} \geq 1$ & 0 & 1350 & 9807 & 21277 & 20040 & 6169 & 58643 \\
\hline \multicolumn{8}{|c|}{ PHX electrons } \\
\hline Tight Dilepton Veto & 62 & 1024 & 5310 & 10025 & 8870 & 2615 & 27906 \\
\hline Z Vertex Cut & 62 & 1024 & 5310 & 10025 & 8870 & 2615 & 27906 \\
\hline Z Boson Veto & 56 & 940 & 4977 & 9561 & 8581 & 2544 & 26659 \\
\hline Missing $E_{\mathrm{T}}$ & 50 & 839 & 4479 & 8424 & 7431 & 2211 & 23434 \\
\hline taggable & 0 & 725 & 4295 & 8314 & 7360 & 2190 & 22884 \\
\hline $\mathrm{b} \operatorname{tag} \geq 1$ & 0 & 348 & 2581 & 5365 & 5166 & 1551 & 15011 \\
\hline \multicolumn{8}{|c|}{ CMUP muons } \\
\hline Tight Dilepton Veto & 118 & 2549 & 13343 & 25380 & 22009 & 6735 & 70134 \\
\hline Z Vertex Cut & 118 & 2549 & 13341 & 25377 & 22002 & 6734 & 70121 \\
\hline Z Boson Veto & 107 & 2375 & 12689 & 24639 & 21645 & 6621 & 68076 \\
\hline Missing $E_{\mathrm{T}}$ & 93 & 2181 & 11531 & 22000 & 19060 & 5882 & 60747 \\
\hline taggable & 0 & 1753 & 10697 & 20927 & 18347 & 5681 & 57405 \\
\hline $\mathrm{b} \operatorname{tag} \geq 1$ & 0 & 803 & 6281 & 13254 & 12625 & 3973 & 36936 \\
\hline \multicolumn{8}{|c|}{ CMX muons } \\
\hline Tight Dilepton Veto & 64 & 898 & 4527 & 8445 & 7409 & 2240 & 23583 \\
\hline Z Vertex Cut & 64 & 897 & 4525 & 8442 & 7406 & 2239 & 23573 \\
\hline Z Boson Veto & 56 & 844 & 4299 & 8208 & 7306 & 2201 & 22914 \\
\hline Missing $E_{\mathrm{T}}$ & 49 & 778 & 3895 & 7286 & 6454 & 1969 & 20431 \\
\hline taggable & 0 & 639 & 3664 & 6997 & 6281 & 1938 & 19519 \\
\hline $\mathrm{b} \operatorname{tag} \geq 1$ & 0 & 303 & 2188 & 4440 & 4360 & 1353 & 12644 \\
\hline \multicolumn{8}{|c|}{ All } \\
\hline Tight Dilepton Veto & 482 & 8708 & 44481 & 84414 & 73429 & 22317 & 233831 \\
\hline Z Vertex Cut & 482 & 8707 & 44475 & 84405 & 73417 & 22314 & 233800 \\
\hline Z Boson Veto & 433 & 8053 & 41756 & 80964 & 71721 & 21763 & 224690 \\
\hline Missing $E_{\mathrm{T}}$ & 390 & 7384 & 38006 & 72411 & 63354 & 19257 & 200802 \\
\hline taggable & 0 & 6007 & 35358 & 69253 & 61280 & 18729 & 190627 \\
\hline $\mathrm{b} \operatorname{tag} \geq 1$ & 0 & 2804 & 20857 & 44336 & 42191 & 13046 & 123234 \\
\hline
\end{tabular}

Table 3.3: Cut flow table of $t \bar{t}$ event selection for events of the $t \bar{t}$ signal MC sample ttopkl generated with Pythia. The different cuts are described in the text. 
the event cuts, designed to select $t \bar{t}$ events in the lepton+jets channel, are applied.

The requirement of at least one tight lepton causes the largest reduction of the initial number of events. Though in $66 \%$ of $t \bar{t}$ events at least one lepton is produced (see table 1.3), only in $15 \%$ of the events an electron or muon is found as tight lepton in the detector. The following cuts reject only few signal events and the number of rejected events due to a single cut depends on the order in which the cuts are applied. The last cut, the requirement of at least one $b$-tagged jet nearly halves the number of remaining signal events leading to an event acceptance of about $7 \%$ for $t \bar{t}$ events.

Table 3.4 presents the yield of events in the data sample selected in this way. The number of events obtained are shown separately for events, where the charged lepton is a CEM electron and where the charged lepton is a CMUP or CMX muon. In total we obtain $232 t \bar{t}$ candidates, 167 have one $b$ tag and 65 events are double tagged.

\begin{tabular}{|c|c|c|c|c|}
\hline $\mathcal{L}=955 \mathrm{pb}^{-1}$ & \multicolumn{3}{|c|}{ Charged lepton type } & $\overline{\text { All }}$ \\
\hline Sample & CEM & CMUP & CMX & leptons \\
\hline$N_{\mathrm{b}-\text { tags }}=1$ & 99 & 47 & 21 & 167 \\
\hline$N_{\text {b-tags }}>1$ & 42 & 15 & 8 & 65 \\
\hline$N_{\text {b-tags }} \geq 1$ & 141 & 62 & 29 & 232 \\
\hline
\end{tabular}

Table 3.4: Yield of $t \bar{t}$ candidates in the CDF data using an integrated luminosity of $955 \mathrm{pb}^{-1}$.

\subsection{Background Estimation}

The selected $t \bar{t}$ candidate sample still contains a certain level of background contamination. Among the 232 observed events, a background of $31.32 \pm 3.81$ events is predicted, following the background estimation of the $t \bar{t}$ cross section group [68]. The dominant sources are: $W$ production in association with heavy quarks and light quark production $(10.02 \pm 2.04)$, e.g. $\bar{q} q^{\prime} \rightarrow W g g$ with $g \rightarrow b \bar{b}(c \bar{c})$ and $g \rightarrow q^{\prime \prime} \bar{q}^{\prime \prime}$; so-called mistagged events $(12.25 \pm 1.83)$, in which a light quark jet is erroneously tagged as a $b$ jet; and events in which no real $W$ boson is produced $(6.80 \pm 1.8)$, e.g. direct $b \bar{b}$ production with additional gluon radiation. The latter are called non- $W$ or QCD events. Since in these processes no leptons are produced, one of the jets has to fake the charged lepton. In addition, electroweak processes, like single $Z$ boson, diboson (WZ, WW, ZZ), and single top quark production contribute to the background. However, the fraction of these backgrounds is rather small and can be determined based on their theoretical cross sections [69-71] and the acceptances and efficiencies derived from MC simulation and data. In table 3.5 a summary of the background estimation for a luminosity of $955 \mathrm{pb}^{-1}$ is given. 


\begin{tabular}{|c|c|}
\hline QCD $($ non $W)$ & $6.80 \pm 1.80$ \\
\hline Mistags & $12.25 \pm 1.83$ \\
\hline$W+c$ & $0.60 \pm 0.15$ \\
$W+c \bar{c}$ & $3.04 \pm 0.95$ \\
$W+b \bar{b}$ & $6.38 \pm 1.80$ \\
Diboson & $1.63 \pm 0.29$ \\
Single-Top & $0.62 \pm 0.10$ \\
\hline$\sum$ & $12.27 \pm 2.48$ \\
\hline \hline Total & $31.32 \pm 3.81$ \\
\hline
\end{tabular}

Table 3.5: Summary of background estimation for events with at least four jets for an integrated luminosity of $955 \mathrm{pb}^{-1}$. The errors for diboson and $W \bar{c}$ background are added linearly; all other errors are added in quadrature.

In order to model the background shape of differential distributions we use for the mistags and for the $W$ +heavy flavor background as well as for the diboson and single-top background two different Monte Carlo samples. For the mistag background we use the $W+$ four light quark jets sample and for the $W$ +heavy flavor background as well as for the diboson and single-top background we use the $W+$ two $b$ quark jets+two light quark jets sample.

In non- $W$ background events one of the produced jets is misinterpreted as a charged lepton and thus the event is accepted by our event selection. In order to simulate this background, we use a multi-jet data sample consisting of events with at least five jets. We then apply the complete lepton+jets event selection except the lepton ID request. If one of the jets has an electromagnetic fraction between 0.8 and 0.95, this jet is redefined as tight lepton. To pass the event selection the transverse momentum of this "lepton" is required to be greater than $20 \mathrm{GeV} / c$. The second difference to the default lepton+jets event selection is, that no $b$-tag is required, which would reduce the number of events in the selected sample. Instead we use all events considered as taggable and assign randomly the $b$-tag information to one of the jets in the event. 


\section{Chapter 4}

\section{Full Reconstruction of $t \bar{t}$ Events}

After having described the selection of events with a lepton+jets signature in the previous chapter, we introduce in this chapter the full reconstruction of $t \bar{t}$ pairs in the selected decay channel. During the reconstruction of $t \bar{t}$ pairs several ambiguities occur which lead in most cases to 24 different hypotheses for one $t \bar{t}$ event candidate. The selection of the correct hypothesis is the hardest issue concerning the full reconstruction of the $t \bar{t}$ event. In this chapter, we describe our method for the full reconstruction of $t \bar{t}$ events and on which basis we choose one event interpretation for every single event. Furthermore, the quality of our method is checked and a comparison between data and Monte Carlo prediction is presented.

\subsection{Full Reconstruction}

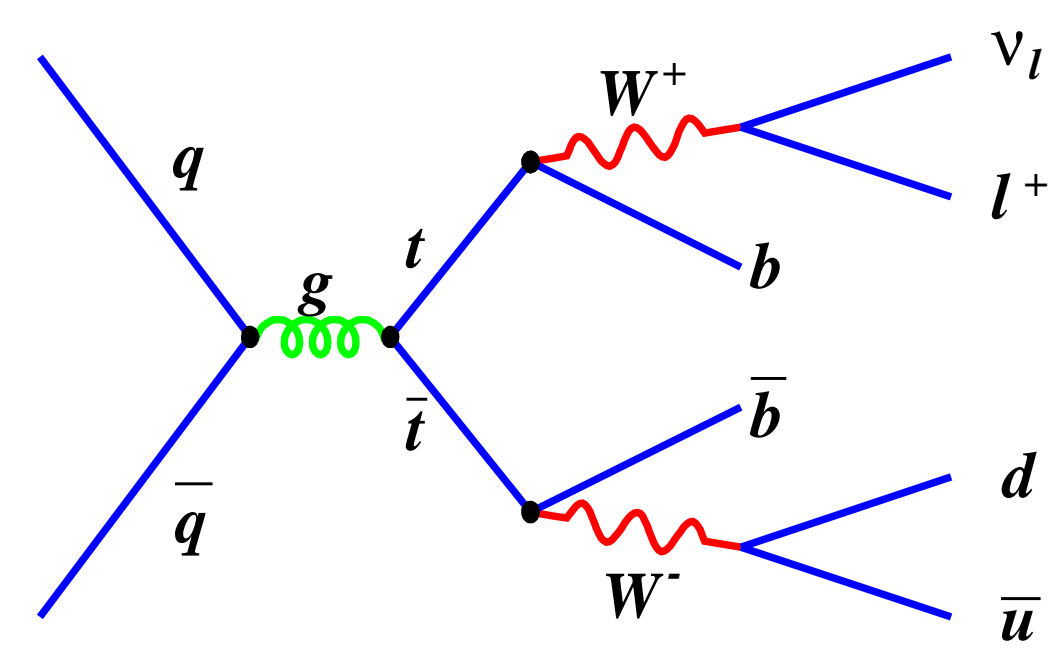

Figure 4.1: Feynman diagram of $t \bar{t}$ production via quark-antiquark annihilation and decay in the lepton+jets channel.

Figure 4.1 shows the Feynman diagram for a $t \bar{t}$ event in the lepton+jets channel. 
The most challenging issue is the assignment of the objects measured by the detector to the quarks and leptons from the top quark decay. Due to confinement quarks are not observed as free quarks but hadronize and therefore appear in the detector only in the form of jets. For the reconstruction of $t \bar{t}$ events the selected jets (level 4 correction) are corrected for further effects [66]. The calorimeter energy is corrected to the particle energy (level 5 correction), the underlying event energy is subtracted (level 6 correction) and finally out-of-cone corrections (level 7 corrections), leading from the particle energy to parton energy, are applied. The missing transverse energy is corrected only up to the sixth correction level to avoid double-counting. Since there are several possibilities for each single event to reconstruct the two top quarks from the decay particles, all hypotheses have to be reconstructed and afterwards one hypothesis has to be chosen.

\section{Leptonically decaying $W$ Boson: $W \rightarrow \ell \nu_{\ell}$}

The reconstruction of the $t \bar{t}$ pair starts with the charged lepton which can be reconstructed almost perfectly. As a second object the neutrino is reconstructed. Since the neutrino does not interact with the detector, it appears only in the missing transverse energy $\vec{E}_{\mathrm{T}}$. Thus, the transverse momentum of the neutrino, $\vec{P}_{\mathrm{T}, \nu}$, is given by $\vec{E}_{\mathrm{T}}$. Since $\vec{E}_{\mathrm{T}}$ lies by construction in the transverse plane, only the azimuthal angle and not the polar angle of $\vec{P}_{\nu}$ can be measured. The missing $z$ component of the momentum of the neutrino has to be calculated by using the $W$ mass constraint for the decay of the $W$ boson. Exploiting the fact, that the $W$ boson with a mass of $m_{W}=80.4 \mathrm{GeV} / c^{2}$ decays into a charged lepton and a neutrino, a quadratic equation in the $z$ component $P_{z, \nu}$ of the neutrino momentum is obtained:

$$
P_{z, \nu}^{2}-2 \cdot \frac{\mu \cdot P_{z, \ell}}{E_{\ell}^{2}-P_{z, \ell}^{2}} \cdot P_{z, \nu}+\frac{E_{\ell}^{2} \cdot P_{\mathrm{T}, \nu}^{2}-\mu^{2}}{E_{\ell}^{2}-P_{z, \ell}^{2}}=0
$$

Here $P_{z, \ell}$ and $E_{\ell}$ denote the $z$ component of the momentum and the energy of the charged lepton (electron or muon) respectively. The quantity $\mu$ is defined via $m_{W}^{2} / 2+\cos (\Delta \Phi) \cdot P_{\mathrm{T}, \ell} \cdot P_{T, \nu}$, in which $\Delta \Phi$ is the azimuthal angle difference between the momentum of the charged lepton and $\vec{E}_{\mathrm{T}}$. In general, a quadratic equation leads to two solutions. If the solution is complex we take the real part of the solution, otherwise both solutions have to be considered. Complex solutions occur, when the transverse mass of the $W$ boson, which is defined via $m_{\mathrm{T}, W}^{2}=$ $\left(P_{\mathrm{T}, \ell}+P_{\mathrm{T}, \nu}\right)^{2}-\left(P_{x, \ell}+P_{x, \nu}\right)^{2}-\left(P_{y, \ell}+P_{y, \nu}\right)^{2}$, is larger than the mass demanded by the $W$ mass constraint. MC studies show, that in $71 \%$ of all events with two real solutions the solution with the smaller value of $\left|P_{z, \nu}\right|$ leads to the correct $W$ boson four-vector. This is reasonable, since the decay products of heavy particles like the $W$ boson are dominantly produced with large transverse momenta and thus are central in the detector.

The four-momentum of the leptonically decaying $W$ boson is then obtained by adding the four-momenta of the charged lepton and of one of the two neutrino hypotheses. After the reconstruction the four-vector of the leptonically decaying $W$ 
boson has to be corrected. In case of a complex neutrino solution which occurs in approximately $30 \%$ of all events only the real part of the solution is taken. Since the real part alone does not fulfill the quadratic equation 4.1 completely, the mass of the reconstructed $W$ boson is no longer equal to the mass constraint of $80.4 \mathrm{GeV} / c^{2}$. On that account we have to recalculate the energy of the reconstructed leptonically decaying $W$ boson to force $m_{W}=80.4 \mathrm{GeV} / c^{2}$.

\section{Semileptonically decaying Top Quark: $t \rightarrow b \ell \nu_{\ell}$}

The next step is the reconstruction of the semileptonically decaying top quark. All hypotheses obtained by adding the four-momentum of each selected jet and the four-momentum of the leptonically decaying $W$ boson are considered. In total, the number of hypotheses is equal to the number of reconstructed jets $N_{\text {jets }}$ times the number of solutions for the $z$ component of the neutrino momentum.

\section{Hadronically decaying $W$ Boson: $W \rightarrow j j$}

The four-momentum of the hadronically decaying $W$ boson is then obtained by combining the four-momenta of two of the selected jets which are not assigned to the semileptonically decaying top quark in the hypothesis considered. This leads to $\left(N_{\text {jets }}-1\right) \cdot\left(N_{\text {jets }}-2\right)$ combinations for the reconstruction of the hadronically decaying $W$ boson. Due to the fact that the permutation of the two chosen jets leads to the same $W$ boson four-momentum, we only consider one of the two possibilities to arrange the two jets, which reduces the combinatorics by a factor of 2 .

\section{Hadronically decaying Top Quark: $t \rightarrow b j j$}

The last step is the assignment of a jet to the $b$ jet from the hadronic top decay. Those jets are considered that are not assigned so far to a light jet from the hadronically decaying $W$ boson or the $b$ jet from the semileptonically decaying top quark. The four-momentum of one of the $\left(N_{\text {jets }}-3\right)$ remaining jets is then added to the fourmomentum of the hadronically decaying $W$ boson resulting in the four-momentum of the hadronically decaying top quark.

In total, this leads to $N_{\text {jets }} \cdot\left(N_{\text {jets }}-1\right) \cdot\left(N_{\text {jets }}-2\right) \cdot\left(N_{\text {jets }}-3\right) / 2$ hypotheses for the assignment of the jets and due to the two solutions of the $z$ component of the neutrino momentum to $N_{\text {jets }} \cdot\left(N_{\text {jets }}-1\right) \cdot\left(N_{\text {jets }}-2\right) \cdot\left(N_{\text {jets }}-3\right)$ hypotheses for the complete kinematic reconstruction of a $t \bar{t}$ event candidate. For example an event containing four jets and two neutrino solutions leads to 24 different event interpretations. Although this leads to large amounts of hypotheses for events with more than four jets it is reasonable to consider all jets of one event, since MC studies show, that only in $37 \%$ of the cases the correct event interpretation consists of the four leading jets, i.e. the four jets with the highest energies. 


\subsection{Selection of one Event Interpretation}

In order to analyze the selected data sample, one hypothesis has to be chosen for every single event. For MC events it is possible to determine the hypothesis which is closest to the true event. This best possible hypothesis is defined as the hypothesis for which the deviation of the reconstructed top quarks and $W$ bosons from the generated objects in the $\eta-\phi$ plane is minimal (for a detailed description of this definition see section 4.3). It is obvious that when dealing with real events this criterion cannot be adopted, since no information about the true four-vectors of the top quarks and $W$ bosons is accessible. Therefore, we determine for each hypothesis a quantity $\Psi$ which gives a quantitative estimate how well the hypothesis matches the $t \bar{t}$ pair assumption and choose the hypothesis with the smallest value of $\Psi$. Constraints on the mass of the hadronically decaying $W$ boson and on the difference between both reconstructed top quark masses, on the $b$-likeness of the jets chosen to be the $b$ jets from the $t \bar{t}$ pair as well as a constraint on the sum of the reconstructed transverse energy of the two top quarks which should in leading order calculation be equal to the transverse energy of the event, enter the calculation of $\Psi$. The quantity $\Psi$ is defined as:

$$
\Psi=P_{\nu} \cdot \chi^{2} \cdot P_{\mathrm{b}-\text { light }}
$$

\section{Neutrino Momentum $z$ Component Solution: $P_{\nu}$}

As mentioned, in general two solutions exist for $P_{z, \nu}$. Due to the fact, that in $71 \%$ of all cases the solution with the smaller value of $\left|P_{z, \nu}\right|$ leads to the correct $W$ boson four-vector, we introduce a weighting factor $P_{\nu}$ that is 0.29 for hypotheses with the smaller absolute value of $P_{z, \nu}$ and 0.71 for hypotheses with the larger value for $P_{z, \nu}$. Thus, $P_{\nu}$ can be interpreted as the probability for the chosen neutrino solution to be the wrong one.

\section{Mass and Energy Constraints: $\chi^{2}$}

The constraints on the mass of the hadronically decaying $W$ boson, on the difference between the two top quark masses and on the transverse energy of the $t \bar{t}$ pair are considered in a $\chi^{2}$ function which is defined via:

$$
\chi^{2}=\frac{\left(m_{W \rightarrow j j}-\bar{m}_{W \rightarrow j j}\right)^{2}}{\sigma_{m_{W \rightarrow j j}}^{2}}+\frac{\left(m_{t \rightarrow b \ell \nu}-m_{t \rightarrow b j j}\right)^{2}}{\sigma_{\Delta m_{t}}^{2}}+\frac{\left(P_{\text {energy }}-\bar{P}_{\text {energy }}\right)^{2}}{\sigma_{P_{\text {energy }}}^{2}}
$$

Three terms contribute to the computation of $\chi^{2}$. In the first term $m_{W \rightarrow j j}$ is the reconstructed mass of the hadronically decaying $W$ boson, which should be equal to the mean value $\bar{m}_{W \rightarrow j j}$ of the $m_{W \rightarrow j j}$ distribution within the resolution $\sigma_{m_{W \rightarrow j j}}$. In the second term $\Delta m_{t}$ is the difference between the reconstructed mass of the semileptonically decaying top quark $m_{t \rightarrow b \ell \nu}$ and the mass of the hadronically 
decaying top quark $m_{t \rightarrow b j j}$. Since these are two identical particles, the difference should be equal to zero. The mean value $\bar{m}_{W \rightarrow j j}$ and the widths $\sigma_{m_{W \rightarrow j j}}$ and $\sigma_{\Delta m_{t}}$ are obtained from the Gaussian with the smaller width of a double Gaussian fit to the corresponding mass distributions obtained from MC simulation, where the best possible hypothesis for every event is taken. The distributions are presented in figure 4.2(a) and (b). The values are:

$$
\begin{aligned}
\bar{m}_{W \rightarrow j j} & =79.5 \mathrm{GeV} / c^{2}, \\
\sigma_{m_{W \rightarrow j j}} & =10.6 \mathrm{GeV} / c^{2}, \\
\sigma_{\Delta m_{t}} & =25.8 \mathrm{GeV} / c^{2} .
\end{aligned}
$$

The asymmetric shape of the $\Delta m_{t}$ distribution is due to the different detector performance in the energy resolution between jets and leptons. Since the four-vector of the hadronically decaying top quark consists of three jets and the four-vector of the semileptonically decaying top quark consists only of one jet, the reconstructed masses show a small discrepancy.

In the third term $P_{\text {energy }}$ is the sum of the transverse energies of the two top quarks divided by the total transverse energy of the event including missing transverse energy:

$$
P_{\text {energy }}=\frac{\sqrt{p_{\mathrm{T}, t \rightarrow b \ell \nu}^{2}+m_{t \rightarrow b \ell \nu}^{2}}+\sqrt{p_{\mathrm{T}, t \rightarrow b j j}^{2}+m_{t \rightarrow b j j}^{2}}}{\sum_{\text {jets }} p_{\mathrm{T}, \mathrm{jet}}+\mathbb{E}_{\mathrm{T}}+E_{\mathrm{T}, \ell}} .
$$

Here $p_{\mathrm{T}, t \rightarrow b \ell \nu}$ and $p_{\mathrm{T}, t \rightarrow b j j}$ represent the reconstructed transverse momenta of the semileptonically and hadronically decaying top quark and $m_{t \rightarrow b \ell \nu}$ and $m_{t \rightarrow b j j}$ are the reconstructed top quark masses. $p_{\mathrm{T} \text {,jet }}$ is the transverse momentum of a jet, $\mathbb{E}_{\mathrm{T}}$ is the measured missing transverse energy and $E_{\mathrm{T}, \ell}$ is the transverse energy of the charged lepton. For the best possible event interpretation we fit a double Gaussian to the $P_{\text {energy }}$ distribution displayed in figure 4.2 (c) and obtain a mean of 1.102 and a width of 0.07 for the Gaussian with the smaller width $\sigma$. The mean of the $P_{\text {energy }}$ distribution is indicated in equation 4.3 with $\bar{P}_{\text {energy }}$.

\section{$b$ Probability of $b$ Jets $: P_{\mathrm{b}-\text { light }}$}

The quantity $P_{\mathrm{b}-\text {-light }}$ is a measure for the light quark likeness of the jets assigned as $b$ jets and is defined as:

$$
P_{\mathrm{b}-\text { light }}=\left(\mathcal{P}_{t \rightarrow b l \nu}+\left(1-R_{t \rightarrow b l \nu}^{\prime}\right)\right) \cdot\left(\mathcal{P}_{t \rightarrow b j j}+\left(1-R_{t \rightarrow b j j}^{\prime}\right)\right) .
$$

Here $\mathcal{P}_{t \rightarrow b \ell \nu}$ and $\mathcal{P}_{t \rightarrow b j j}$ are the probabilities of the jets chosen to be the $b$ jets from the semileptonically and hadronically decaying top quark, respectively, to belong to the primary vertex. This probability is calculated with the JetProb package [72] and is based on the positive impact parameter of the tracks assigned to the jet in the $r$ - $\phi$-plane. 
(a)

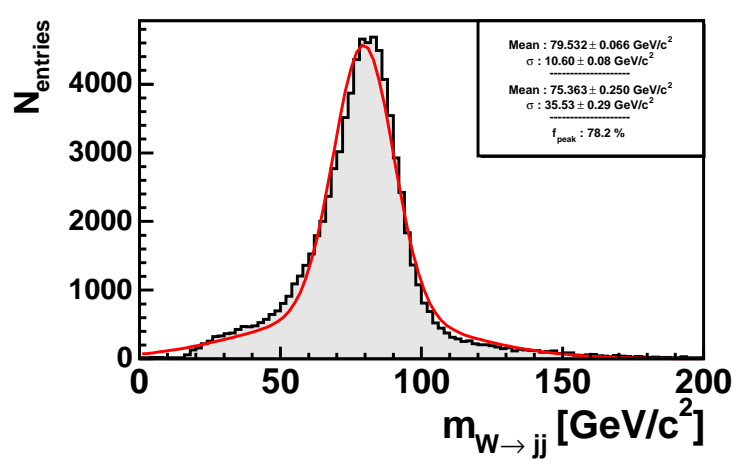

(c)

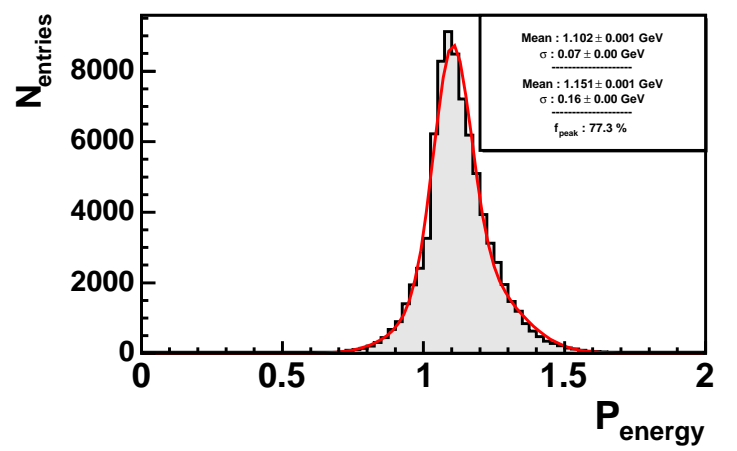

(b)

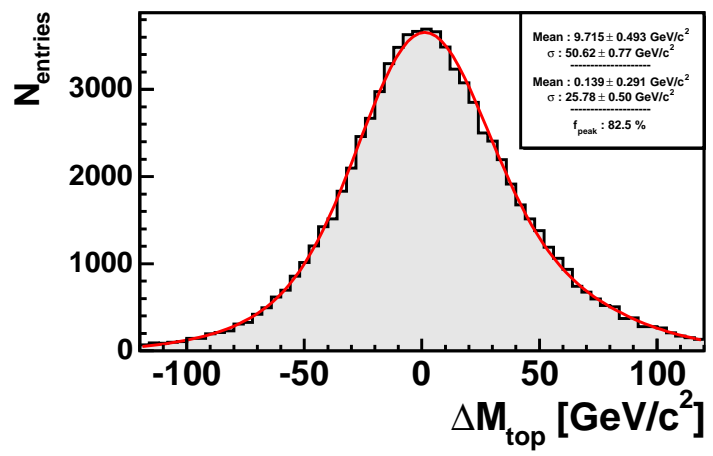

Figure 4.2: Determination of the input parameters of the $\chi^{2}$ function: (a) $\bar{m}_{W \rightarrow j j}$ and $\sigma_{m_{W \rightarrow j j}}$, (b) $\sigma_{\Delta m_{t}}$, (c) $\bar{P}_{\text {energy }}$ and $\sigma_{P_{\text {energy }}}$. A double Gaussian is fitted (a) to the distribution of the $W$ boson mass $m_{W \rightarrow j j}$, (b) to the distribution of the top quark mass difference $\Delta m_{t}$, and (c) to the distribution of $P_{\text {energy }}$ obtained from the best possible hypotheses. $\bar{m}_{W \rightarrow j j}$, and $\bar{P}_{\text {energy }}$ are the mean values of the Gaussian with the smaller width. $\sigma_{m_{W \rightarrow j j}}, \sigma_{\Delta m_{t}}$ and $\sigma_{P_{\text {energy }}}$ are the widths $\sigma$ of the narrower Gaussians. In addition, the fraction of events $f_{\text {peak }}$ contained in the peak, meaning events within $2 \sigma$ of the narrower Gaussian, is stated. 
In order to decide how much a hypothesis in which the jet assigned as $b$ jet has a SecVtx [67] tag should be preferred, we estimate the quality $R^{\prime}$ of the $b$-tag using a neural network (NN) $b$-tagger [73] designed for the single-top search. Since $P_{\mathrm{b} \text {-light }}$ is defined as the probability for the assigned $b$ jets to be light quark jets, we have to use $\left(1-R^{\prime}\right)$ instead of $R^{\prime}$ in equation $4.5 . R^{\prime}$ is defined as:

$$
R^{\prime}=\frac{1}{1+\left(\frac{1}{R}-1\right) \cdot \frac{B^{\prime} \cdot S}{S^{\prime} \cdot B}}
$$

The probability $R$ for a tagged jet to be a $b$ jet can be computed by multiplying the output of the NN $b$-tagger, a value between -1 and +1 , by 0.5 and adding 0.5 if the signal to background ratio in the data is the same as in the training samples, the neural net was trained with. The signal fraction in the training samples is indicated by $S$, the background fraction by $B$. In this case "signal" means all processes in which $b$ jets are produced, $t \bar{t}$ as well as for example $W+b \bar{b}$ or single-top production. Since the NN b-tagger is designed for the search of single-top quark production, the neural net was trained with the expected signal to background ratio for lepton + jets events with not more than three jets. Due to the different signal to background ratio in the data sample used for this analysis, $R$ has to be modified by considering the expected background fraction $B^{\prime}$ and signal fraction $S^{\prime}$ for lepton+jets events with at least four jets, which is done in equation 4.6. Starting from the fact, that the ratio of the measured probabilities to be background $(1-R)$ or signal $(R)$ times the ratio of the a priori probabilities for signal $(S)$ and background $(B)$ has to be the same for different a priori probabilities, we obtain a relation between $R$ and $R^{\prime}$.

$$
\frac{1-R^{\prime}}{R^{\prime}} \cdot \frac{S^{\prime}}{B^{\prime}}=\frac{1-R}{R} \cdot \frac{S}{B}
$$

This relation leads directly to equation 4.6 (see also figure 4.3).

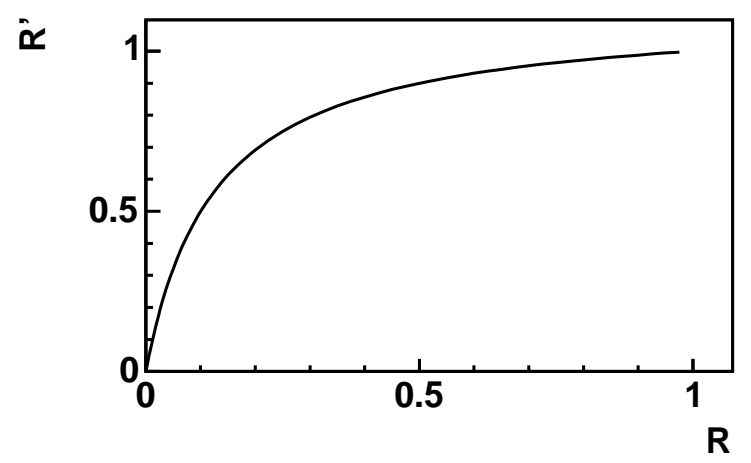

Figure 4.3: Modification of the probability $R$ due to different signal to background ratios in the training samples and in data. Signal fraction in the training samples $(S): 0.586$, expected signal fraction in the data $\left(S^{\prime}\right): 0.927$, background fraction in the training samples $(B): 0.414$, expected background fraction in the data $\left(B^{\prime}\right): 0.073$. 
As mentioned for our analysis we choose the event interpretation with the smallest value of $\Psi$. For the selected hypothesis as well as for all other hypotheses the four-vectors of the top quark and antitop quark are corrected. The momentum components remain unchanged, while the energy is recalculated to force the top quark mass to be $m_{t}=175 \mathrm{GeV} / c^{2}$ instead of the originally reconstructed mass. For the measurement of the top mass distribution the unmodified reconstructed four-momentum vector is used.

\subsection{Best possible Event Interpretation in MC}

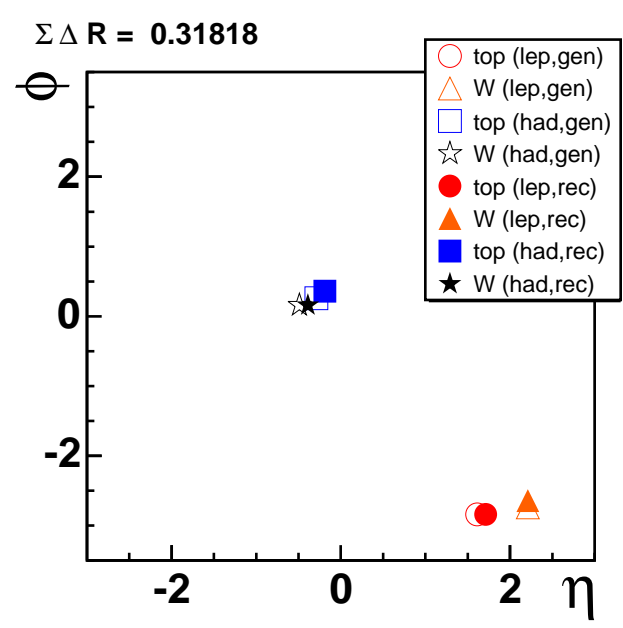

(a)

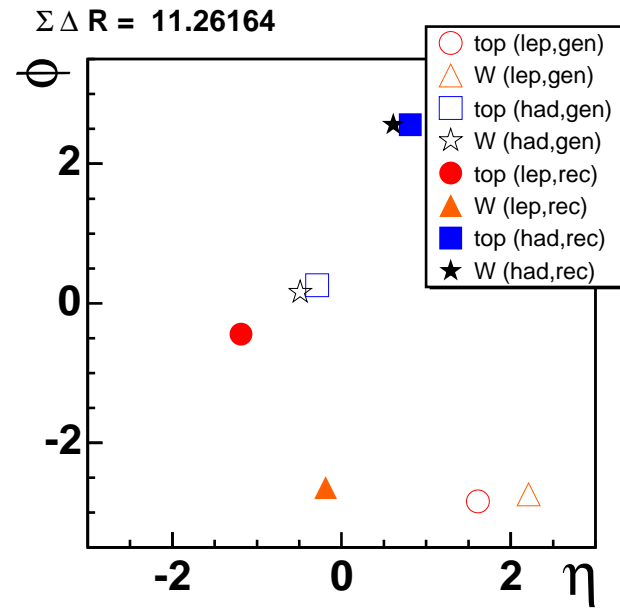

(b)

Figure 4.4: Position of the generated (referred to as "gen") and reconstructed (referred to as "rec") four-vectors of the two $W$ bosons (the leptonically decaying $W$ boson is indicated with "lep", the hadronically decaying with "had") and top quarks (same notation as for the $W$ bosons) in the $\eta-\phi$ plane (a) for the best possible hypothesis and (b) for one of the other 23 event interpretations of the same event together with the corresponding values for $\sum \Delta R$ (for explanation see eq. 4.8).

In simulated $\mathrm{MC}$ events it is possible to determine that hypothesis with the smallest deviation to the generated $t \bar{t}$ pair. This hypothesis we call best possible hypothesis and it is defined as the hypothesis for which the sum of the distances in the $\eta$ - $\phi$ plane of the leptonically decaying $W$ boson $\Delta R_{W \rightarrow \ell \nu}$, the semileptonically decaying top quark $\Delta R_{t \rightarrow b \ell \nu}$, the hadronically decaying $W$ boson $\Delta R_{W \rightarrow j j}$, and the hadronically decaying top quark $\Delta R_{t \rightarrow b j j}$ to the corresponding generated objects has a minimum value:

$$
\sum \Delta R=\Delta R_{W \rightarrow \ell \nu}+\Delta R_{t \rightarrow b \ell \nu}+\Delta R_{W \rightarrow j j}+\Delta R_{t \rightarrow b j j}
$$

The distance $\Delta R$ between two objects is given by $\Delta R=\sqrt{\Delta \phi^{2}+\Delta \eta^{2}}$, where $\Delta \phi$ is the azimuthal angle difference between those objects and $\Delta \eta$ is the difference be- 

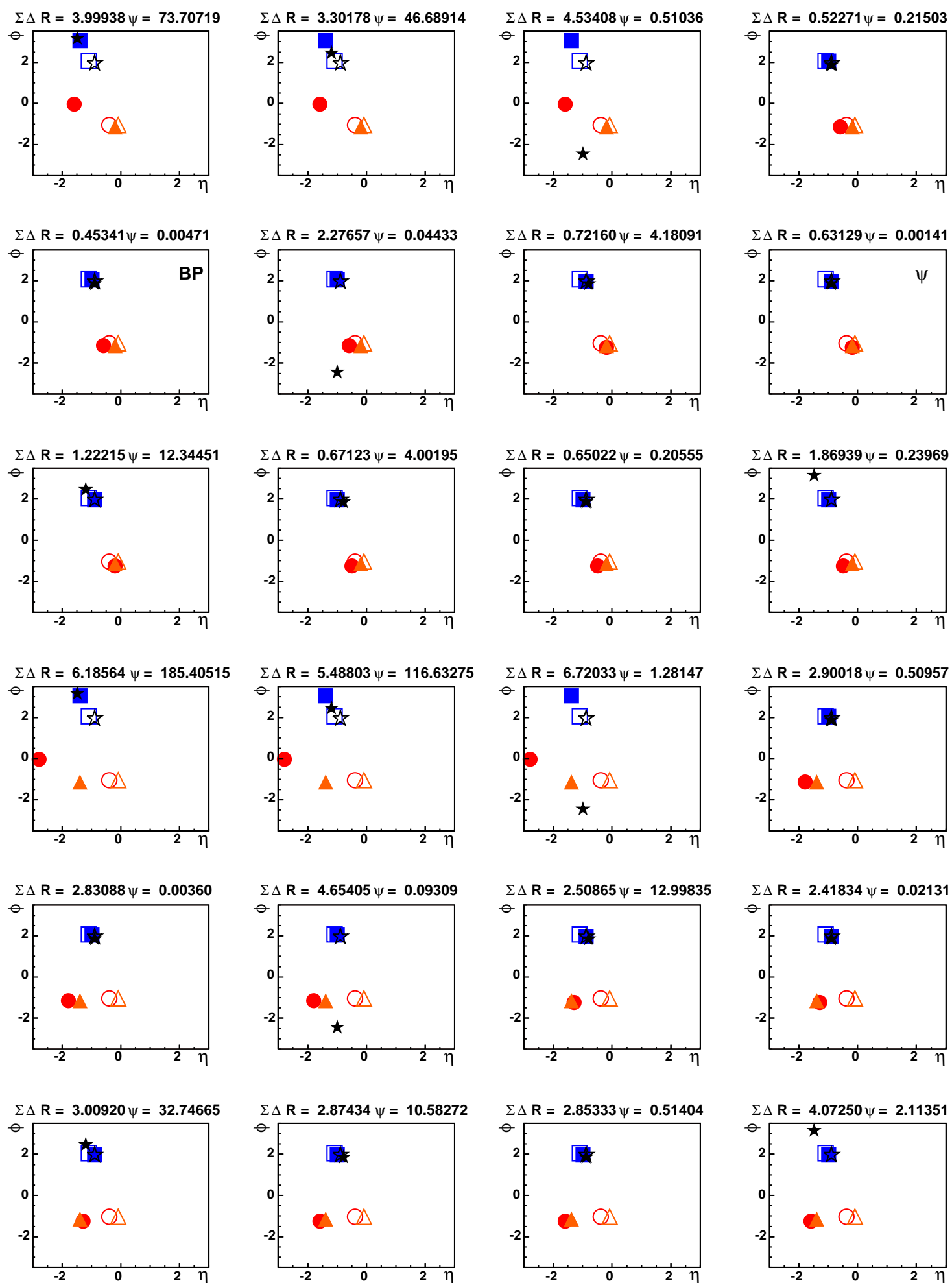

Figure 4.5: $\eta-\phi$ plots for all 24 hypothesis of a certain event. The best possible one is indicated by the index "BP", the chosen one by " $\Psi$ " - in this event the selected one is not the best possible, but very similar to the best possible. 
tween both pseudorapidities. The motivation for this definition is the fact that for every event there exists one hypothesis which is as close as possible to the true event topology, i.e. the generated $t \bar{t}$ pair in case of MC studies. Also if the jet energies or directions are not well measured by the detector or only three jets originating from the four quarks are reconstructed but an additional jet originating from gluon radiation is found, there is one way to arrange the given four-vectors that leads to the event interpretation closest to the truth. The definition of $\Delta R$ is illustrated in figure 4.4 in which the position in the $\eta$ - $\phi$ plane of the four-vectors of the two $W$ bosons and top quarks are shown for the reconstructed objects as well as for the generated ones. In figure 4.5 this plot is shown for all 24 hypotheses for a certain event. In addition, the values for $\sum \Delta R$ and for $\Psi$ are presented. The best possible hypothesis is indicated by "BP", the hypothesis that is chosen on the basis of our selection is indicated by " $\Psi$ ".

\subsection{Performance of our Selection}

In the previous section the best possible hypothesis for generated events was introduced. The quality of our selection based on the quantity $\Psi$ is now checked by comparing the selected event interpretation with the best possible one and the MC true values.

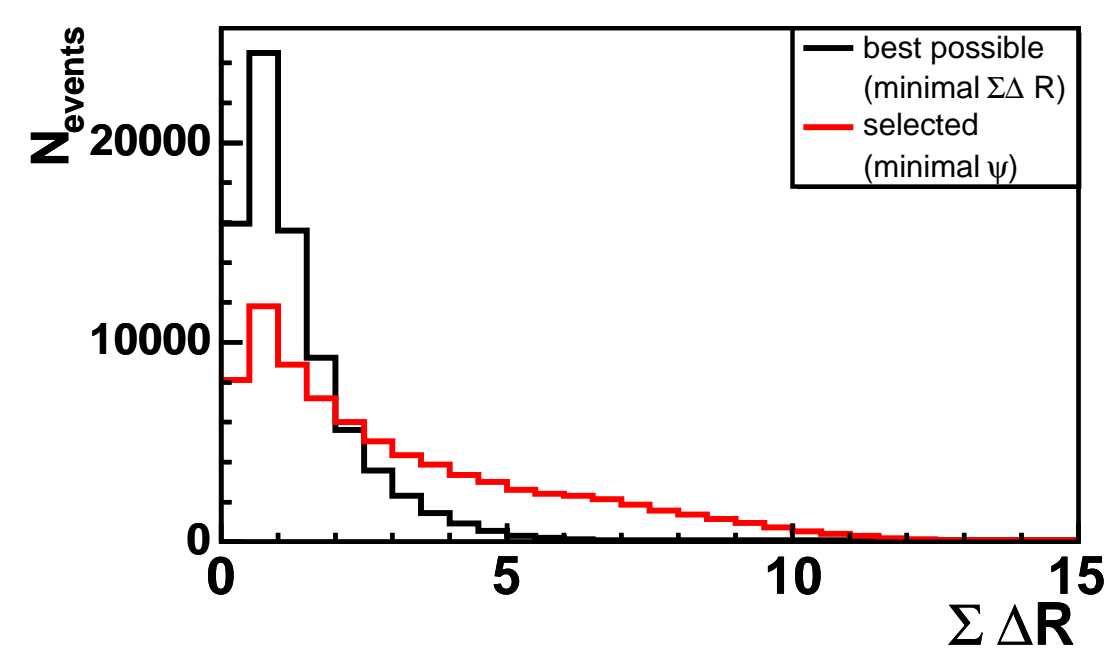

Figure 4.6: Performance of the hypothesis selection. Shown are the $\sum \Delta R$ distributions for the selected hypotheses based on the minimal $\Psi$ and for the best possible hypotheses. The distributions are obtained from a PYTHIA MC sample. 


\begin{tabular}{c|c} 
& $\begin{array}{c}\text { Selected } \boldsymbol{t} \bar{t} \\
\text { hypothesis [\%] }\end{array}$ \\
\hline best possible & $34.06 \pm 0.17$ \\
$\sum \Delta R<2.0$ & $44.25 \pm 0.18$ \\
$\sum \Delta R<4.0$ & $68.17 \pm 0.16$ \\
$\sum \Delta R<6.0$ & $82.55 \pm 0.13$ \\
\hline
\end{tabular}

Table 4.1: Quality of the hypothesis selection. The fraction of selected hypotheses which correspond to the best possible hypotheses is stated as well as the fractions of selected hypotheses within a certain distance $\sum \Delta R$ with respect to the Monte Carlo true values.

Figure 4.6 provides the $\sum \Delta R$ distribution for the chosen hypotheses based on the minimum value of $\Psi$ as well as for the best possible hypotheses (in each event the hypothesis with the smallest value of $\sum \Delta R$ ) obtained from a PYTHIA MC $t \bar{t}$ sample. The minimal value of $\sum \Delta R$ can vary from event to event and though for the most events this minimum takes values around one, in some events it reaches up to five and more. In addition, table 4.1 shows how often the selected hypothesis for the $t \bar{t}$ pair corresponds to the best possible event interpretation. It is also stated how often our selected hypothesis lies within a certain distance $\sum \Delta R$ with respect to the Monte Carlo true values. In about $34 \%$ of all events the selected hypothesis corresponds exactly to the best possible hypothesis, while in $83 \%$ of all cases $\sum \Delta R$ is below 6.0 .

Figures 4.7 - 4.10 show some distributions of kinematic quantities for the selected hypotheses compared to the distributions for the best possible hypotheses and for all other hypotheses which are weighted by the number of event hypotheses subtracted by one (best possible hypothesis). "All other" hypotheses are all hypotheses of an event except the best possible hypothesis. Thus they include hypotheses with completely wrong combinations of jets and leptons as well as hypotheses with partial correct assignments in which for example only one top quark is correctly reconstructed.

In figures 4.7 and 4.8 the transverse momentum $p_{\mathrm{T}}$ and the pseudorapidity $\eta$ of the leptonically decaying $W$ boson and the hadronically decaying $W$ boson as well as the pseudorapidity of the neutrino are presented. In case of the hadronically decaying $W$ boson the reconstructed mass distribution is shown in addition. The transverse momentum distribution of the leptonically decaying $W$ boson for the selected hypotheses is equal to that for the best possible ones and to that for all other hypotheses, since the transverse components are reconstructed very precisely. In case of the pseudorapidity of the neutrino a bias of the distribution obtained from the selected hypotheses towards smaller absolute values of $\eta$ can be seen. This effect is due to the preference of the neutrino solution with the smaller magnitude of $P_{z, \nu}$ and therefore also occurs in the $\eta$-distribution of the leptonically decaying $W$ boson. In general, the distributions obtained from the selected hypotheses follow well the distributions of the best possible hypotheses. In case of the reconstructed mass of 
(a)

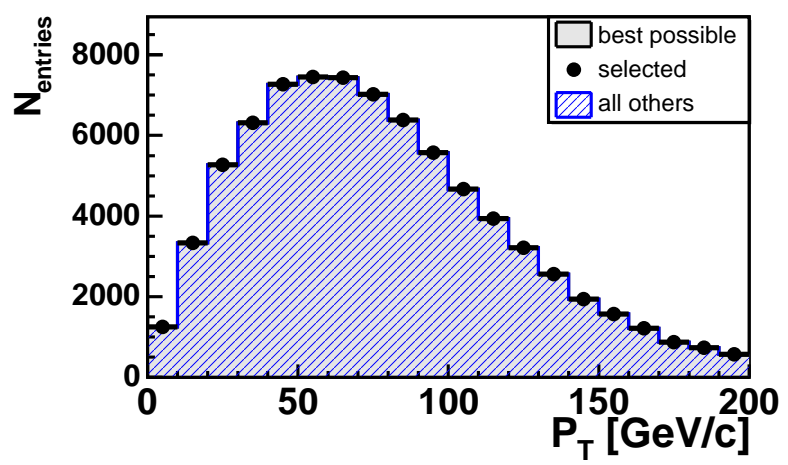

(c) (b)

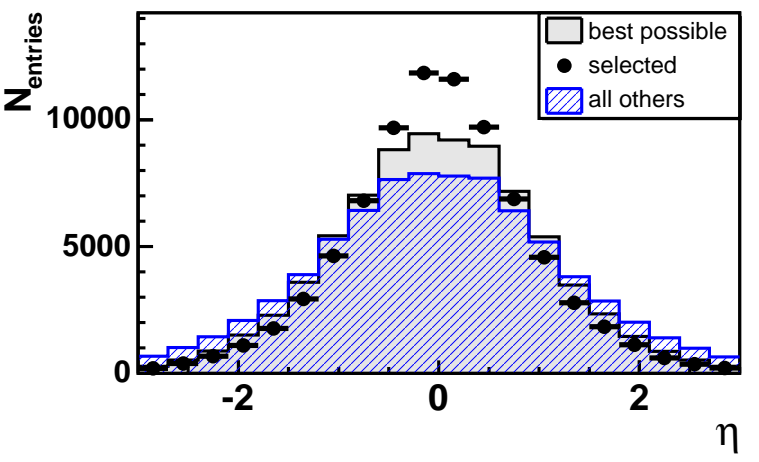

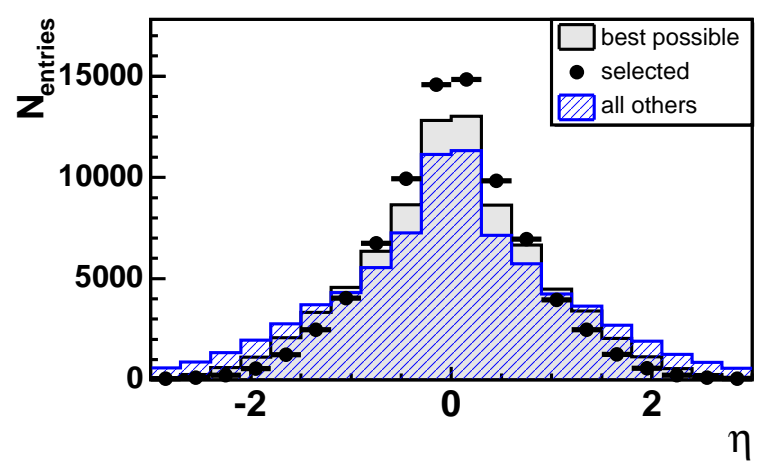

Figure 4.7: Distributions of kinematic quantities of the leptonically decaying $\boldsymbol{W}$ boson: $\boldsymbol{W} \rightarrow \boldsymbol{l} \boldsymbol{\nu}$ and the neutrino $\boldsymbol{\nu}$ obtained from a PYTHIA MC sample: (a) transverse momentum and (b) pseudorapidity of the $W$ boson, (c) pseudorapidity of the neutrino. The selected hypothesis (black points) is compared with the best possible hypothesis (grey, see text for explanation) and all other hypotheses (blue hatched) which are weighted by the number of event hypotheses subtracted by one (best possible hypothesis). 
(a)

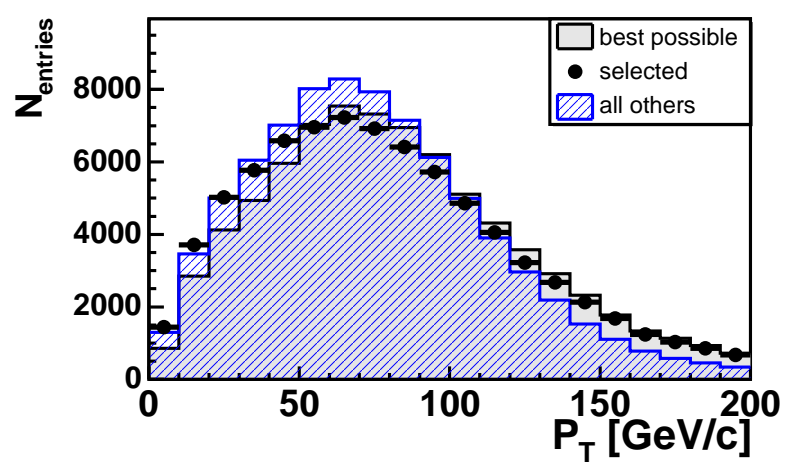

(c)

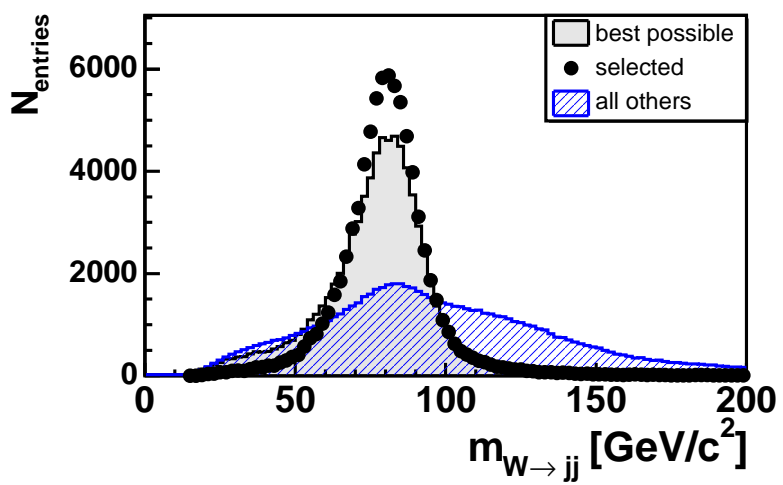

(b)

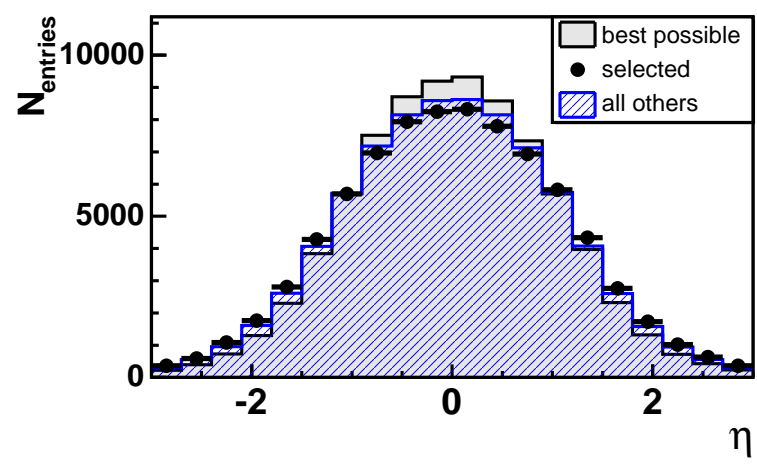

Figure 4.8: Distributions of kinematic quantities of the hadronically decaying $\boldsymbol{W}$ boson: $\boldsymbol{W} \rightarrow \boldsymbol{j} \boldsymbol{j}$ obtained from a PYтнIA MC sample: (a) transverse momentum, (b) pseudorapidity, and (c) reconstructed mass of the $W$ boson. The selected hypothesis (black points) is compared with the best possible hypothesis (grey, see text for explanation) and all other hypotheses (blue hatched) which are weighted by the number of event hypotheses subtracted by one (best possible hypothesis). 
(a)

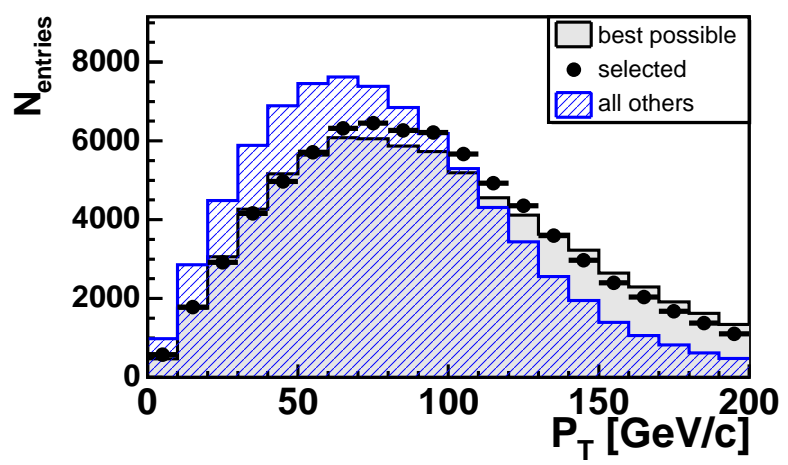

(c)

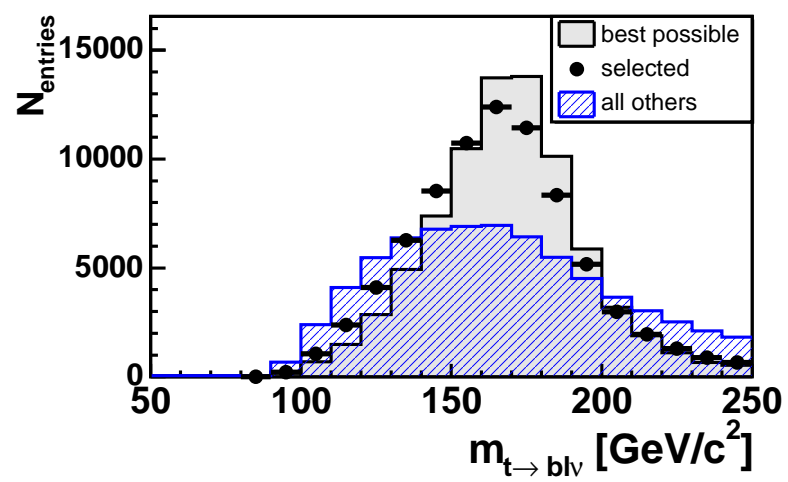

(b)

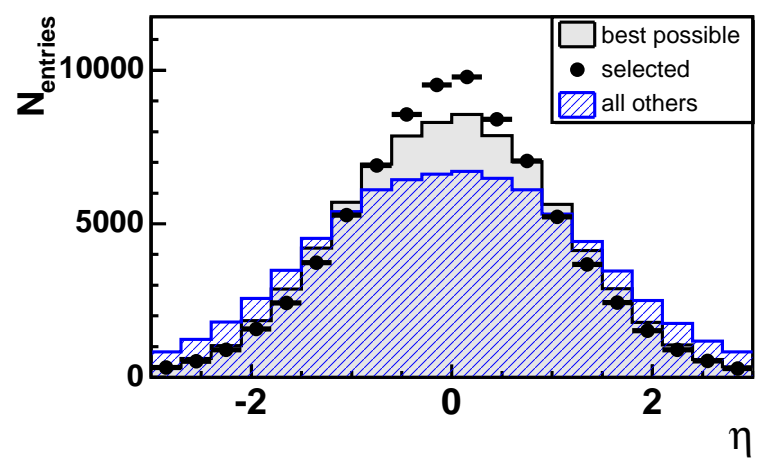

Figure 4.9: Distributions of kinematic quantities of the semileptonically decaying top quark: $\boldsymbol{t} \rightarrow \boldsymbol{b} \boldsymbol{l} \boldsymbol{\nu}$ obtained from a PYTHIA MC sample: (a) transverse momentum, (b) pseudorapidity, and (c) reconstructed mass of the top quark. The selected hypothesis (black points) is compared with the best possible hypothesis (grey, see text for explanation) and all other hypotheses (blue hatched) which are weighted by the number of event hypotheses subtracted by one (best possible hypothesis). 
(a)

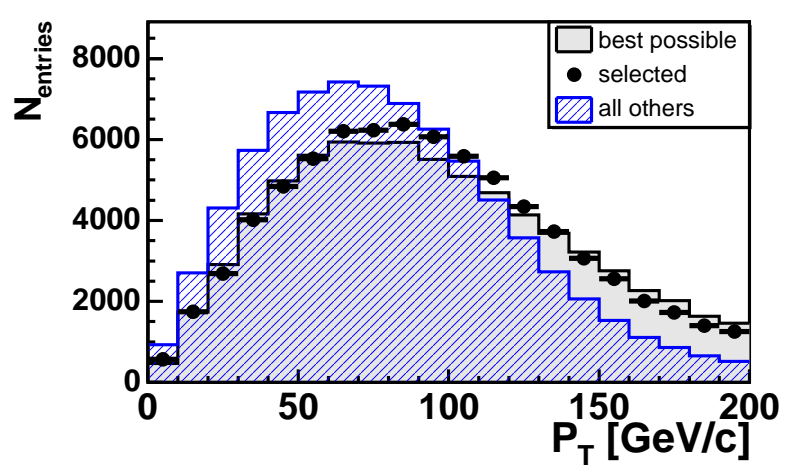

(c)

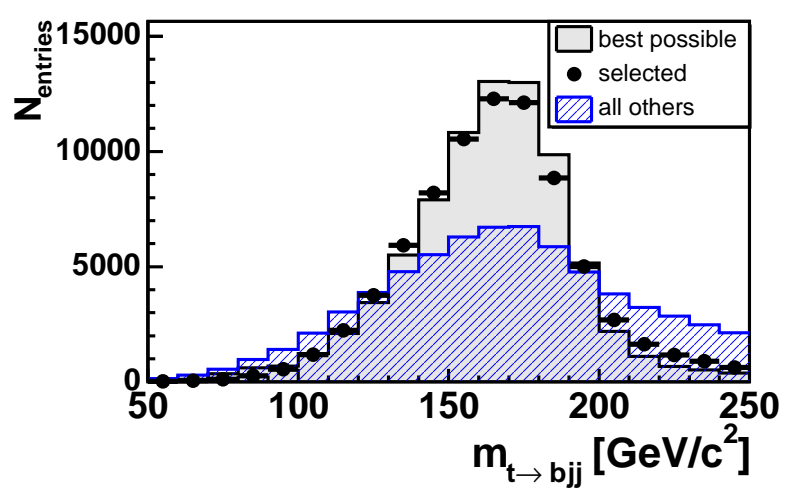

(b)

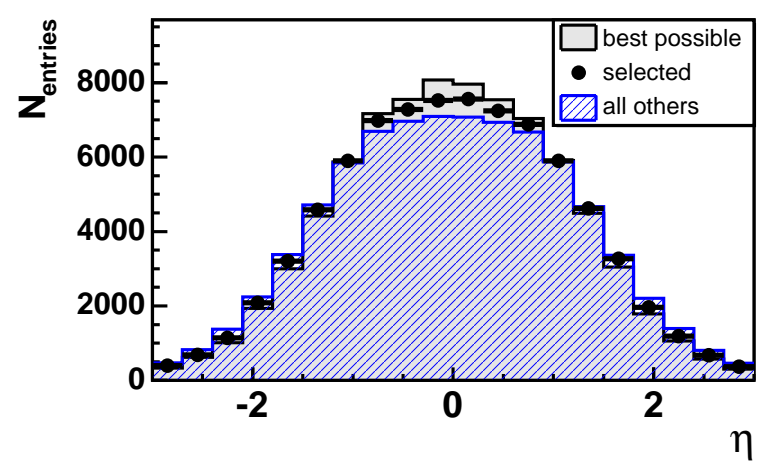

Figure 4.10: Distributions of kinematic quantities of the hadronically decaying top quark: $\boldsymbol{t} \boldsymbol{\rightarrow} \boldsymbol{b} \boldsymbol{j} \boldsymbol{j}$ obtained from a PүтніA MC sample: (a) transverse momentum, (b) pseudorapidity, and (c) reconstructed mass of the top quark. The selected hypothesis (black points) is compared with the best possible hypothesis (grey, see text for explanation) and all other hypotheses (blue hatched) which are weighted by the number of event hypotheses subtracted by one (best possible hypothesis). 
the hadronically decaying $W$ boson the shape of the distribution for all other hypotheses is substantially broader than that of the best and of the selected hypotheses.

For the top quarks the transverse momentum $p_{\mathrm{T}}$ and the pseudorapidity $\eta$ are shown in figures 4.9 and 4.10. In addition, the mass distributions of both top quarks are presented. Again distributions obtained from the selected hypotheses are compared with the distributions obtained from the best possible and all other hypotheses. The distributions for the selected hypotheses approximate the shape of the distributions from the best possible hypotheses reasonably well. As already seen for the reconstruction of the hadronically decaying $W$ bosons the largest differences between the best possible hypothesis, our selected hypothesis and the other hypotheses occur in the reconstructed mass distributions. The distribution for the other hypotheses is again much broader than for the best possible and the selected hypotheses.

\subsection{Data - MC Comparison}

For further validation a comparison between data and Monte Carlo simulation is performed in order to prove that the simulated events reconstructed in the same way as the data describe the data well. For this purpose the background estimation and background samples presented in section 3.4 are used to model the background distributions. For the $t \bar{t}$ signal we use the signal samples presented in section 3.2.2. In order to compare the MC expectation with the observed distributions, the distributions obtained from the MC samples are normalized to the number of observed events in the data.

Figure 4.11 shows the comparison of data and the Monte Carlo prediction for the quantity $\Psi$ as well as for its ingredients $\chi^{2}$ and $P_{\mathrm{b}-\text { light }}$. Since the distributions for $\Psi$ and $P_{\mathrm{b} \text {-light }}$ peak at very small values, the logarithm of both quantities is presented. Overall, reasonable agreement between data and Monte Carlo simulation is obtained. In figure 4.12 the comparison of data and the Monte Carlo simulation for the transverse momentum and the pseudorapidity of the charged lepton, the neutrino, and the leptonically decaying $W$ boson are shown. Figure 4.13 shows the same distributions for the hadronically decaying $W$ boson. In addition, the distribution for the reconstructed mass of the $W$ boson is displayed. The distributions for the two top quarks are presented in figures 4.14 and 4.15. All distributions show no discrepancies between the simulated and measured values within statistical fluctuations and we therefore conclude that a good agreement between data and Monte Carlo simulation is obtained. 
(a)

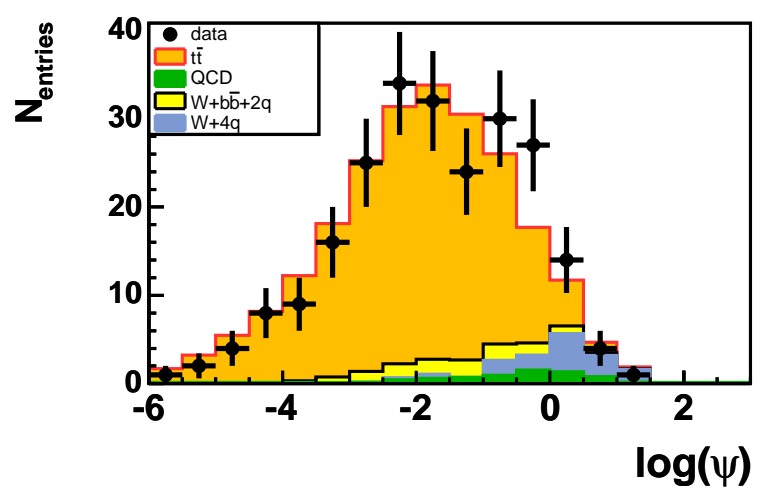

(b)

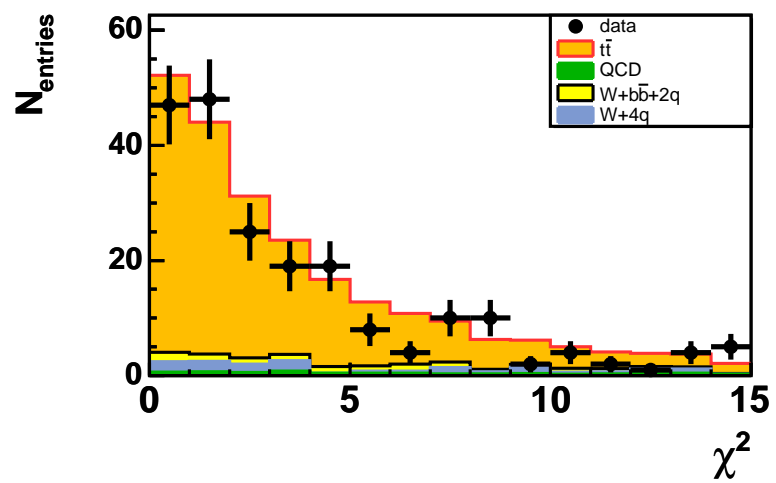

(c)

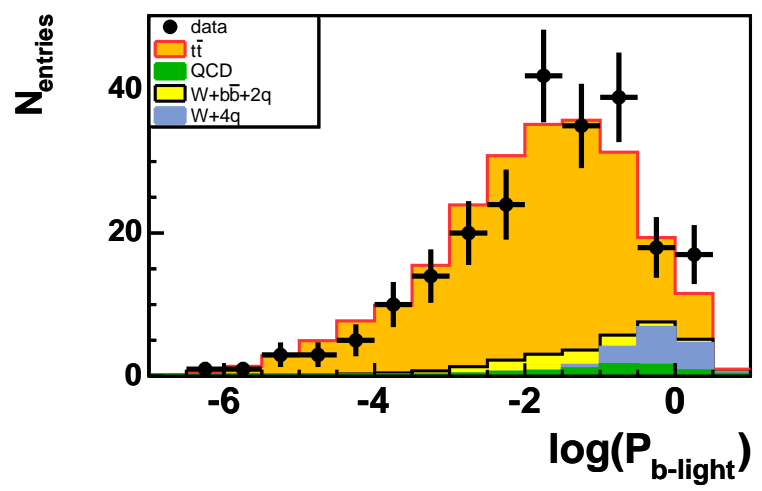

Figure 4.11: Comparison of data and Monte Carlo simulation for (a) the quantity $\Psi$ and its ingredients (b) $\chi^{2}$ and (c) $P_{\mathrm{b}-\text { light }}$. Since the distribution for $\Psi$ as well as for $P_{\mathrm{b}-\text { light peaks at }}$ very small values, we show the distribution of the logarithm of these two quantities. 
(a)

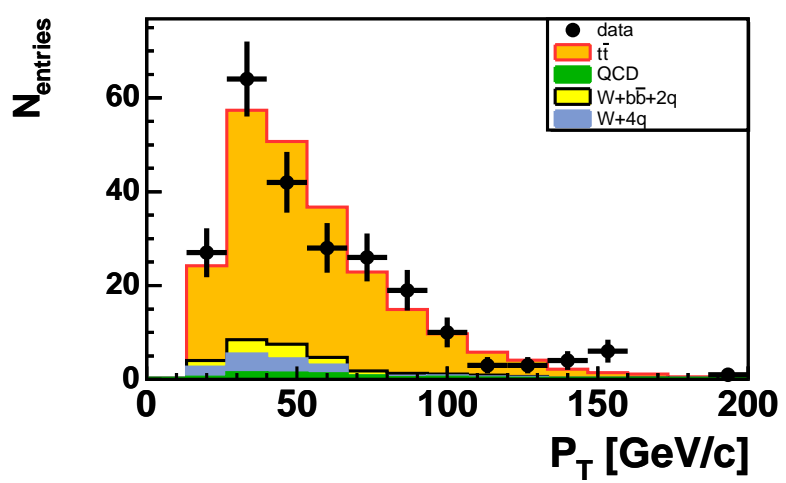

(c)

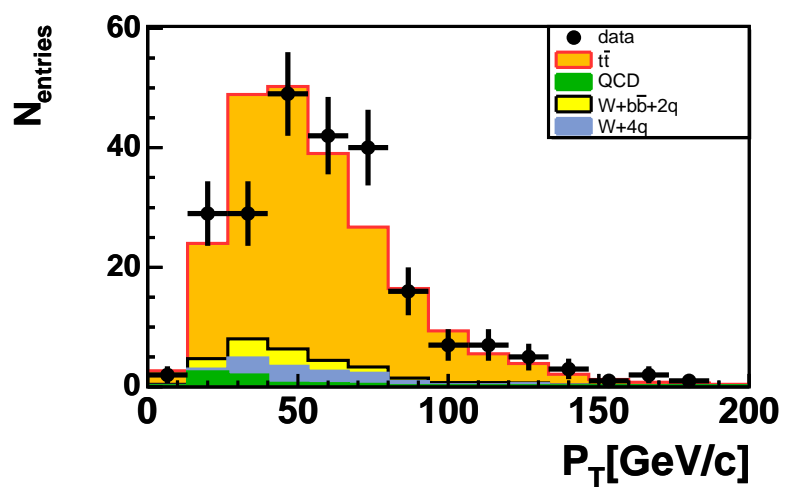

(e)

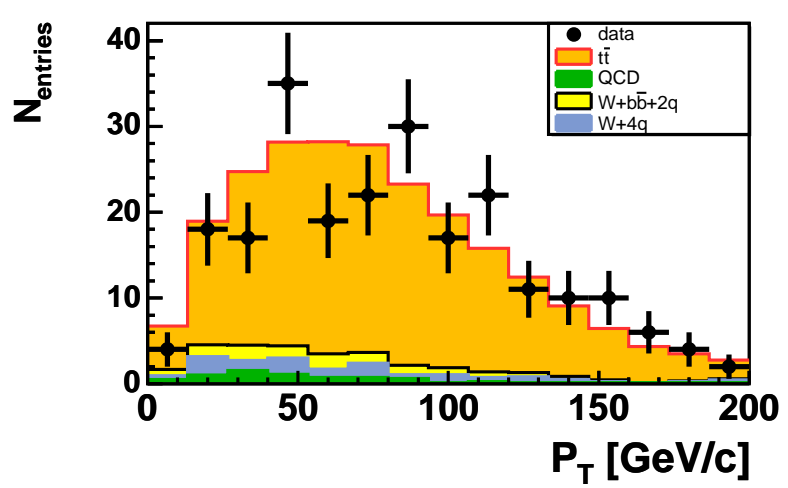

(b)

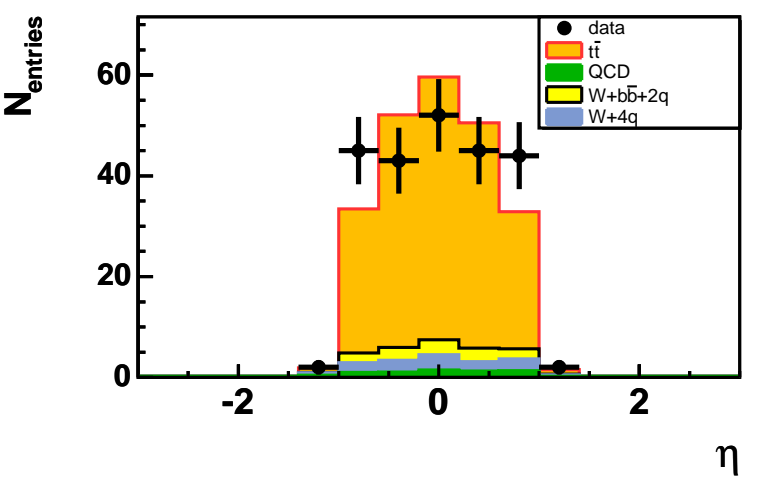

(d)

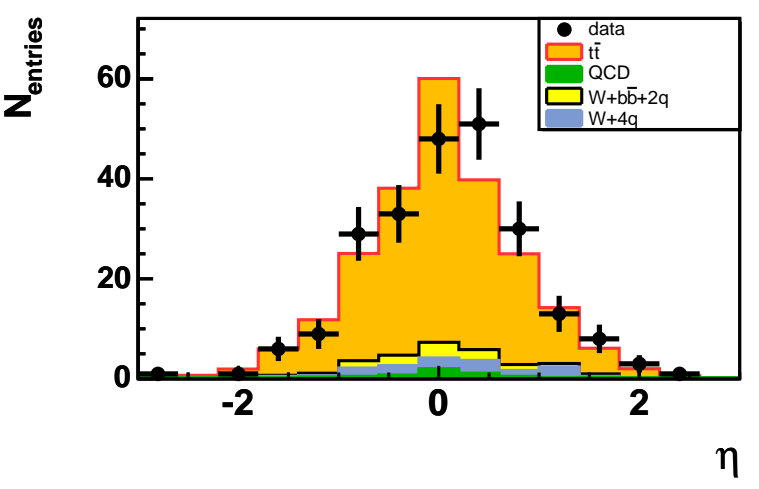

(f)

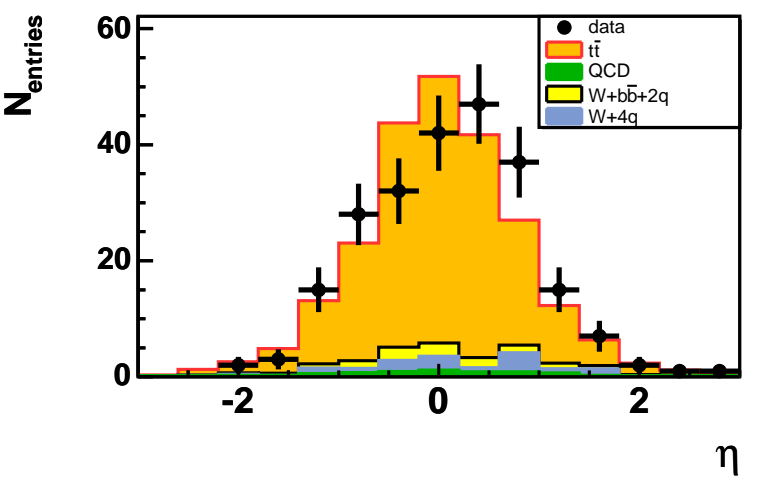

Figure 4.12: Comparison of data and Monte Carlo simulation for the transverse momentum and the pseudorapidity (a)+(b) of the charged lepton, $(c)+(d)$ of the neutrino, and (e)+(f) of the leptonically decaying $\mathbf{W}$ boson. All four plots show no discrepancies between the Monte Carlo prediction and the data within statistical fluctuations. 
(a)

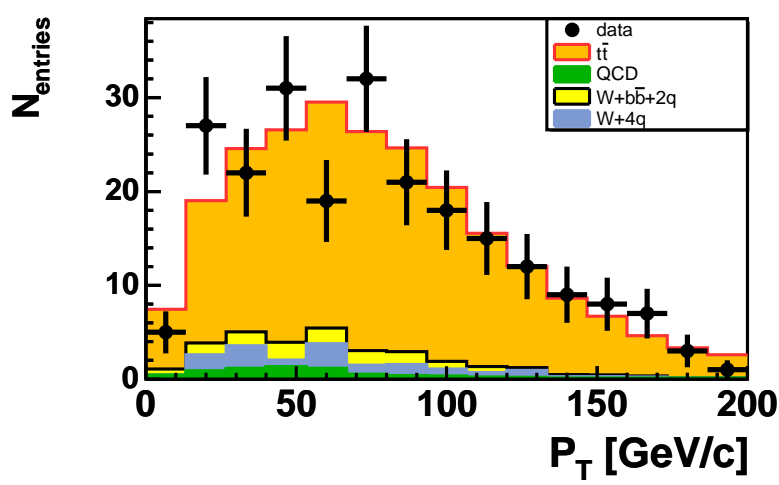

(c)

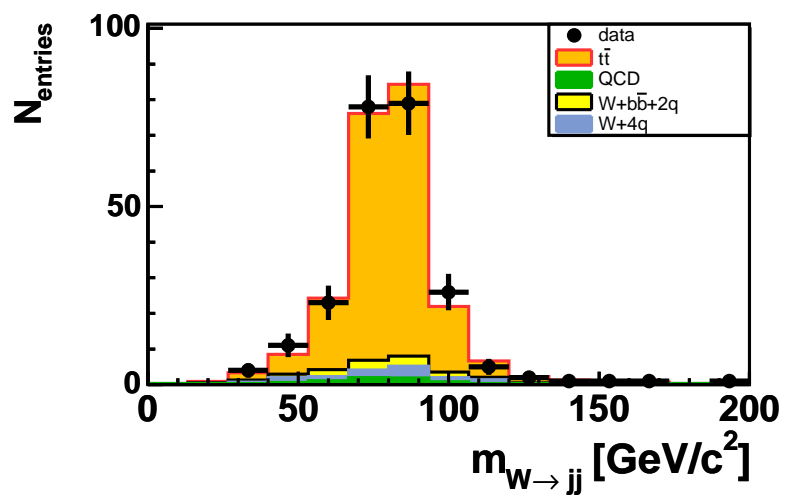

(b)

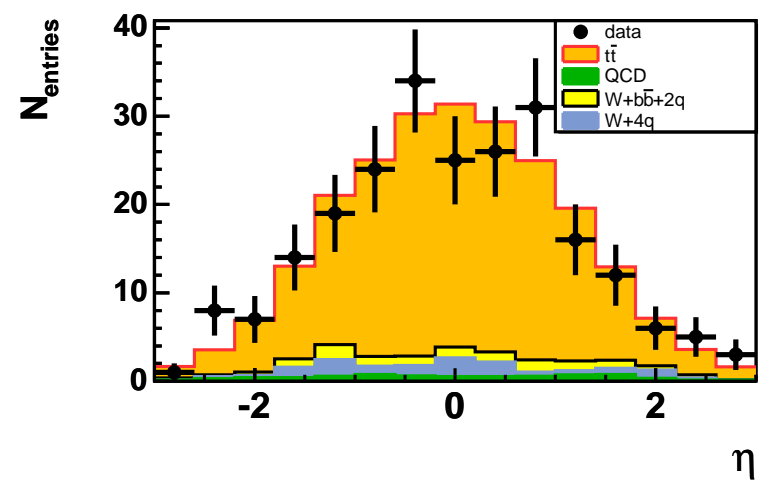

Figure 4.13: Comparison of data and Monte Carlo simulation for (a) the transverse momentum, (b) the pseudorapidity, and (c) the mass of the hadronically decaying $\mathbf{W}$ boson. All three plots show no discrepancies between the Monte Carlo prediction and the data within statistical fluctuations. 
(a)

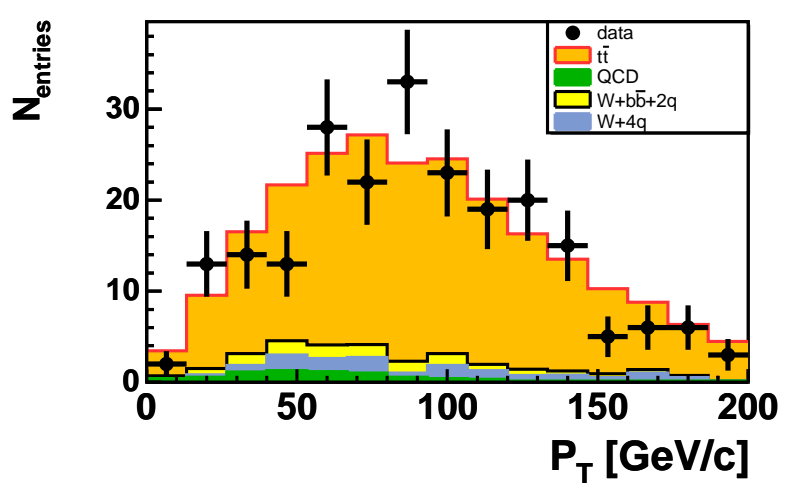

(c)

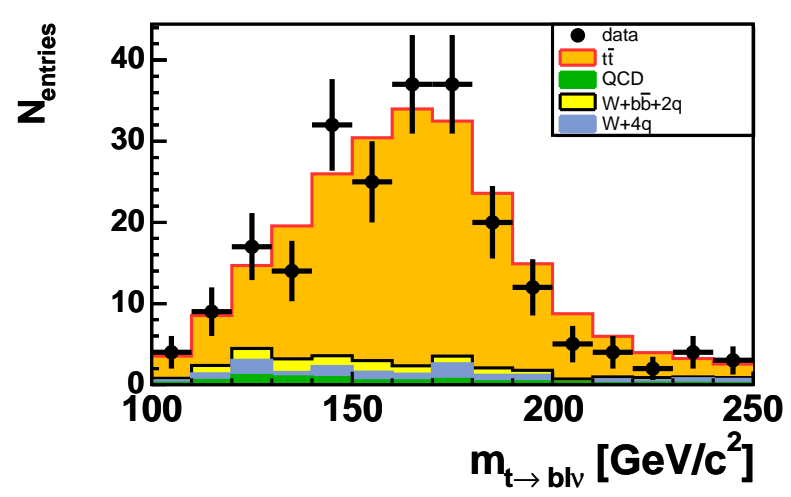

(b)

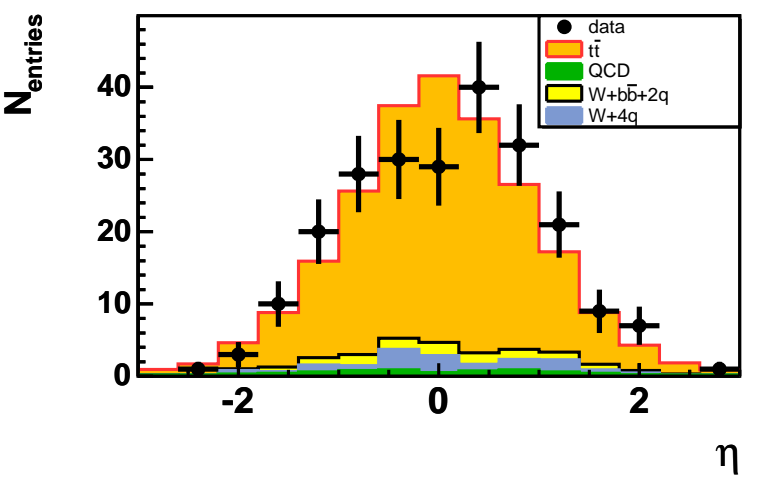

Figure 4.14: Comparison of data and Monte Carlo simulation for (a) the transverse momentum, (b) the pseudorapidity, and (c) the mass of the semileptonically decaying top quark. All three plots show no discrepancies between the Monte Carlo prediction and the data within statistical fluctuations. 
(a)

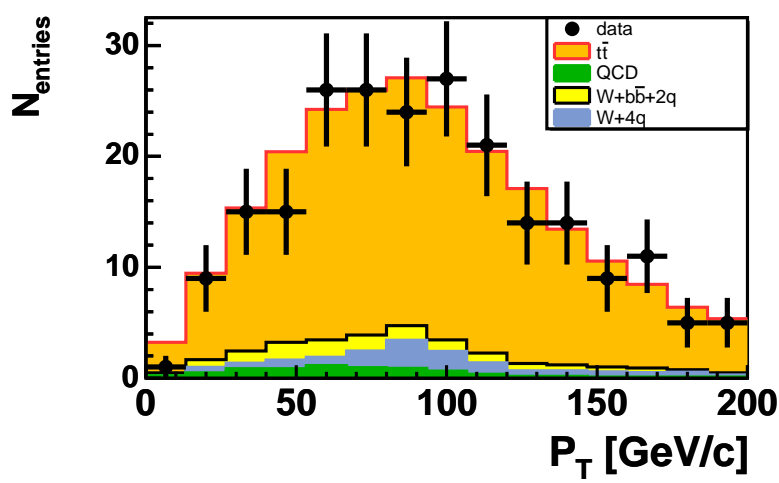

(c)

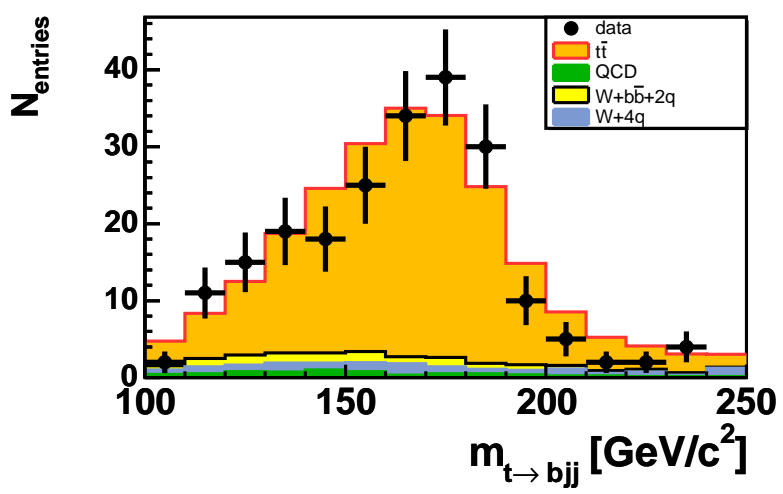

(b)

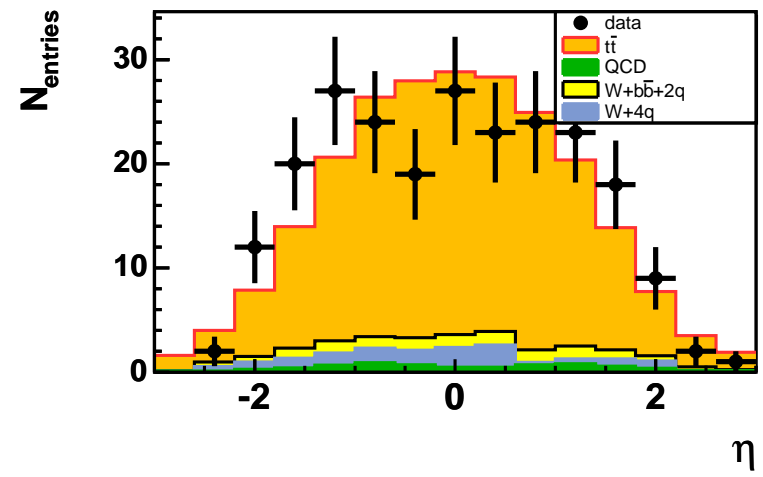

Figure 4.15: Comparison of data and Monte Carlo simulation for (a) the transverse momentum, (b) the pseudorapidity, and (c) the mass of the hadronically decaying top quark. All three plots show no discrepancies between the Monte Carlo prediction and the data within statistical fluctuations. 


\section{Chapter 5}

\section{Measurement of the Helicity Fractions}

In this chapter the method for the measurement of the fraction of longitudinal polarized $W$ bosons $F_{0}$ and the fraction of right handed $W$ bosons $F_{+}$is described. In the first section a brief overview of our method is given, while the single steps of the analysis are then described in the following sections.

\subsection{Measurement Strategy}

As mentioned in section 1.4 as sensitive observable we analyze the cosine of the decay angle $\theta^{*}$ which is defined as the angle between the momentum of the charged lepton in the $W$ boson rest frame and the momentum of the $W$ boson in the top quark rest frame. For illustration figure 5.1 provides a schematic view of that angle.

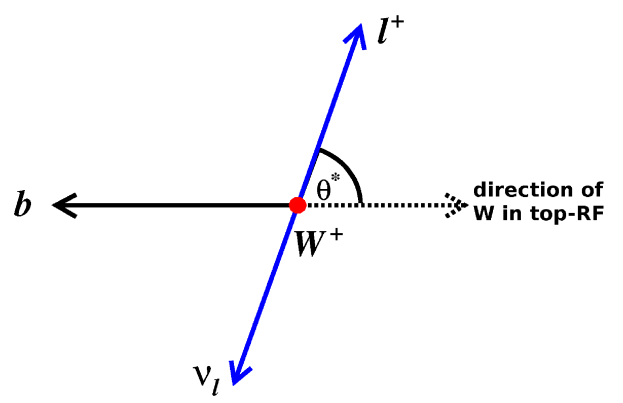

Figure 5.1: Schematic view of the decay angle $\theta^{*}$ in the rest frame of the $W$ boson. The dotted black arrow represents the direction of the $W$ boson in the rest frame (RF) of the top quark.

The distribution of the cosine of this angle is proportional to $\left(1-\cos \theta^{*}\right)^{2}$ for left handed $W$ bosons, proportional to $\left(1+\cos \theta^{*}\right)^{2}$ for right handed $W$ bosons, and proportional to $\left(1-\cos ^{2} \theta^{*}\right)$ for longitudinally polarized $W$ bosons. The $\cos \theta^{*}$ distribution for a certain composition of the three helicity fractions is given by: 
$\frac{d N}{d \cos \theta^{*}}=F_{-} \cdot \frac{3}{8}\left(1-\cos \theta^{*}\right)^{2}+F_{0} \cdot \frac{3}{4}\left(1-\cos ^{2} \theta^{*}\right)+F_{+} \cdot \frac{3}{8}\left(1+\cos \theta^{*}\right)^{2}$.

The theoretical $\cos \theta^{*}$ distributions for the three helicity modes resulting from the equation above are shown in figure 5.2. In addition, also the distribution assuming the SM values of $F_{0}, F_{-}$, and $F_{+}$is plotted.

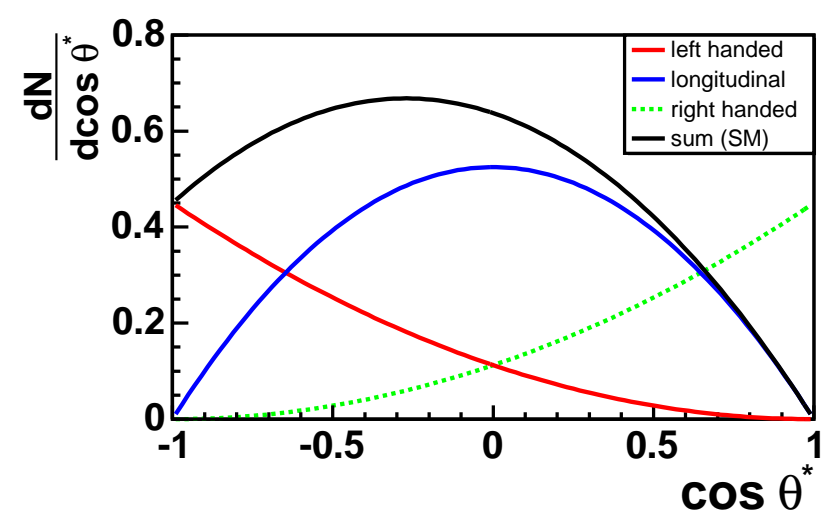

Figure 5.2: Theoretical $\cos \theta^{*}$ distribution for left handed (red), right handed (green dashed) and longitudinally polarized (blue) $W$ bosons. The distribution predicted by the SM is indicated by the black solid line.

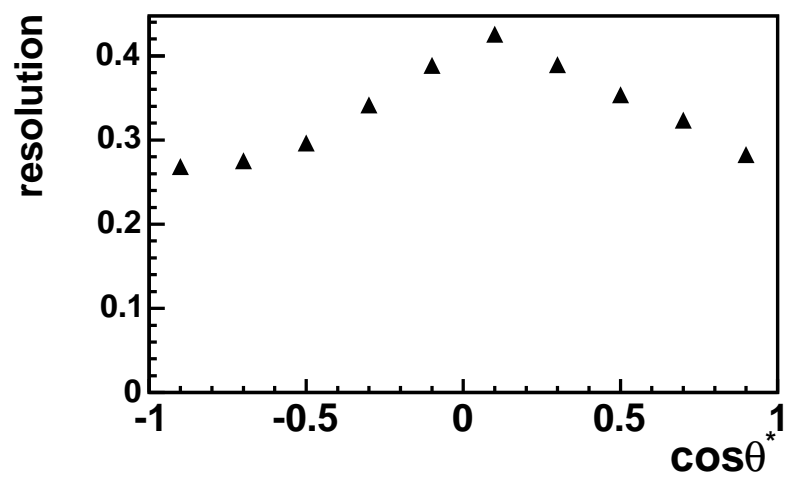

Figure 5.3: Resolution of the full reconstruction in bins of the $\cos \theta^{*}$ distribution. The single values represent the Gaussian widths of the $\left(\cos \theta_{\text {gen }}^{*}-\cos \theta_{\text {rec }}^{*}\right)-$ distributions in each bin of the $\cos \theta^{*}$ distribution obtained from a Pүтніа MC sample.

To deduce a proper value for the number of bins for the observed $\cos \theta^{*}$ distribution, we determine the resolution of the full reconstruction using a $\mathrm{MC}$ sample generated with PyтhiA. For ten bins of the $\cos \theta^{*}$ distribution a Gaussian is fitted 
to the distribution of the difference between the $\cos \theta^{*}$ values obtained from generated and reconstructed four-vectors $\left(\cos \theta_{\text {gen }}^{*}-\cos \theta_{\text {rec }}^{*}\right)$. The widths of these ten Gaussians are shown in figure 5.3. Since the resolution of the full $t \bar{t}$ reconstruction takes values between 0.2 and 0.4 we decide to use five bins for the observed $\cos \theta^{*}$ distribution.

Figure 5.4 shows the $\cos \theta^{*}$ distribution for the 232 fully reconstructed $t \bar{t}$ event candidates in data. In (a) the observed distribution (black points) is shown together with the SM expectation (red line) obtained from MC samples and the uncertainty on that expectation (yellow band). We also show a plot (b) where the different contributions of background and signal are stacked on top of each other. In both cases the distribution obtained from MC samples is normalized to the number of observed events.

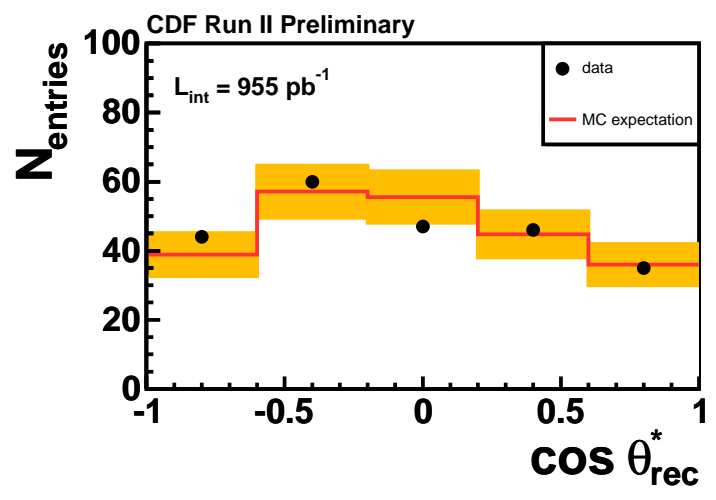

(a)

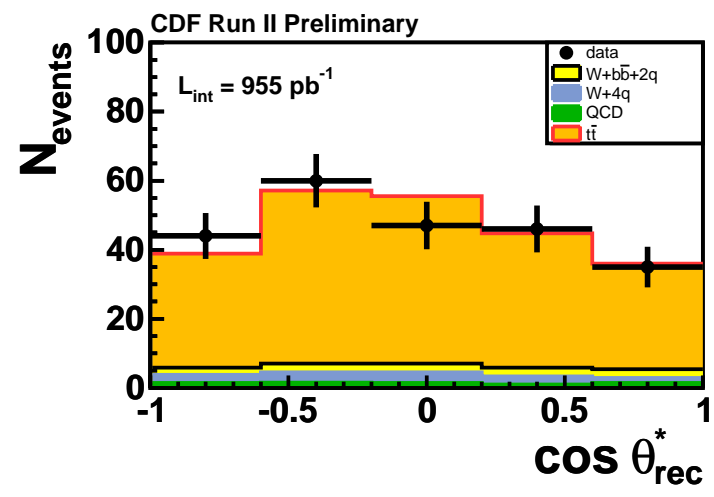

(b)

Figure 5.4: Reconstructed $\cos \theta_{\text {rec }}^{*}$ distribution. In (a) the observed distribution (black points) is shown together with the SM expectation obtained from MC samples. The yellow band gives the uncertainty on the expectation. In (b) the different expected contributions of background and signal are stacked on top of each other.

Comparing the shapes of the calculated $\cos \theta^{*}$ distribution for SM helicity fractions in figure 5.2 and of the $\cos \theta_{\text {rec }}^{*}$ distribution obtained from reconstructed $\mathrm{MC}$ events, generated with SM PYтніA, in figure 5.4, it is noticeable that they exhibit some differences. That is understandable, since the true distribution is influenced by detector mismeasurements, selection and reconstruction effects.

Thus, the reconstructed distribution cannot directly be compared to the calculated distributions and in order to extract the helicity fractions from the reconstructed $\cos \theta_{\text {rec }}^{*}$ distribution the theoretical distributions for the three helicity modes cannot be utilized as fit templates but have to be corrected by taking the occurring effects into account. How these effects enter the calculation of our fit templates is described in the next section. In order to measure the helicity fractions, a binned 
likelihood fit using the calculated fit templates is performed.

After the fit, the reconstructed distribution is unfolded offering the possibility for direct comparison between data and the theoretical distribution presented in figure 5.2. For that purpose a transfer function corresponding to the measured helicity fraction is constructed. The single steps of our analysis method are now described in more detail.

\subsection{Calculation of the normalized Signal Templates}

The fractions of right handed and longitudinally polarized $W$ bosons are measured performing a binned likelihood fit. Therefor we need the expected number of events in each bin, dependent on the values of $F_{0}$ and $F_{+}$. In this section the calculation of the normalized signal templates $\hat{\mu}_{i}^{\text {sig,obs }}\left(F_{0}, F_{+}\right)$for each bin $i$ is described. The number of expected signal events in a certain bin $\mu_{i}^{\mathrm{sig}, \mathrm{obs}}\left(F_{0}, F_{+}\right)$is then obtained by multiplying the normalized signal template with the total number of expected signal events: $\mu_{i}^{\text {sig,obs }}\left(F_{0}, F_{+}\right)=\hat{\mu}_{i}^{\text {sig,obs }}\left(F_{0}, F_{+}\right) \cdot N_{\text {sig. }}$. The index "sig,obs" denotes the fact that $\mu_{i}$ is the number of signal events that are expected to be observed in the data after applying all selection cuts and performing the full event reconstruction.

The starting point of the calculation of the normalized signal template $\hat{\mu}_{i}^{\text {sig,obs }}$ is the contribution of $t \bar{t}$ signal events $\hat{\mu}_{i}^{\text {sig }}$ in the $i^{\text {th }}$ bin of $\cos \theta^{*}$ before applying any cuts. This distribution is obtained by integrating equation 5.1 for each bin separately and using the relation $F_{0}+F_{-}+F_{+}=1$ to eliminate the fraction of left handed $W$ bosons:

$$
\hat{\mu}_{i}^{\text {sig }}\left(F_{0}, F_{+}\right)=\left(1-F_{0}-F_{+}\right) \cdot f_{i}^{-}+F_{0} \cdot f_{i}^{0}+F_{+} \cdot f_{i}^{+}
$$

where $f_{i}^{-}, f_{i}^{+}$and $f_{i}^{0}$ are the fractions of events contained in bin $i$ in case of left handed, right handed, and longitudinally polarized $W$ bosons:

$$
\begin{aligned}
f_{i}^{0} & =\int_{a_{i}}^{b_{i}} \frac{3}{4}\left(1-\cos ^{2} \theta^{*}\right) d \cos \theta^{*}, \\
f_{i}^{-} & =\int_{a_{i}}^{b_{i}} \frac{3}{8}\left(1-\cos \theta^{*}\right)^{2} d \cos \theta^{*}, \\
f_{i}^{+} & =\int_{a_{i}}^{b_{i}} \frac{3}{8}\left(1+\cos \theta^{*}\right)^{2} d \cos \theta^{*} .
\end{aligned}
$$

The fractions $f^{0}, f^{-}$, and $f^{+}$are normalized to unit area, hence the relations $\sum_{i}^{N_{\text {bins }}} f_{i}^{0}=1, \sum_{i}^{N_{\text {bins }}} f_{i}^{-}=1$, and $\sum_{i}^{N_{\text {bins }}} f_{i}^{+}=1$ hold. $a_{i}$ and $b_{i}$ denote the lower and upper edge of bin $i$ and $N_{\text {bins }}$ is the number of bins. Rewriting equation (5.2) points out the linear dependence of $\hat{\mu}_{i}^{\text {sig }}$ on $F_{0}$ and on $F_{+}$:

$$
\begin{aligned}
\hat{\mu}_{i}^{\mathrm{sig}}\left(F_{0}, F_{+}\right) & =F_{0} \cdot\left[f_{i}^{0}-f_{i}^{-}\right]+F_{+}\left[f_{i}^{+}-f_{i}^{-}\right]+f_{i}^{-} \\
& =F_{0} \cdot A_{i}+F_{+} \cdot B_{i}+C_{i} .
\end{aligned}
$$


Here the coefficients $A_{i}, B_{i}$ and $C_{i}$ of the $i^{\text {th }}$ bin are defined as:

$$
\begin{aligned}
A_{i} & =f_{i}^{0}-f_{i}^{-}, \\
B_{i} & =f_{i}^{+}-f_{i}^{-}, \\
C_{i} & =f_{i}^{-} .
\end{aligned}
$$

The effects that have to be considered calculating the observed normalized signal templates $\hat{\mu}_{i}^{\text {sig,obs }}$ out of the theoretical normalized signal templates $\hat{\mu}_{i}^{\text {sig }}$ can be divided into two types. One impact on the $\cos \theta^{*}$ distribution is due to the event selection, while the second kind of effects arises from detector mismeasurement and the fact that the full reconstruction is not perfectly efficient. The impact of both kinds of effects on the true $\cos \theta^{*}$ distribution obtained by MC studies is displayed in figure 5.5. In order to place emphasis on the impact on the shape of the $\cos \theta^{*}$ distribution the distribution for generated events is normalized to the number of selected events. In the following the influence of both effects on the $\cos \theta^{*}$ distribution and how each effect is considered in the calculation of the normalized signal templates is described in more detail.

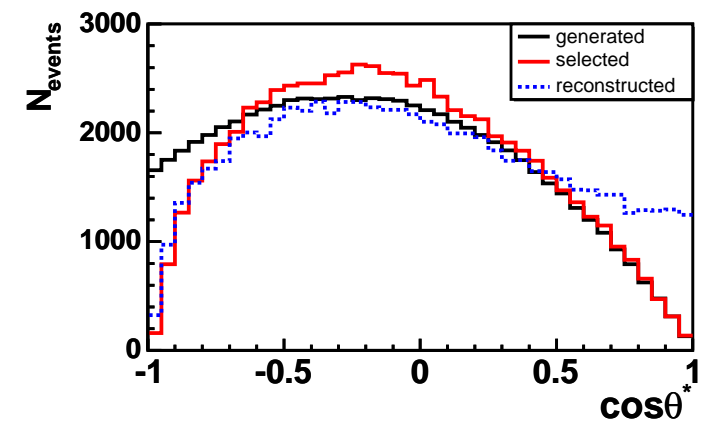

(a)

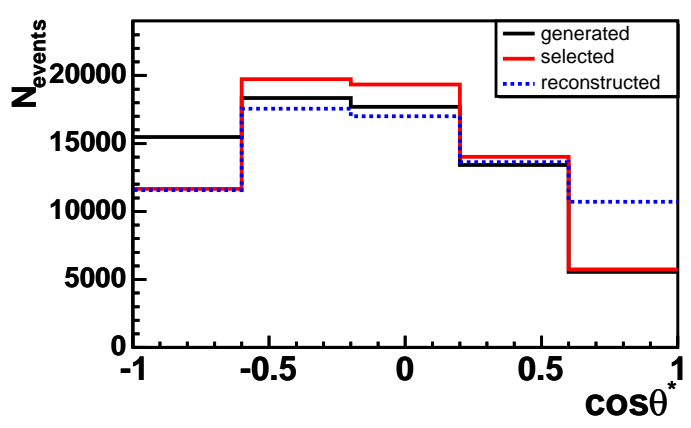

(b)

Figure 5.5: Impact of event selection and reconstruction on the $\cos \theta^{*}$ distribution obtained from a MC sample generated with PүтніA. The black solid line represents the distribution before applying any selection cuts, the distribution for the selected events is shown in red. Both distributions are obtained from the generated four-vectors. The blue dashed line represents the distribution obtained from fully reconstructed events (for each event the hypothesis with the smallest value of $\Psi$ is chosen). The distributions are normalized to the number of selected events and are presented in (a) 40 bins as well as in (b) 5 bins.

\section{Impact of the Event Selection}

Events with values for $\cos \theta^{*}$ close to -1 are suppressed, since in these events the charged lepton flies in the same direction as the $b$ quark (see figure 5.1) and therefore they are much stronger rejected by the isolation requirement than events with 
different values for $\cos \theta^{*}$. The isolation requirement rejects events in which the nonlepton $E_{\mathrm{T}}$ in a cone in the $\eta$ - $\phi$ plane of radius 0.4 centered around the lepton is more than $10 \%$ of the lepton $E_{\mathrm{T}}$. The second reason for the strong rejection of events with values for $\cos \theta^{*}$ close to -1 is due to the softer $p_{\mathrm{T}}$ spectrum of leptons being emitted in backward direction with respect to the $W$ boson direction of motion. Thus, these events are more likely to fail the lepton $p_{\mathrm{T}}$ cut in the event selection than events with larger values for $\cos \theta^{*}$. For events with values for $\cos \theta^{*}$ close to +1 this effect is compensated by the fact that $\mathbb{E}_{\mathrm{T}}$ decreases with increasing values for $\cos \theta^{*}$. In these events the neutrino is emitted in backward direction with respect to the $W$ boson direction and therefore its transverse energy is smaller than in the opposite case. Thus, for large positive values of $\cos \theta^{*}$ the events are more likely to pass the lepton $p_{\mathrm{T}}$ requirement, but also more likely to fail the $\mathbb{E}_{\mathrm{T}}$ cut. Figure 5.5 shows the $\cos \theta^{*}$ distributions for all generated events and for the selected events. Both distributions are obtained from the generated four-vectors. The variation in the shape of the $\cos \theta^{*}$ distribution is thus a consequence of the $\cos \theta^{*}$ dependence of the event selection efficiency. Consequently this effect is taken into account by applying different efficiencies for different bins of the $\cos \theta^{*}$ distribution to calculate the number of selected events for each bin.

May $N$ be the total number of generated events, then $N_{i}=N \cdot \hat{\mu}_{i}^{\text {sig }}$ is the number of events in bin $i$ of the $\cos \theta^{*}$ distribution before applying any selection cuts. Considering the dependence of the efficiency on $\cos \theta^{*}$ the number of selected events in a certain bin is thus given by:

$$
N_{i}^{\mathrm{sel}}=N_{i} \cdot \frac{N_{i}^{\mathrm{sel}}}{N_{i}}=N_{i} \cdot \epsilon_{i}^{\mathrm{abs}}
$$

From the trivial definition of the number of selected events, $N_{i}^{\text {sel }}=N_{i} \cdot\left(N_{i}^{\text {sel }} / N_{i}\right)$, the absolute efficiency is defined as the fraction of the number of selected events in a certain bin of $\cos \theta^{*}$ with respect to the number of generated events in that bin: $\epsilon_{i}^{\mathrm{abs}}=N_{i}^{\mathrm{sel}} / N_{i}$.

We assume, that the shape of the distribution of the absolute efficiency as a function of $\cos \theta^{*}$ is independent on the helicity of the $W$ bosons, which is equivalent to the assumption that the only difference of left handed, right handed, and longitudinally polarized $W$ bosons is due to the different $\cos \theta^{*}$ distribution. All other differences between the different helicities, such as different $p_{\mathrm{T}}$ distributions and different total event acceptances, are caused by the different $\cos \theta^{*}$ distributions.

This assumption is studied in figure 5.6 using normalized efficiencies $\epsilon_{i}^{\text {norm }}=$ $\epsilon_{i}^{\text {abs }} / \sum_{i} \epsilon_{i}^{\text {abs }}$ which allow direct comparison between the shapes of efficiency distributions for different settings of helicity fractions. Since the aim of this section is to calculate normalized signal templates, only the shape of the $\epsilon_{i}^{\text {abs }}$ distribution matters. In other words, only the relations between the efficiencies in different bins and not the absolute values of the efficiencies in certain bins are of interest. Therefore, we can multiply $\epsilon_{i}^{\text {abs }}$ with an arbitrary factor that cancels later out due to 


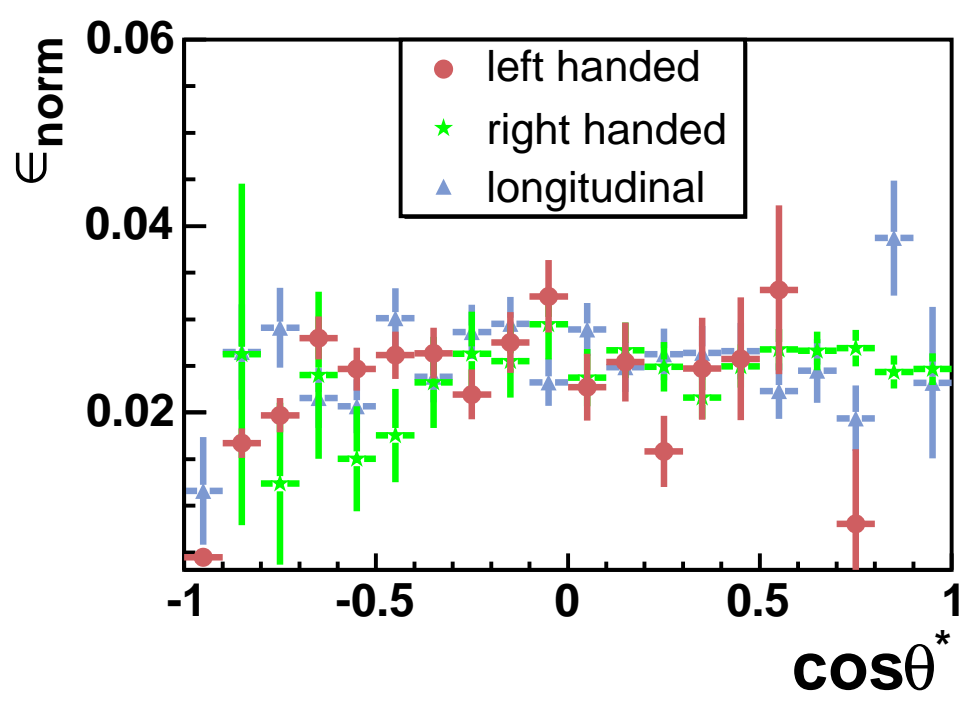

Figure 5.6: Normalized efficiency $\epsilon_{i}^{\text {abs }} / \sum_{i} \epsilon_{i}^{\text {abs }}$ as a function of $\cos \theta^{*}$ for left handed, right handed and longitudinally polarized $W$ bosons determined with the GGWIG samples.

the normalization. For simplicity we utilize relative efficiencies instead of absolute efficiencies. The relative efficiency $\epsilon_{i}^{\text {rel }}$ is defined as:

$$
\epsilon_{i}^{\mathrm{rel}}=\frac{N_{i}^{\mathrm{sel}} / N^{\mathrm{sel}}}{N_{i} / N}
$$

and is obtained from $\epsilon_{i}^{\text {abs }}$ by dividing by the acceptance factor $\mathcal{A}=N^{\text {sel }} / N$ :

$$
\epsilon_{i}^{\mathrm{rel}}=\frac{\epsilon_{i}^{\mathrm{abs}}}{\mathcal{A}} .
$$

Substituting the absolute efficiency with the relative efficiency $\epsilon^{\text {rel }}$ the expected number of events in bin $i$ after applying all selection cuts is defined as:

$$
N_{i}^{\mathrm{sel}}=N_{i} \cdot \epsilon_{i}^{\mathrm{rel}} \cdot \mathcal{A} .
$$

\section{Impact of the Event Reconstruction}

Apart from the influence of the event selection also the event reconstruction has an impact on the $\cos \theta^{*}$ distribution. Several effects can be merged under the label "reconstruction effects". 
As mentioned in chapter 4, several ambiguities occur during the reconstruction of a $t \bar{t}$ event candidate which lead to numerous event interpretations. One impact on the $\cos \theta^{*}$ distribution is due to the fact that only in $34 \%$ of all events the best possible event interpretation is chosen. Hence in several cases we choose the wrong combination of jets and leptons to reconstruct the top quarks and $W$ bosons and therefore the value for $\cos \theta^{*}$ for the reconstructed events differs from the true value calculated from the generated four-vectors.

But even if we choose the best possible hypothesis, the value for $\cos \theta^{*}$ obtained from the reconstructed four-vectors can vary significantly from the value calculated from the generated four-vectors. In nearly all events the best possible event hypothesis itself has a small deviation from the MC true values, for some events that deviation is rather large (see figure 4.6).

One reason for this deviation lies in the radiation of soft gluons. Since these low-energy gluons do not fulfill all the jet requirements, they are not selected as jets. As a consequence, the energy of the jet radiating a gluon is decreased and does not reflect the energy of the original quark from the top quark decay. This effect is considered by the out-of-cone jet corrections but is not totally compensated in all events. A further contribution to that deviation arises from the finite resolution of the energy and direction measurement of jets and particles itself. The most sensitive quantity is here the missing transverse energy. Another contribution to this deviation arises from the fact that in some events not all four jets originating from the top quark decay are selected but due to additional selected jets from hard gluon radiation the event is accepted. In this case it is impossible to reconstruct the $t \bar{t}$ pair correctly since not all components are available.

Besides these effects, either arising from wrong combination of the measured values, from the fact that not all components are available, or from mismeasurements in the detector, also a more fundamental problem exists. Quarks from the top quark decay hadronize and form particle jets. In the hadronization process color flux tubes are stretched between the quarks produced in the top quark decay. The potential energy stored in these strings increases as the quarks move apart and the strings may break by the production of new quark-antiquark pairs. This fragmentation leads to the formation of colorless particles which build up particle jets. Though in most cases the jets follow the direction of the quarks they originate from, this is not ensured. The direction of the measured jet thus does not give exactly the direction of the final state partons in all events, and therefore in some events it is impossible to reconstruct correctly the four-vectors of the two top quarks from the detected objects.

All the mentioned effects have an influence on the $\cos \theta_{\text {rec }}^{*}$ distribution obtained from the reconstructed four-vectors. Thus, events with a generated value for $\cos \theta^{*}$ corresponding to a certain bin of the $\cos \theta^{*}$ distribution can occur in a different bin after the event reconstruction. This impact on the $\cos \theta^{*}$ distribution is called 

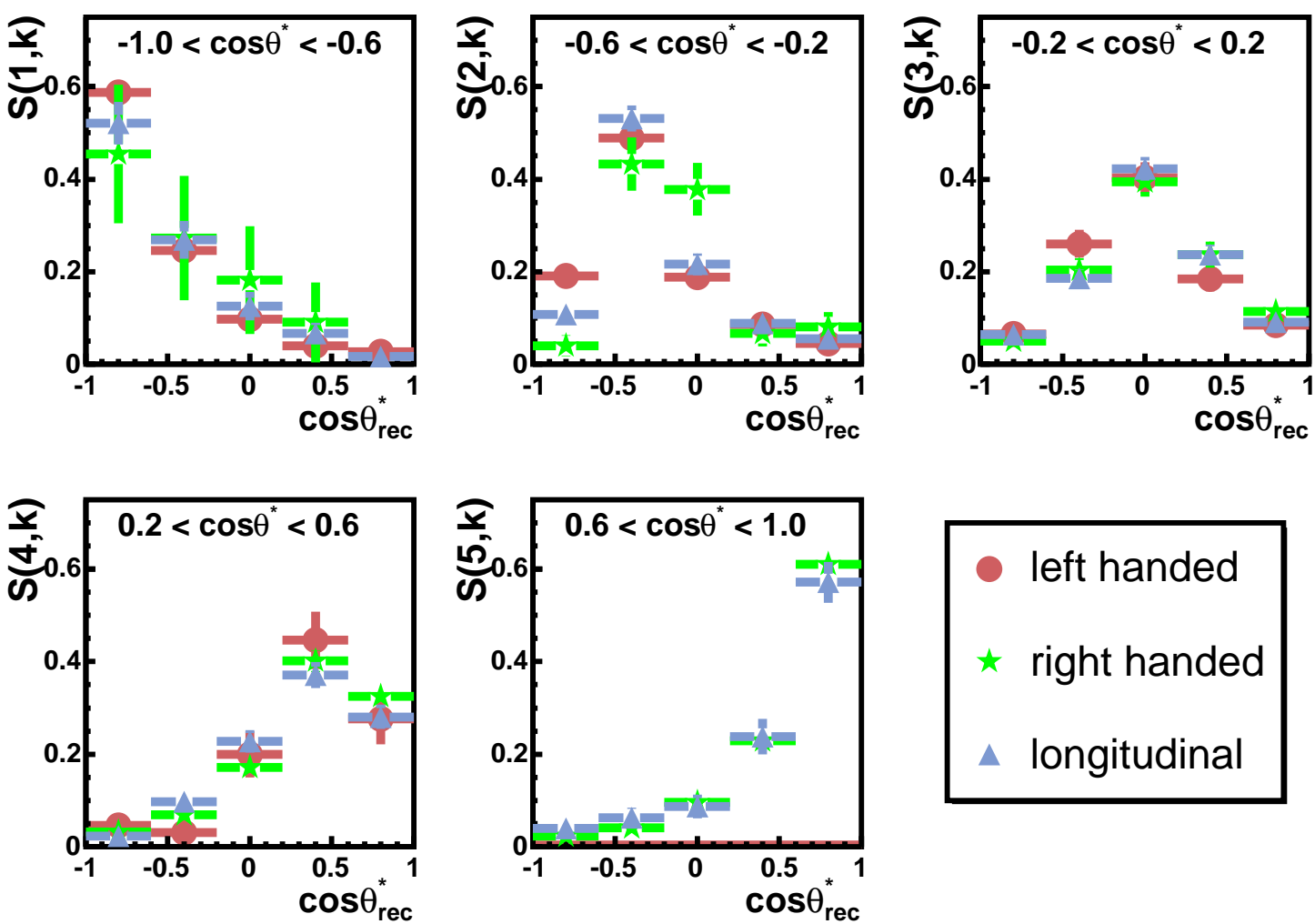

\section{- left handed \\ * right handed \\ $\Delta$ longitudinal}

Figure 5.7: Migration matrix element $S(i, k)$ as a function of $\cos \theta_{\text {rec }}^{*}$ in five bins of $\cos \theta^{*}$ for left handed, right handed, and longitudinally polarized $W$ bosons determined with the GGWIG samples. 

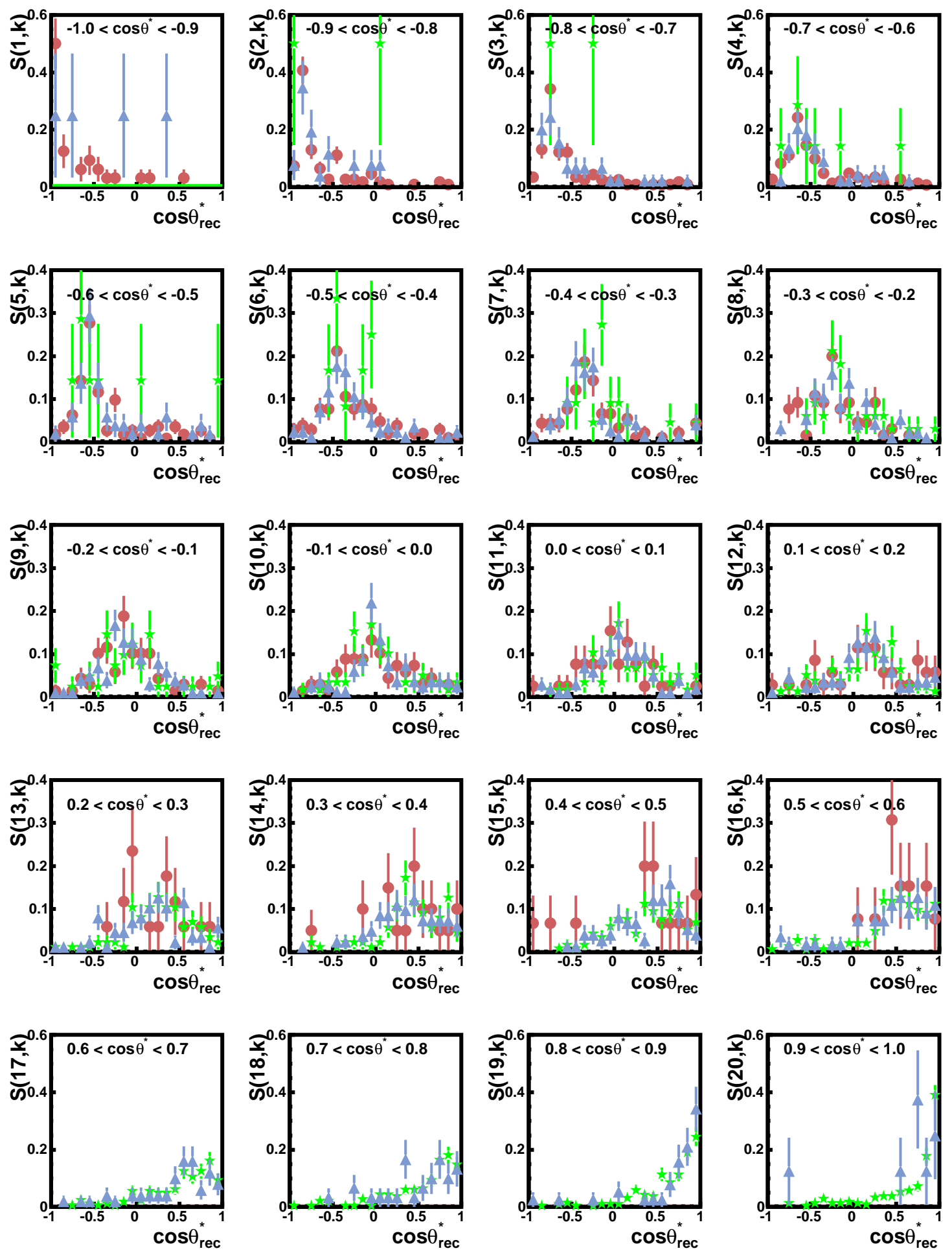

Figure 5.8: Migration matrix element $S(i, k)$ as a function of $\cos \theta_{\text {rec }}^{*}$ in 20 bins of $\cos \theta^{*}$ for left handed, right handed, and longitudinally polarized $W$ bosons determined with the GGWIG samples. 
"migration" and is taken into account by applying the migration matrix $S$. The matrix element $S(i, k)$ gives the probability for an event with a true value for $\cos \theta^{*}$ in bin $i$ to occur after the reconstruction in bin $k$ of the $\cos \theta_{\text {rec }}^{*}$ distribution. Since all events of bin $i$ have to occur somewhere in the reconstructed $\cos \theta_{\text {rec }}^{*}$ distribution, the matrix is defined in such a way, that $\sum_{k} S(i, k)=1$ holds for all bins $i$.

The expected number of events in bin $k$ after event selection and reconstruction is thus given by the sum over the numbers of selected events in every bin multiplied with the corresponding probability to be reconstructed in bin $k$ :

$$
N_{k}^{\mathrm{rec}}=\sum_{i} N_{i}^{\mathrm{sel}} \cdot S(i, k)
$$

We assume that like the efficiency also the migration matrix $S$ depends neither on $F_{0}$ nor on $F_{+}$. This assumption is checked in figure 5.7 and 5.8, where the migration matrix element $S(i, k)$ as a function of the reconstructed $\cos \theta_{\text {rec }}^{*}$ values in five bins and 20 bins respectively of the true $\cos \theta^{*}$ distribution is shown. Due to the low statistics of the GGWIG sample only five bins are used when the GGWIG sample is used for further cross checks. For the $20 \times 20$ migration matrix the matrix elements are in good agreement for the three differently polarized $W$ bosons. For the $5 \times 5$ migration matrix, which is not used for the real measurement, some smaller deviations due to different $\cos \theta^{*}$ shapes of the differently polarized $W$ bosons within the large bins are observed.

\section{Normalized Signal Templates}

The normalized signal template $\hat{\mu}_{k}^{\text {sig,obs }}$ which is defined as the fraction of the expected number of reconstructed events in bin $k$ of the $\cos \theta_{\text {rec }}$ distribution with respect to the total number of reconstructed events can now be calculated. Since every selected event is also reconstructed the total number of reconstructed events is equal to the total number of selected events.

$$
\hat{\mu}_{k}^{\text {sig,obs }}\left(F_{0}, F_{+}\right)=\frac{N_{k}^{\text {rec }}}{N_{\text {total }}^{\text {rec }}}=\frac{\sum_{i} N_{i}^{\text {sel }} \cdot S(i, k)}{\sum_{k} \sum_{i} N_{i}^{\text {sel }} \cdot S(i, k)}
$$

Using the expression for the number of selected events given in equation 5.13 this can be rewritten as:

$$
\begin{aligned}
\hat{\mu}_{k}^{\mathrm{sig}, \mathrm{obs}}\left(F_{0}, F_{+}\right) & =\frac{\sum_{i} N_{i} \cdot \epsilon_{i}^{\mathrm{rel}} \cdot \mathcal{A} \cdot S(i, k)}{\sum_{k} \sum_{i} N_{i} \cdot \epsilon_{i}^{\mathrm{rel}} \cdot \mathcal{A} \cdot S(i, k)} \\
& =\frac{\sum_{i} N_{i} \cdot \epsilon_{i}^{\mathrm{rel}} \cdot S(i, k)}{\sum_{k} \sum_{i} N_{i} \cdot \epsilon_{i}^{\mathrm{rel}} \cdot S(i, k)} .
\end{aligned}
$$

In the last step the decision to use relative efficiencies is vindicated, since the acceptance factor $\mathcal{A}$ occurs in the numerator as well as in the denominator and 
therefore cancels out. Using $N_{i}=N \cdot \hat{\mu}_{i}^{\mathrm{sig}}\left(F_{0}, F_{+}\right)$and $\hat{\mu}_{i}^{\mathrm{sig}}\left(F_{0}, F_{+}\right)=F_{0} \cdot A_{i}+F_{+}$. $B_{i}+C_{i}$ from equation 5.6 the above equation can be transformed to:

$$
\hat{\mu}_{k}^{\mathrm{sig}, \mathrm{obs}}\left(F_{0}, F_{+}\right)=\frac{\sum_{i}\left(F_{0} \cdot A_{i}+F_{+} \cdot B_{i}+C_{i}\right) \cdot \epsilon_{i}^{\mathrm{rel}} \cdot S(i, k)}{\sum_{k} \sum_{i}\left(F_{0} \cdot A_{i}+F_{+} \cdot B_{i}+C_{i}\right) \cdot \epsilon_{i}^{\mathrm{rel}} \cdot S(i, k)}
$$

in which the total number of generated events $N$ cancels out. Defining coefficients $A_{k}^{\text {obs }}, B_{k}^{\text {obs }}$ and $C_{k}^{\text {obs }}$ of the $k^{\text {th }}$ bin of $\cos \theta_{\text {rec }}^{*}$ :

$$
\begin{aligned}
A_{k}^{\mathrm{obs}} & =\sum_{i} A_{i} \cdot \epsilon_{i}^{\mathrm{rel}} \cdot S(i, k), \\
B_{k}^{\mathrm{obs}} & =\sum_{i} B_{i} \cdot \epsilon_{i}^{\mathrm{rel}} \cdot S(i, k), \\
C_{k}^{\mathrm{obs}} & =\sum_{i} C_{i} \cdot \epsilon_{i}^{\mathrm{rel}} \cdot S(i, k),
\end{aligned}
$$

equation (5.17) yields:

$$
\hat{\mu}_{k}^{\text {sig,obs }}\left(F_{0}, F_{+}\right)=\frac{F_{0} \cdot A_{k}+F_{+} \cdot B_{k}+C_{k}}{\sum_{k} F_{0} \cdot A_{k}+F_{+} \cdot B_{k}+C_{k}} .
$$

For the calculation of the signal templates used later in the fit, the efficiency and migration matrix have to be obtained from MC samples. Since neither the efficiency nor the migration matrix are dependent on the $W$ helicity fractions, for this purpose a SM $t \bar{t}$ signal sample generated with PYTHIA is used. The efficiencies and migration matrix elements obtained from this sample are presented in figures 5.9 and 5.10.

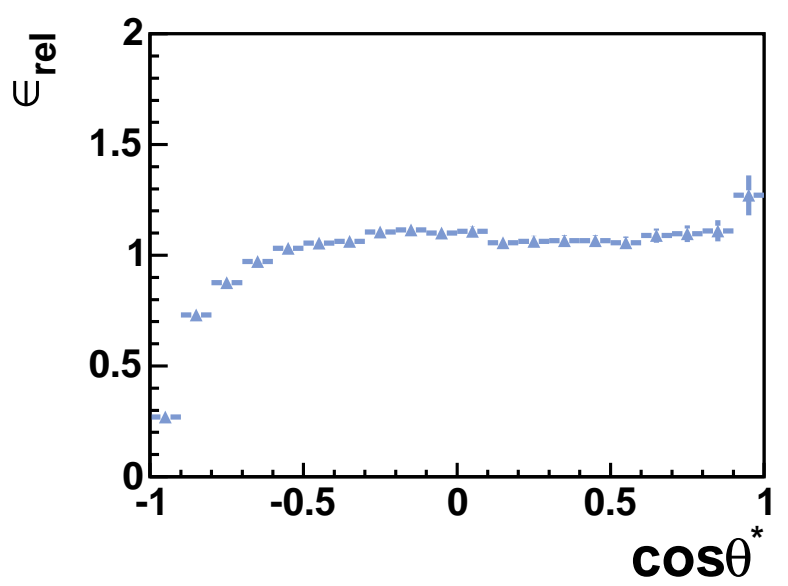

Figure 5.9: Relative efficiency as a function of $\cos \theta^{*} \operatorname{computed}$ with the SM Monte Carlo PYTHIA

The efficiency is determined in 20 bins and the migration matrix for $20 \times 20$ bins leading to 20 bins for the signal template. Since we use only five bins in our measurement, the number of bins is reduced after the calculation of the signal template with 20 bins by a factor of four. However, this procedure minimizes the effect of different 

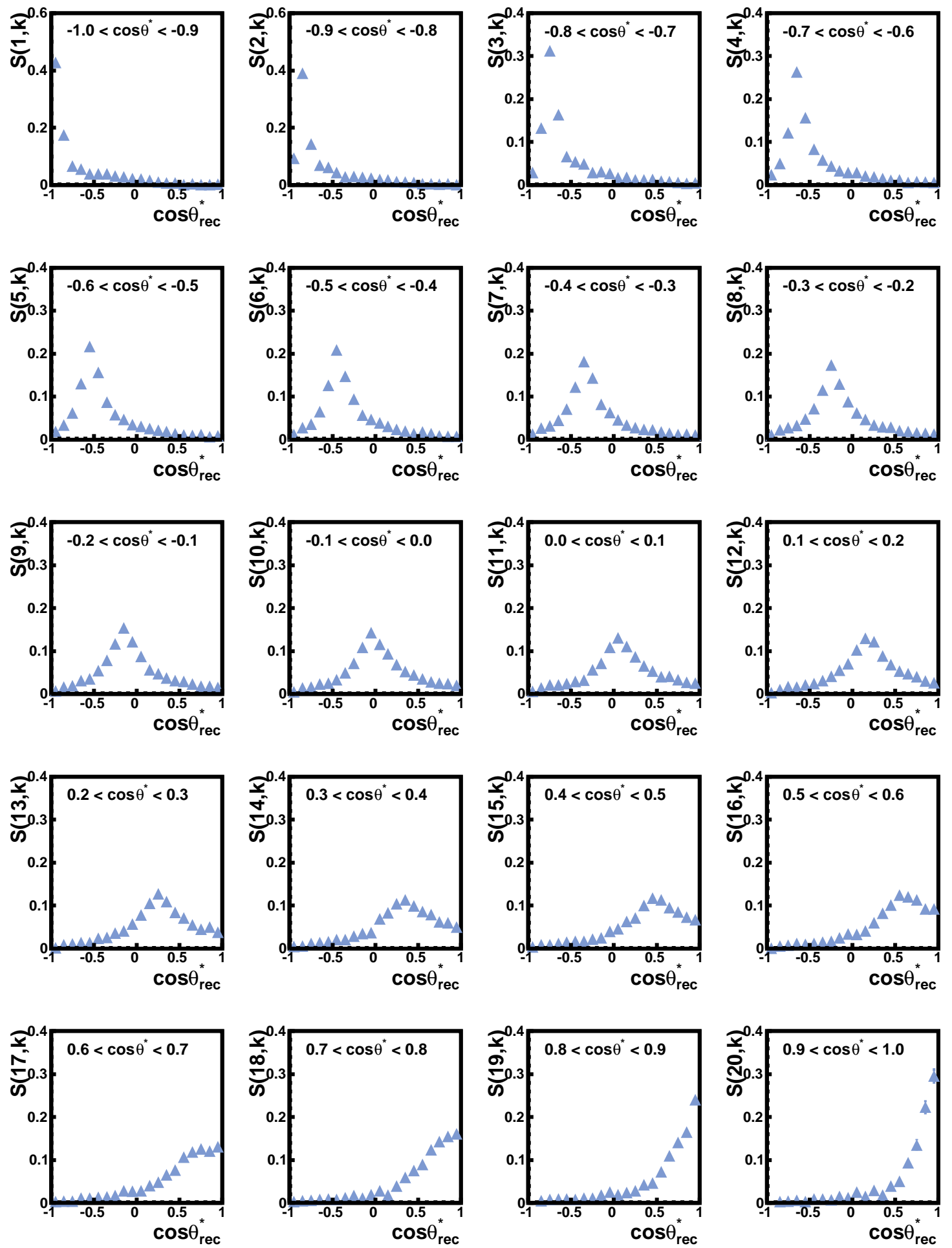

Figure 5.10: Migration matrix element $S(i, k)$ as a function of $\cos \theta_{\text {rec }}^{*}$ in 20 bins of $\cos \theta^{*}$ for

Standard Model polarized $W$ bosons as determined from a SM Monte Carlo sample generated with PyTHIA. 
efficiencies and migration matrix elements of differently polarized $W$ bosons due to the different $\cos \theta^{*}$ distribution.

The normalized signal templates computed from a SM PYTHIA sample as a function of $F_{0}\left(F_{+}=0\right)$ are shown in figure 5.11, while the templates as a function of $F_{+}\left(F_{0}=0.7\right)$ are presented in figure 5.12.

Figure 5.11 shows the influence of increasing $F_{0}$ on the expected number of signal events in each bin $k$ of the $\cos \theta^{*}$ distribution. Since $F_{+}$is fixed to zero, for small values of $F_{0}$ the first two bins contain the most events due to the large fraction of left handed $W$ bosons $F_{-}=1-F_{+}$whose $\cos \theta^{*}$ distribution peaks in this region as can be seen in figure 5.2. With increasing $F_{0}$ the contents in the first two bins decrease while the contents in the other three bins which are dominated by the distribution for longitudinally polarized $W$ bosons in absence of right handed $W$ bosons increase. The signal template with the steepest gradient is the template for the first bin, thus the expected number of events in this bin is very sensitive to $F_{0}$. For example, values of $F_{0}$ of 0.5 or 0.9 instead of the SM expectation 0.7 lead to an increase or decrease in the expected number of events of about $25 \%$.
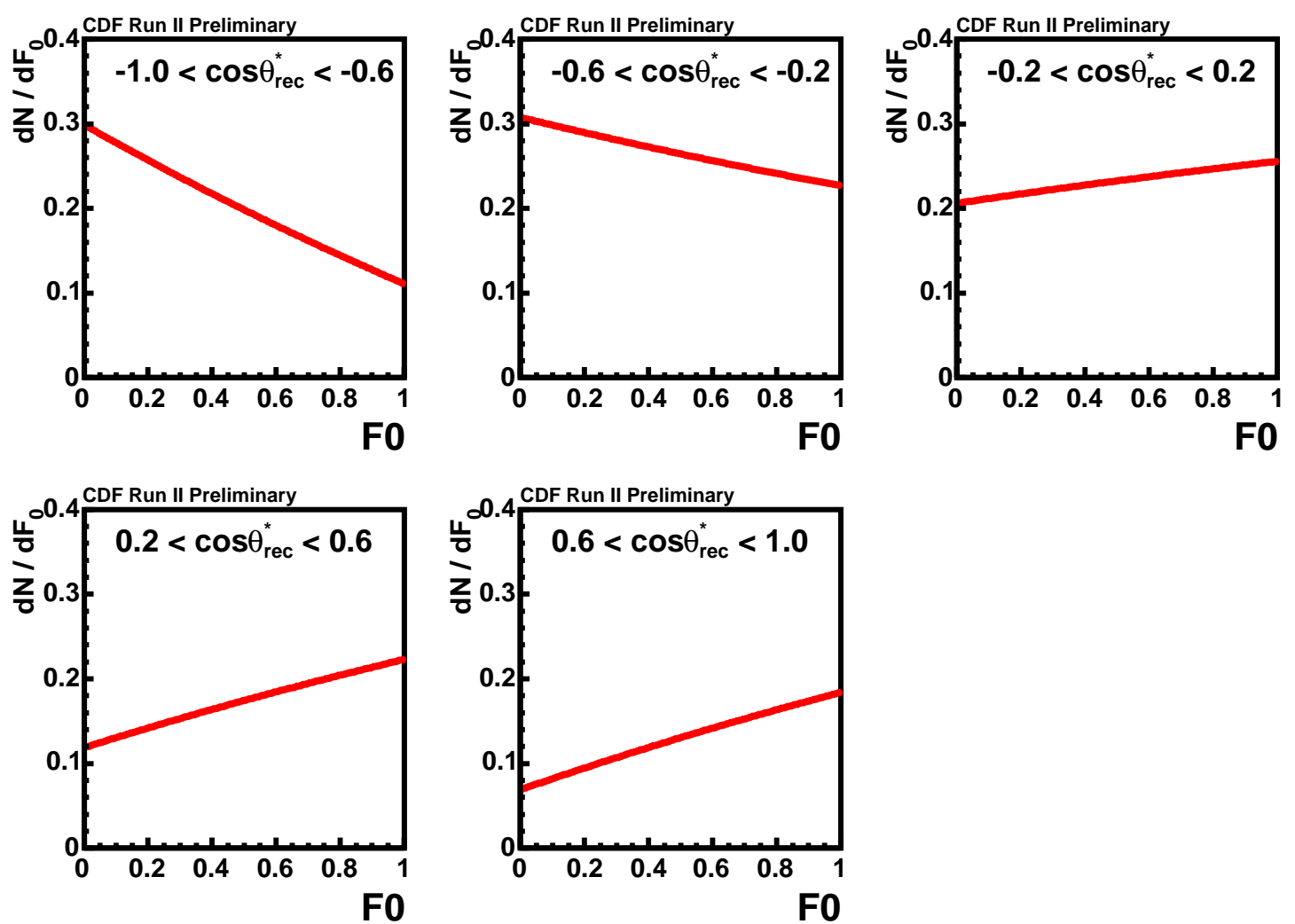

Figure 5.11: Dependence of the normalized number of events in the five $\cos \theta_{\text {rec }}^{*}$ bins on $F_{0}$. Here $F_{+}$is fixed to its SM value zero. The fit templates are calculated from the efficiency and the migration matrix obtained from a SM Monte Carlo sample generated with PYTHIA. 
To derive the fit templates for the measurement of $F_{+}$the fraction $F_{0}$ is fixed to its $\mathrm{SM}$ value 0.7 . With increasing $F_{+}$the number of expected events in bin four and five increases, since the $\cos \theta^{*}$ distribution for right handed $W$ bosons peaks in that region as can be seen in figure 5.2. At the same time $F_{-}=0.7-F_{+}$and therefore the expected number of events in the first two bins decreases. In the middle bin the distributions for left and right handed $W$ bosons are negligible compared to the distribution for longitudinally polarized $W$ bosons, and therefore the signal template for this bin is nearly independent on $F_{+}$.
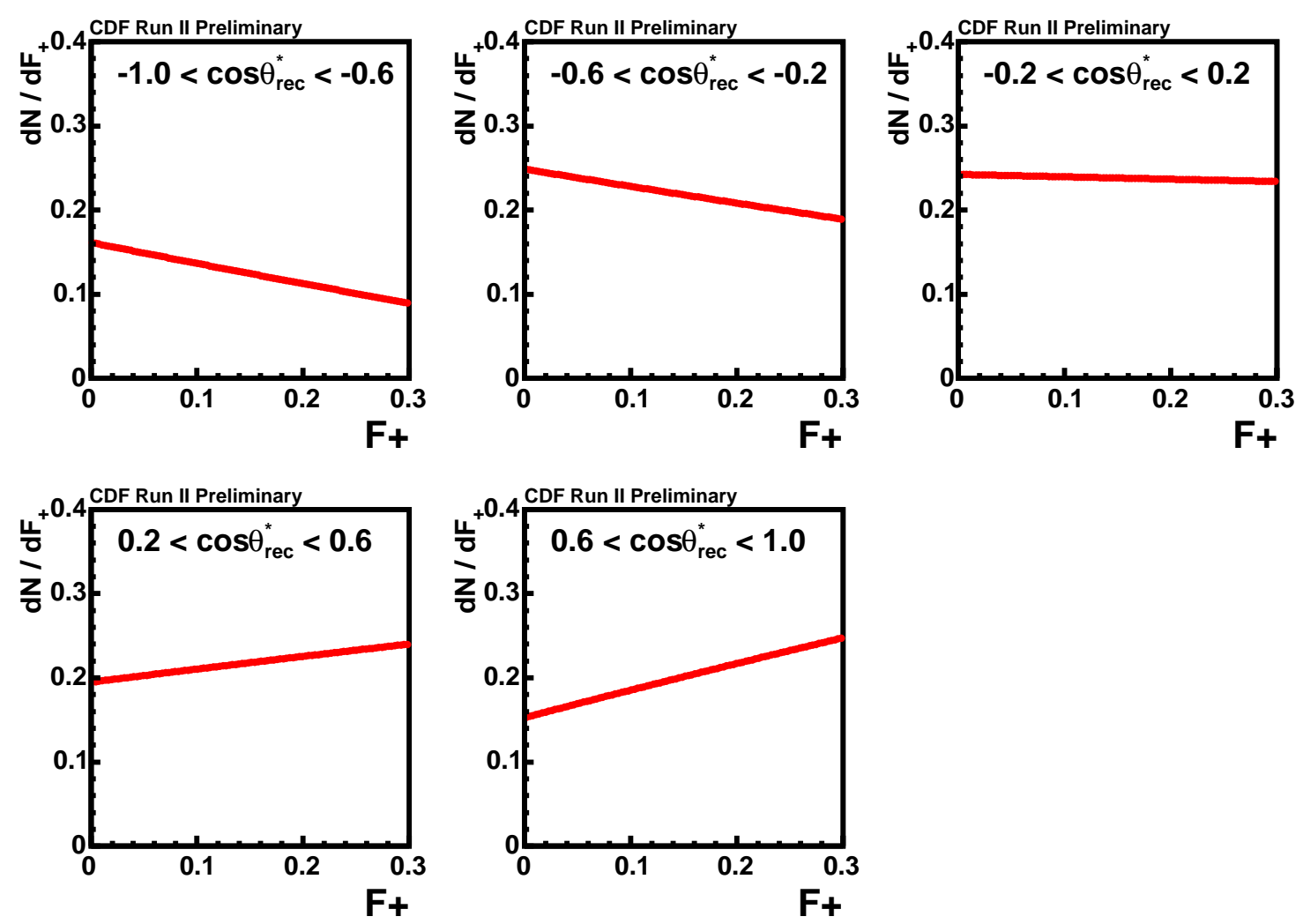

Figure 5.12: Dependence of the normalized number of events in the five $\cos \theta_{\text {rec }}^{*}$ bins on $F_{+}$. Here $F_{0}$ is fixed to its SM value 0.7. The fit templates are calculated from the efficiency and the migration matrix obtained from a SM Monte Carlo sample generated with PYTHIA. 


\subsection{Likelihood Fit}

Due to the small number of bins used for the observed $\cos \theta^{*}$ distribution the number of degrees of freedom is already small. In order to reduce this number not further and since the number of $t \bar{t}$ candidates in the data is still small, we do not extract simultaneously the fraction of longitudinally polarized $W$ bosons and right handed $W$ bosons. Instead we fix first the right handed helicity fraction $F_{+}$to zero and afterwards $F_{0}$ to 0.7 , as predicted both in the Standard Model. Thus, only one free parameter is used in both fits. This treatment can be seen as a test of two physical models with anomalous couplings which are discussed in 1.3. In the case where $F_{0}$ is fixed to the SM value 0.7 the possible $\mathrm{V}+\mathrm{A}$ component in the structure of the weak interaction at high energies is tested, while in the case of fixed $F_{+}$the anomalous magnetic coupling $f_{2}^{R}$ is tested under the assumption that no couplings to right handed $b$ quarks exist. To extract the single free parameter $\left(F_{0}\right.$ or $\left.F_{+}\right)$we use a binned maximum likelihood method [74]. The likelihood function $L(a)$ in which $a$ is the free parameter is calculated by :

$$
L(a)=\prod_{k=1}^{N_{\text {bins }}} \frac{\mu_{k}^{\text {obs }}(a)^{n_{k}} \cdot e^{-\mu_{k}^{\text {obs }}(a)}}{n_{k} !} .
$$

Here, $\mu_{k}^{\text {obs }}$ denotes the number of events expected to be observed in bin $k$ of the reconstructed $\cos \theta_{\text {rec }}^{*}$ distribution and $n_{k}$ the measured number of events in the same bin. In order to get $F_{0}$ or $F_{+}$respectively, we minimize the negative logarithm of the likelihood function $L(a)$ by varying the free parameter $F_{0}$ or $F_{+}$.

The expected number of events in bin $k \mu_{k}^{\text {obs }}$ is the sum of the expected number of $t \bar{t}$ signal events $\mu_{k}^{\text {sig,obs }}$ and the expected number of background events $\mu_{k}^{\mathrm{BG} \text {,obs }}$ :

$$
\mu_{k}^{\mathrm{obs}}=\mu_{k}^{\mathrm{sig}, \mathrm{obs}}+\mu_{k}^{\mathrm{BG}, \mathrm{obs}} .
$$

The expected number of signal events in bin $k$ is:

$$
\mu_{k}^{\text {sig,obs }}\left(F_{0}, F_{+}\right)=N_{\text {data }} \cdot f_{\text {sig }} \cdot \hat{\mu}_{k}^{\text {sig,obs }}\left(F_{0}, F_{+}\right),
$$

where $\hat{\mu}_{k}^{\mathrm{sig}, \mathrm{obs}}$ is the signal template for the $k^{\text {th }}$ bin of $\cos \theta_{\mathrm{rec}}^{*}$ as derived in the previous section (see equations 5.17 and 5.21). $N_{\text {data }}$ is the number of observed events in the selected data sample and $f_{\text {sig }}$ the fraction of signal events, which is derived from the total number of reconstructed events and the background estimate, leading to $f_{\text {sig }}=87 \%$. Thus, the product $N_{\text {data }} \cdot f_{\text {sig }}$ gives the expected number of signal events in the observed data.

In the case, where $F_{+}$is set to zero, the fraction $F_{-}$of left handed $W$ bosons is $F_{-}=1-F_{0}$. In the second case, in which $F_{0}$ is fixed to the SM value of $0.7, F_{-}$is given by $F_{-}=0.3-F_{+}$.

For both fits the theoretically expected number of background events in bin $k$ of the reconstructed $\cos \theta_{\text {rec }}^{*}$ distribution is:

$$
\mu_{k}^{\mathrm{BG}, \mathrm{obs}}=N_{\text {data }} \cdot\left(1-f_{\mathrm{sig}}\right) \cdot f_{k}^{\mathrm{BG}} .
$$


Here, $f_{k}^{\mathrm{BG}}$ is the fraction of background events contained in bin $k$ of the normalized $\cos \theta^{*}$ distribution for background and $N_{\text {data }} \cdot\left(1-f_{\text {sig }}\right)$ is the number of estimated background events.

\subsection{Results}

Performing the fit with fixed $F_{+}=0$ the fraction $F_{0}$ of the longitudinally polarized $W$ bosons is determined to be $F_{0}=0.59 \pm 0.12$ (stat.), which is consistent with the Standard Model prediction of 0.7. For the second fit, where $F_{0}$ is fixed to its SM value of 0.7 , a fraction of right handed $W$ bosons of $F_{+}=-0.03 \pm 0.06$ (stat.) is obtained which is again in good agreement with the SM expectation. In figure 5.13 (a) and (b) the negative logarithm of the likelihood function as a function of $F_{0}$ and $F_{+}$, respectively, is shown, in which the minimum represents the result of the fit.

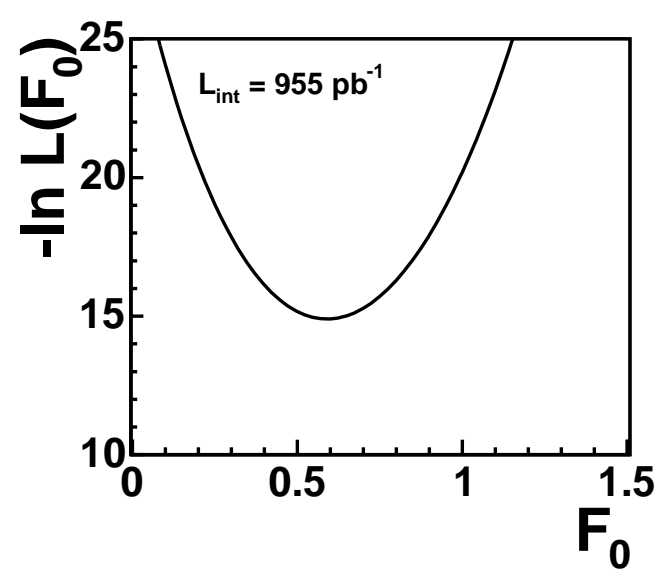

(a)

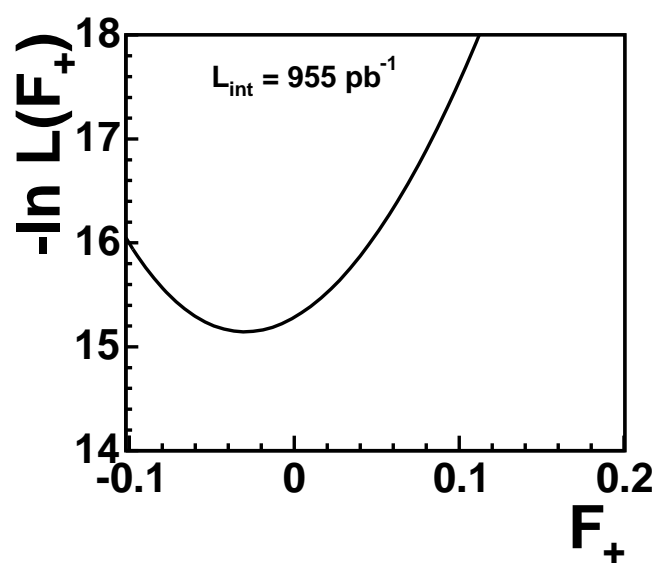

(b)

Figure 5.13: Negative log likelihood function as a function of $F_{0}$ and $F_{+}$, respectively. (a) For the extraction of $F_{0}$, the right handed fraction is set to zero, as expected in the Standard Model. (b) For the extraction of $F_{+}, F_{0}$ is set to 0.7 , as expected in the Standard Model.

Since no evidence for a nonzero fraction of right handed $W$ bosons is found, an upper limit $F_{+}^{\max }$ for $F_{+}$at the $95 \%$ confidence level (C.L.) is calculated by integrating the likelihood function $L\left(F_{+}\right)$. Since a Bayesian approach is pursued, we integrate only in the physical region $0 \leq F_{+} \leq 0.3$ applying a prior distribution which is 1 in the interval $[0,0.3]$ and 0 elsewhere. 


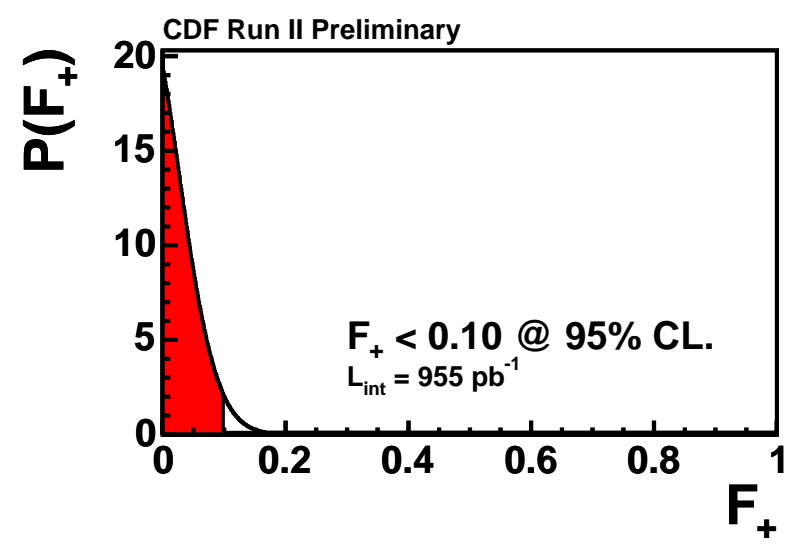

Figure 5.14: Posterior probability density $P\left(F_{+}\right)$as a function of the right handed fraction $F_{+}$. The region excluded at the $95 \%$ C.L. is indicated in white.

$$
\text { C.L. }\left(F_{+} \leq F_{+}^{\max }\right)=\frac{\int_{0}^{F_{+}^{\max }} L\left(F_{+}\right) d F_{+}}{\int_{0}^{0.3} L\left(F_{+}\right) d F_{+}}:=0.95
$$

We obtain an upper limit on the fraction of right handed $W$ bosons of $F_{+} \leq 0.10$ at the $95 \%$ C.L. The probability density $P\left(F_{+}\right)=L\left(F_{+}\right) / \int_{0}^{0.3} L\left(F_{+}\right) d F_{+}$is presented in figure 5.14 with the region excluded at the $95 \%$ C.L. indicated in white.

\subsection{Unfolded $\cos \theta^{*}$ Distribution}

In order to allow a direct comparison of the $\cos \theta_{\text {rec }}^{*}$ distribution obtained from the selected data sample with the calculated distributions for the different $W$ boson helicity modes presented in figure 5.2, the background estimate is subtracted from the data. The shape of the $\cos \theta_{\text {rec }}^{*}$ distribution is then corrected for acceptance effects as well as for resolution effects applying the transfer function $\tau\left(F_{0}, F_{+}\right)$.

Since the transfer function explicitly depends on $F_{0}$ and $F_{+}$for the correction of the data distribution the specific transfer function for the $F_{0}$ value obtained by the likelihood fit has to be used, while the other parameter $F_{+}$is set to its SM value, thus $\tau\left(F_{0}=F_{0}^{\mathrm{fit}}, F_{+}=0\right)$. In a similar way the data can be corrected by using the result from the likelihood fit for $F_{+}$and setting $F_{0}$ to its SM value, thus $\tau\left(F_{0}=0.7, F_{+}=F_{+}^{\mathrm{fit}}\right)$.

The value of the transfer function in the $i^{\text {th }}$ bin of $\cos \theta^{*}$ is calculated from the normalized number of events $\hat{\mu}_{i}^{\text {sig }}$ before applying any selection cuts in the $i^{\text {ith }}$ bin of $\cos \theta^{*}$ and from the normalized number of events $\hat{\mu}_{k}^{\text {sig,obs }}$ after applying the selection cuts and performing the reconstruction in the $k^{\text {th }}$ bin of $\cos \theta_{\text {rec }}^{*}$ : 


$$
\tau_{i}\left(F_{0}, F_{+}\right)=\frac{\hat{\mu}_{i}^{\mathrm{sig}}\left(F_{0}, F_{+}\right)}{\hat{\mu}_{k=i}^{\mathrm{sig}, \mathrm{obs}}\left(F_{0}, F_{+}\right)} .
$$

Multiplying the background subtracted number of events in bin $k(k=i)$ of $\cos \theta_{\text {rec }}^{*}$ with $\tau_{i}\left(F_{0}^{\text {fit }}, F_{+}=0\right)$ or $\tau_{i}\left(F_{0}=0.7, F_{+}^{\text {fit }}\right)$ respectively and normalizing subsequently the corrected $\cos \theta^{*}$ data distribution to the theoretically calculated $t \bar{t}$ pair production cross section of $\sigma_{t \bar{t}}=6.7 \pm 0.9 \mathrm{pb}[18,19]$, leads to the desired distribution which is directly comparable with the theory distributions.

In figure 5.15 the corrected $\cos \theta^{*}$ distribution of the data normalized to the $t \bar{t}$ pair production cross section is presented. The uncertainty in the data is due to the uncertainty of the transfer function. Figure 5.15 (a) and (b) also provide in red the distributions corresponding to the fitted value for $F_{0}$ and $F_{+}$respectively. In order to compare the results with the SM prediction, figure 5.15 (a) also provides the distribution for the SM value of $F_{0}$ in blue dashed. In (b) two distributions calculated from two different nonzero values of $F_{+}$, namely $F_{+}=0.1$ and $F_{+}=$ 0.2 , are represented by the dashed lines. Here it becomes apparent that, under the assumption of $F_{0}=0.7$, the distribution in the data is not compatible with distributions corresponding to values for $F_{+}$which deviate significantly from zero.

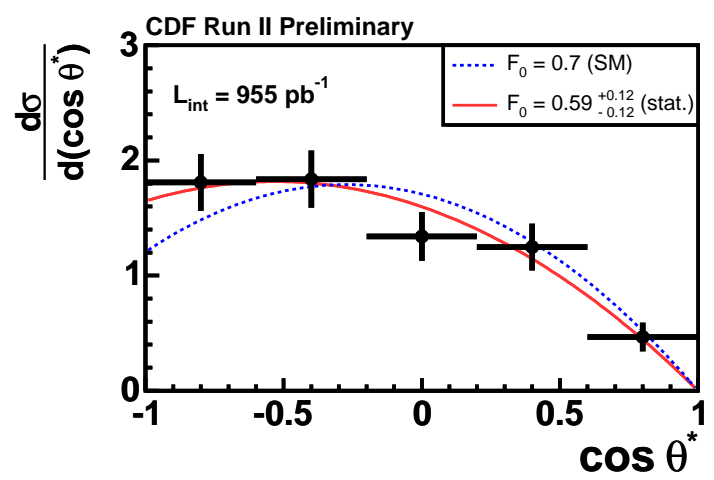

(a)

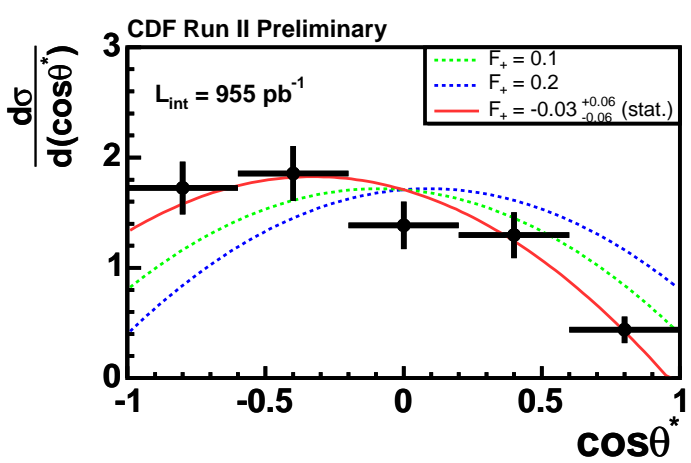

(b)

Figure 5.15: Unfolded $\cos \theta^{*}$ distributions in data normalized to the theoretically calculated $t \bar{t}$ pair production cross section of $\sigma_{t \bar{t}}=6.7 \pm 0.9 \mathrm{pb}[18,19]$. (a) shows the unfolded $\cos \theta^{*}$ distribution derived by applying the transfer function obtained from the fitted value of $F_{0}\left(F_{+}=0\right)$. (b) displays the distribution unfolded with the transfer function obtained from the fitted value of $F_{+}$ $\left(F_{0}=0.7\right)$. In both plots the black points represent the data, while the red smooth curves represent the theoretical distributions corresponding to the fitted values for $F_{0}$ and $F_{+}$respectively. The dashed lines represent distributions for different values of $F_{0}$ and $F_{+}$, respectively. 


\section{Chapter 6}

\section{Verification of our Method and Systematic Uncertainties}

After having described our method for the measurement of the $W$ helicity fractions in the previous chapter, we verify this method in this chapter and determine the sensitivity. Furthermore the different sources of systematic uncertainties that have to be considered are discussed and quantified. Since for both purposes pseudo experiments are performed, we start with a description of this technique.

\subsection{Pseudo Experiments}

Performing pseudo experiments means to repeat the measurement for an ensemble of simulated data samples drawn from a MC sample using the same statistics as in the real data sample. This procedure offers the opportunity to obtain a whole series of simulated measurements and to see how the results from the real measurement are compared to the average. Since for the sake of realistic simulation the statistics in a simulated sample are limited, using a whole ensemble of simulated data samples ensures that the results are independent from statistical fluctuations. In case of systematic studies, for example, only the effects due to different systematic settings are measured, since statistical fluctuations cancel out.

For each pseudo experiment the number of background events in the simulated data sample is thrown according to a Poisson distribution with a mean of 6.8 for non-W events, a mean of 12.25 for the mistag background and with a mean of 12.27 for the background from diboson, $W$ +heavy flavor, and single-top production. The used values correspond to the background estimation presented in section 3.4. The number of signal events in the simulated data sample is thrown according to a Poisson distribution with a mean of $232-31.32=200.68$ which is the difference between the number of $t \bar{t}$ candidates found in the real data sample and the estimated number of background events. Only such combinations are taken, in which the total number of thrown events is 232 . Then we create the reconstructed $\cos \theta_{\text {rec }}^{*}$ distribution for each pseudo experiment. Since only this distribution and no other kinematic quantities of the entire event are of interest for our studies, we do not select randomly 
the thrown number of signal and background events from the $t \bar{t}$ signal MC sample and the background samples and reconstruct the $\cos \theta^{*}$ distribution from the selected events, but throw the number of signal events according to the reconstructed $\cos \theta_{\text {rec }}^{*}$ distribution obtained from the $t \bar{t}$ signal MC sample and the number of background events according to the background templates.

The same maximum likelihood fit as used for the real data is then applied to the reconstructed $\cos \theta_{\text {rec }}^{*}$ distribution obtained in this way in order to get the helicity fractions. Each pseudo experiment delivers one extracted value for $F_{0}$ and one for $F_{+}$. Also the statistical uncertainty $\sigma$ for each pseudo experiment can be determined. Since a whole ensemble of experiments is simulated, this leads to distributions for $F_{0}$ and $F_{+}$as well as for $\sigma$.

\subsection{Verification of our Method}

In order to verify our analysis method, different kinds of checks are performed, using different ensembles of pseudo experiments obtained from $\mathrm{MC}$ samples generated with different settings. First a consistency check using a standard $t \bar{t}$ signal MC sample generated with PYTHIA is performed. In this procedure we determine also the sensitivity of our method. In a second step pseudo experiments from GGWIG samples are used to extend the test range to possible non-SM values of the helicity fractions.

\subsubsection{Consistency Check}

For the consistency check the events for the pseudo experiments are drawn from the same PYTHIA MC sample that was used to determine the efficiency and migration matrix for the calculation of the fit templates, described in the previous chapter. Since we have 70400 events in this MC sample after the event selection and 232 events in the selected data sample and to avoid oversampling, we perform 303 pseudo experiments. As mentioned above we get one value for $F_{0}$ and one value for $F_{+}$from each pseudo experiment.

The quality of our method to measure $F_{0}$ and $F_{+}$is then checked in detail with the resulting pull distributions. The pull is defined as $\left(F_{0 /+}^{\mathrm{fit}}-F_{0 /+}^{\mathrm{input}}\right) / \sigma_{F_{0 /+}^{\mathrm{fit}}}$, thus the difference of the extracted $\left(F_{0 /+}^{\mathrm{fit}}\right)$ and input $\left(F_{0 /+}^{\text {input }}\right)$ value for each pseudo experiment divided by the statistical uncertainty of the extracted $F_{0 /+}$. For a consistent method the mean of the obtained pull distribution is expected to be zero and the width $\sigma$ of the pull distribution should be one, meaning that the error estimation of the method is correct. In figure 6.1 the corresponding pull distributions for the measurement of $F_{0}$ and $F_{+}$are presented. Both pull widths, the root mean squares of the pull distributions, are consistent with one as required and the pull means are also consistent with zero within the statistical errors. 


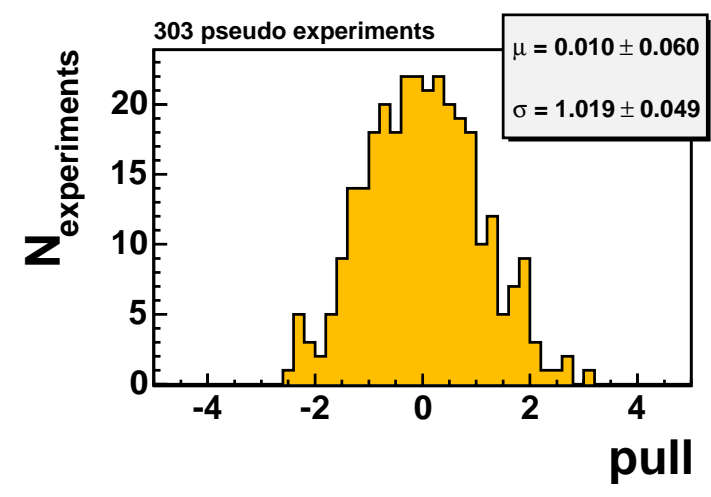

(a)

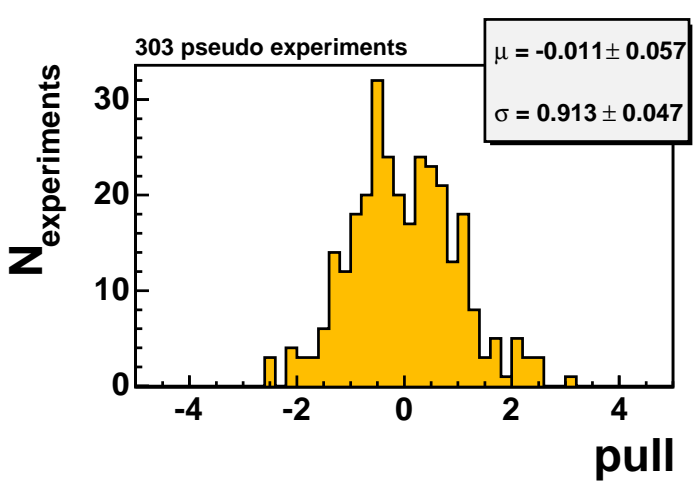

(b)

Figure 6.1: Pull distributions $\left(\left(F_{0 /+}^{\mathrm{fit}}-F_{0 /+}^{\mathrm{input}}\right) / \sigma_{F_{0 /+}^{\mathrm{fit}}}\right)$ for the measurement of (a) $F_{0}$ and (b) $F_{+}$ for 303 pseudo experiments performed using the same statistics of data as in the real experiment and using a $t \bar{t}$ signal MC sample generated with PyтнIA. $\mu$ denotes the mean and $\sigma$ the root mean square of the pull distribution.

\subsubsection{Sensitivity}

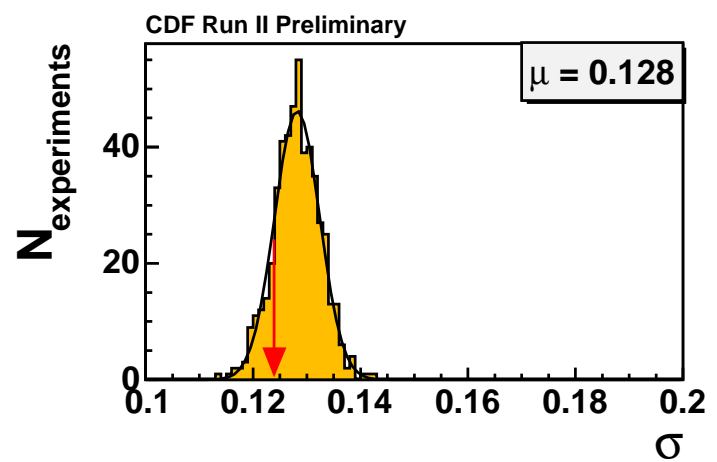

(a)

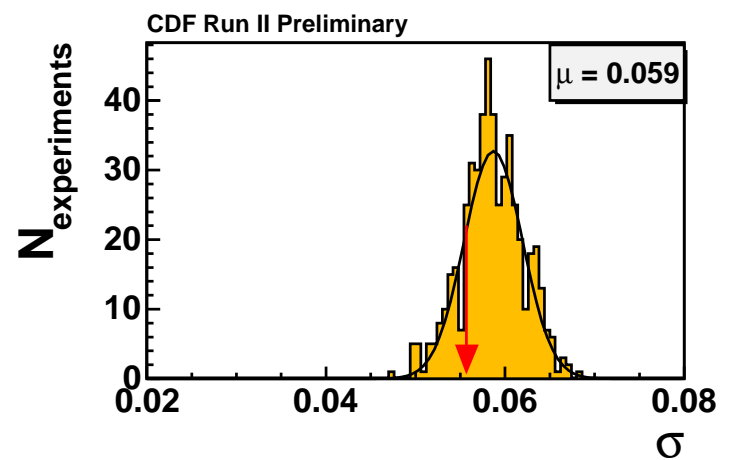

(b)

Figure 6.2: Expected sensitivity for the measurement of (a) $F_{0}$ and (b) $F_{+}$obtained from pseudo experiments. The expected sensitivity is given by the mean $\mu$ of the Gaussian fitted to the distribution. The statistical uncertainty of the real measurement in data is indicated by the red arrow.

The expected sensitivity of a certain method is determined using pseudo experiments. Each pseudo experiment delivers one statistical uncertainty $\sigma$ for the measurement of either $F_{0}$ or $F_{+}$which leads to a distribution of $\sigma$. The mean value of a Gaussian fitted to the obtained distribution then represents the expected sensitivity 


\section{Chapter 6. Verification of our Method and Systematic Uncertainties}

of the utilized method. In case of our method for the measurement of $F_{0}$ and $F_{+}$ the expected sensitivities are 0.128 and 0.059 respectively.

Now we can compare the statistical uncertainty of our single real measurement with the expected sensitivity, in other words, we can compare our statistical uncertainty with the average of many simulated experiments. Figure 6.2 shows the $\sigma$ distributions for the measurements of $F_{0}$ and $F_{+}$together with the actual statistical uncertainties of our measurements, indicated by the red arrows. The statistical uncertainties of our single real measurement are 0.124 for the measurement of $F_{0}$ and 0.056 for the measurement of $F_{+}$and are in both cases slightly smaller than the average.

\subsubsection{Further Checks}

In order to simulate non Standard Model values of $F_{0}$ or $F_{+}$, the independent GGWIG Monte Carlo samples are used, in which one $W$ boson is fixed to be left handed, right handed or longitudinally polarized. The reconstructed $\cos \theta_{\text {rec }}^{*}$ distribution for arbitrary values of $F_{0}$ and $F_{+}$is obtained by mixing these three samples according to the desired $F_{0}$ and $F_{+}$.

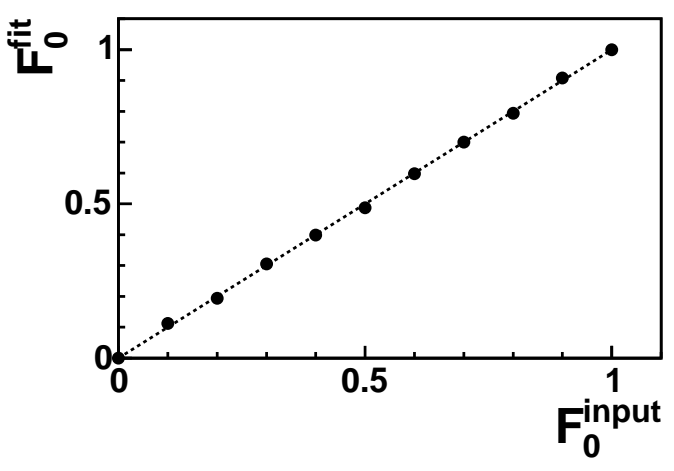

(a)

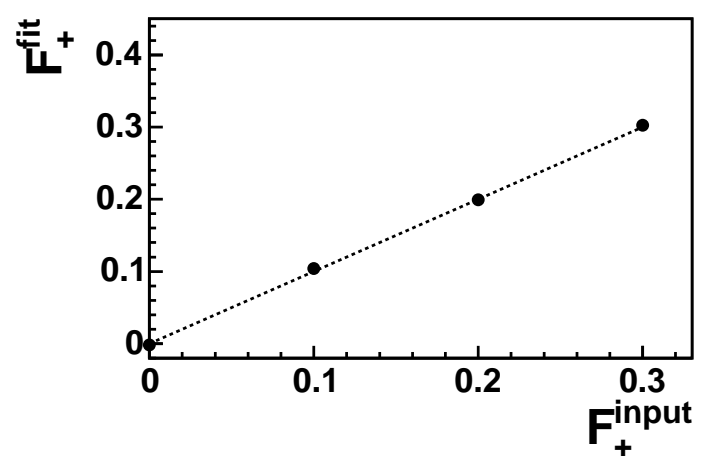

(b)

Figure 6.3: Extracted $W$ helicity fractions (a) $F_{0}^{\text {fit }}$ and (b) $F_{+}^{\text {fit }}$, i.e. the mean values of the corresponding $F_{0 /+}$ distributions obtained from pseudo experiments, versus the $W$ helicity fractions (a) $F_{0}^{\text {input }}$ and (b) $F_{+}^{\text {input }}$ chosen to be present in the test MC sample. The pseudo experiments are drawn from a combination of the different GGWIG samples, according to the values of $F_{0 /+}^{\text {input }}$, using the same statistics of data as in the real measurement.

For several $F_{0}\left(F_{+}\right)$input values pseudo experiments are performed and the extracted $F_{0}^{\mathrm{fit}}\left(F_{+}^{\mathrm{fit}}\right)$ value is compared to the input value. The $\cos \theta_{\mathrm{rec}}^{*}$ distribution for the signal events is thrown according to the mixed GGWIG sample. The fit is performed using the signal templates calculated from the efficiencies and migration 
matrix elements obtained from the SM $W$ bosons of the GGWIG samples and not from the default PYTHIA sample. This is done to avoid systematic effects arising from differences between different Monte Carlo generators. The result is presented in figure 6.3. Good agreement between the input values and the extracted values can be observed.

In figure 6.4 the means and widths, i.e. the root mean squares, of the pull distributions as a function of $F_{0}$ and $F_{+}$are presented. For $F_{0}$ as well as for $F_{+}$the pull means are consistent with zero and the pull widths are consistent with the expected value of one.

(a)

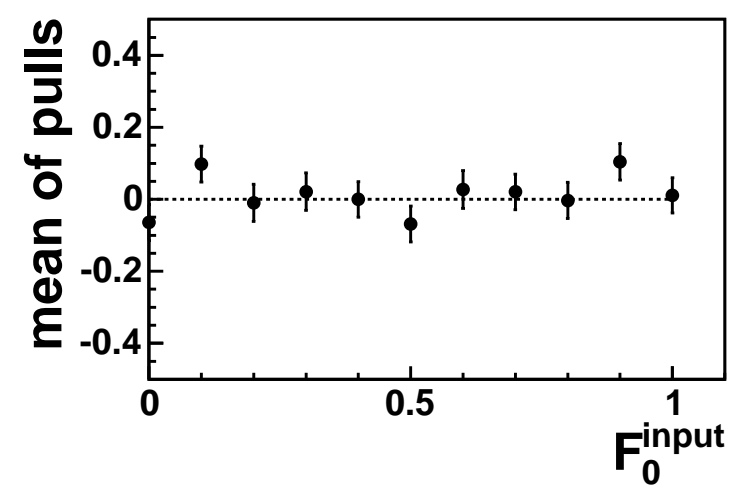

(c)

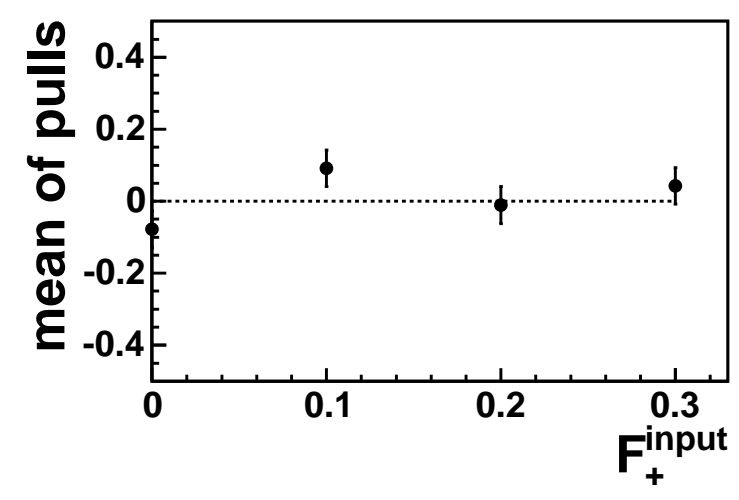

(b)

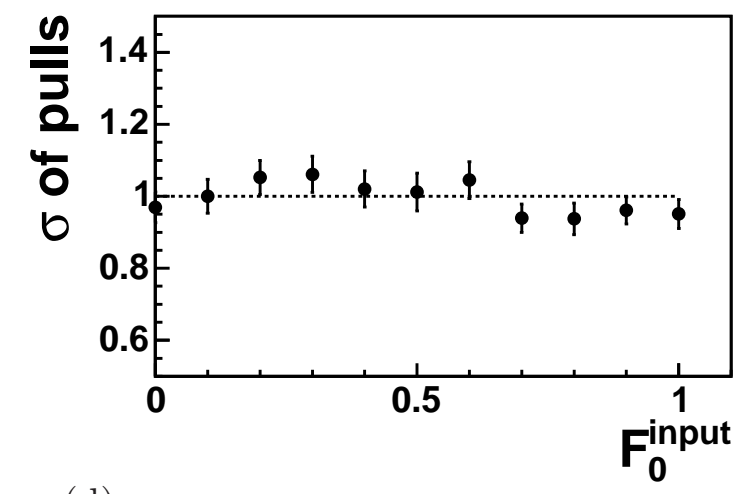

(d)

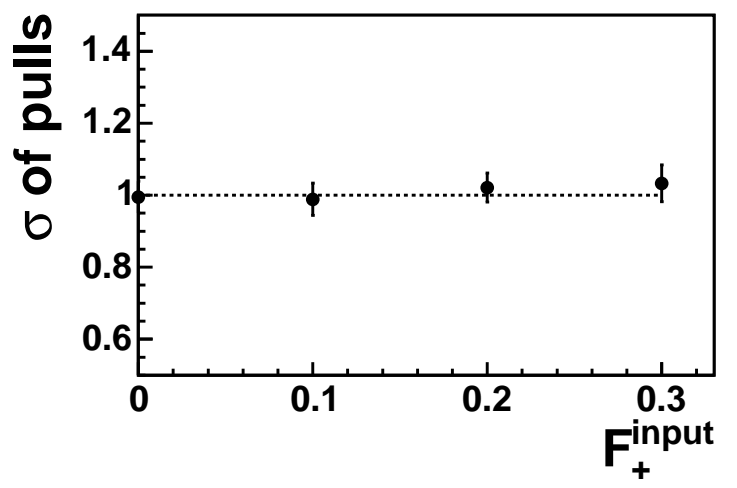

Figure 6.4: (a) $+(\mathrm{c})$ Means and $(\mathrm{b})+(\mathrm{d})$ widths (root mean squares) of the pull distributions $\left(\left(F_{0 /+}^{\mathrm{fit}}-F_{0 /+}^{\text {input }}\right) / \sigma_{F_{0 /+}^{\mathrm{fit}}}\right)$ as $(\mathrm{a})+(\mathrm{b})$ a function of $F_{0}^{\text {input }}$ as well as $(\mathrm{c})+(\mathrm{d})$ a function of $F_{+}^{\text {input }}$.

However, since the statistics in the GGWIG samples are very limited, we are forced to calculate the fit templates from an efficiency distribution in five instead of 20 bins and a $5 \times 5$ instead of $20 \times 20$ migration matrix and therefore the obtained check results are only a lower limit for the quality of our method. 


\subsection{Systematic Uncertainties}

The systematic uncertainties caused by theoretical modeling, the experimental setup, and the analysis method are studied using ensembles of simulated experiments. For these studies we use 600 pseudo experiments corresponding to an arguable oversampling factor of two to make the results more stable. In the following the determination of the systematic uncertainties listed in table 6.1 is described in more detail.

\section{Theory based Uncertainties}

In case of the theory based uncertainties, systematic uncertainties caused by the imperfect knowledge of the parton density functions (PDF), the uncertainties of the initial and final state radiation (ISR+FSR) modelling, and the uncertainty of the calculation of the $t \bar{t}$ kinematics are investigated. In order to study such effects, Monte Carlo simulations with changed PDF, ISR+FSR treatment and a different Monte Carlo generator, namely HERWIG, are used. The number of signal events is thrown according to the reconstructed $\cos \theta_{\text {rec }}^{*}$ distribution obtained from a MC sample generated with modified settings, while the fit procedure remains unchanged. The fit templates are calculated using the efficiency and migration matrix obtained from the same SM PyTHIA sample as used in the real measurement and in order to extract either $F_{0}$ or $F_{+}$the other parameter is set to its SM value.

\section{- Monte Carlo (MC) Generator}

We account for possible bias from MC modeling of $t \bar{t}$ events by comparing HERWIG and PYTHIA event generators. Therefore the simulated events are drawn either from a MC sample generated with PYTHIA or from a MC sample generated with HERWIG, while as mentioned above, for the calculation of the fit templates in both cases the MC sample generated with PyTHIA is used.

\section{- Parton Distribution Function (PDF)}

The contribution of the parton distribution function (PDF) uncertainty is determined using $t \bar{t}$ signal MC samples generated with two different PDFs, namely MRST72 and MRST75 [75], while CTEQ5L [76] is used for the nominal value.

\section{- Initial and Final State Radiation (ISR, FSR)}

To estimate the influence of initial state and final state radiation we use templates from Pyтhia Monte Carlo simulations in which the parameters for gluon radiation are varied to produce either less or more initial or final-state radiation compared to the standard setup. 
The systematic uncertainties arising from theoretical uncertainties are symmetrized and summarized in the upper part of table 6.1 .

\section{Uncertainties based on Experimental Setup and Analysis Method}

Concerning the systematic uncertainties caused by the experimental setup and the analysis method we study the impact of the variation of the jet energy corrections according to their uncertainties stated in [66] and the impact of the normalization and shape uncertainty of the background. These effects are again studied using pseudo experiments. The determined uncertainties of these sources are summarized in the lower part of table 6.1 .

\section{- Jet Energy Scale}

The uncertainty due to the jet energy scale is quantified by varying that correction within one standard deviation in both the negative and positive direction. The selected events and their reconstructed kinematics therefore slightly change and thus also the reconstructed $\cos \theta_{\text {rec }}^{*}$ distribution is altered.

\section{- $b$ Probability}

To study the impact of the $b$-likeness information used in the calculation of our quantity $\Psi$, the $P_{\mathrm{b} \text {-light }}$ term in the formula for $\Psi$ (equation 4.2 ) is omitted. The results for pseudo experiments in which the hypothesis selection is based on this shortened calculation of $\Psi$ are then compared to results obtained from pseudo experiments in which the default hypothesis selection is performed.

\section{- Background Rate}

To estimate the contribution of the background rate uncertainty we simultaneously add or subtract, respectively, the values of one standard deviation of the estimated rates for the non-W events $(6.8 \pm 1.8)$, the mistag background $(12.25 \pm 1.83)$, and the background events due to $W$ +heavy flavor, diboson and single-top production $(12.27 \pm 2.48)$. For the pseudo experiments we throw again the same number of events as in the data, but the number of background events is now taken Poisson distributed with the increased or decreased means respectively. The extraction of $F_{0}$ and $F_{+}$is then performed as in the default case, meaning that here the default background estimate is used. 


\section{- Background Shape Modeling}

For the analysis three different background samples are used, the $W+4$ light quark Monte Carlo sample, the $W+2 b$ quarks+2 light quarks Monte Carlo sample, and the non-W multi-jet sample. The uncertainty due to the background shape uncertainty is estimated by using each shape of the three background distributions alone instead of using a composition of these shapes. The reconstructed $\cos \theta_{\text {rec }}^{*}$ distribution for the background events is here thrown according to the modified distribution obtained by using only one background sample. The extraction of $F_{0}$ and $F_{+}$is then performed as in the default case, meaning that here the default shape of the background estimate is used. The uncertainty obtained from the case with the largest deviation in $F_{0}$ and $F_{+}$respectively compared to the values obtained from the default background composition is then stated.

\section{- Lepton Energy}

The impact of the uncertainty on the measurement of the energy of the charged lepton is estimated by varying this energy by one percent in both directions. However, the influence of this uncertainty on the result of our measurement is only tiny.

\section{- High Instantaneous Luminosity}

The number of minimum bias events per interaction increases with increasing instantaneous luminosity. The MC samples used in our analysis do not consider these minimum bias events, which does not matter for the 0d data samples, but could have an influence on our measurement, since in the most recent data samples higher instantaneous luminosities are achieved. We study the possible effect of minimum bias events on our measurement using three different MC samples with no, one, and two minimum bias events. Though no visible dependence on the number of minimum bias events is found, we estimate the contribution to the systematic uncertainty by taking the largest difference between the means of the pull distributions obtained from one of the $\mathrm{MC}$ samples containing minimum bias events and the default MC sample. However, the determined contribution to the total uncertainty is only tiny.

Since the fraction of longitudinally polarized $W$ bosons depends explicitly on the top quark mass, we do not include this dependence into the systematic uncertainties, but present our measurement assuming a certain top mass, namely $175 \mathrm{GeV} / c^{2}$.

By adding all single uncertainties in quadrature we obtain a total systematic uncertainty of +0.068 and -0.062 for the measurement of the fraction of longitudinally 
polarized $W$ bosons and for the measurement of the right handed fraction a systematic uncertainty of +0.039 and -0.033 is obtained. As can be seen in table 6.1 the systematic uncertainties are dominated by the jet energy-scale uncertainty which is about $90 \%$ of the total uncertainty.

\begin{tabular}{|l|cc|cc|}
\hline & \multicolumn{4}{|c|}{ Uncertainties } \\
Source & $-\Delta F_{0}$ & $+\Delta F_{0}$ & $-\Delta F_{+}$ & $+\Delta F_{+}$ \\
\hline \hline Monte Carlo Generator & 0.006 & 0.006 & 0.001 & 0.001 \\
Parton Density Functions & 0.007 & 0.007 & 0.002 & 0.002 \\
Initial State Radiation & 0.000 & 0.000 & 0.002 & 0.002 \\
Final State Radiation & 0.013 & 0.013 & 0.008 & 0.008 \\
\hline Jet Energy Scale & 0.057 & 0.063 & 0.030 & 0.036 \\
- likeness & 0.014 & 0.014 & 0.009 & 0.009 \\
Normalization of Background & 0.006 & 0.006 & 0.002 & 0.002 \\
Background Shape & 0.010 & 0.012 & 0.007 & 0.009 \\
Lepton Energy & 0.002 & 0.002 & 0.001 & 0.001 \\
Minimum Bias Events & 0.002 & 0.002 & 0.002 & 0.002 \\
\hline \hline Total & 0.062 & 0.068 & 0.033 & 0.039 \\
\hline
\end{tabular}

Table 6.1: Summary of systematic uncertainties; the total error is calculated by adding all single uncertainties in quadrature. 
90 Chapter 6. Verification of our Method and Systematic Uncertainties 


\section{Chapter 7}

\section{Conclusion and Outlook}

In this thesis, I have presented a method for the measurement of the fractions $F_{0}$ and $F_{+}$of longitudinally polarized and right handed $W$ bosons, respectively, in top quark decays using a selected sample with an integrated luminosity of approximately $955 \mathrm{pb}^{-1}$ collected with the CDF II detector. The first step was to select $t \bar{t}$ candidate events with a lepton+jets signature by requiring one charged lepton, at least four jets, and missing transverse energy in the event. For the measurement of the helicity fractions we used the cosine of the decay angle $\theta^{*}$ as sensitive observable. Since the measurement of $\cos \theta^{*}$ is based on fully reconstructed top quarks as well as on all decay products, it is important to reconstruct the entire event. Therefore, we determined the kinematic quantities of the $t \bar{t}$ pairs from the measured four-momenta of the decay particles. Since in this full reconstruction several ambiguities occur, for each event the interpretation that matched best the $t \bar{t}$ assumption had to be chosen. For the chosen reconstruction hypotheses we then calculated the values of $\cos \theta^{*}$ for each event and thus obtained the $\cos \theta^{*}$ distribution for the selected sample. Considering efficiency and migration effects we calculated fit templates from the theoretical $\cos \theta^{*}$ distributions for the three different helicity modes. For the extraction of $F_{0}$ and $F_{+}$a binned likelihood method was utilized.

Taking systematic uncertainties into account, assuming a top quark mass of $m_{t}=175 \mathrm{GeV} / c^{2}$, and assuming that the non-measured fraction is equal to the SM expectation, the final result for the fractions of longitudinally polarized and right handed $W$ bosons is:

$$
\begin{aligned}
& F_{0}=0.59 \pm 0.12 \text { (stat.) }{ }_{-0.06}^{+0.07} \text { (syst.) } \\
& F_{+}=-0.03 \pm 0.06 \text { (stat.) }{ }_{-0.03}^{+0.04} \text { (syst.) }
\end{aligned}
$$

We obtained an upper limit on the fraction of right handed $W$ bosons of $F_{+} \leq 0.10$ at the $95 \%$ C.L. Furthermore, our method provides the possibility to correct the observed $\cos \theta^{*}$ distribution for the selected sample for acceptance and resolution 
effects resulting in the distribution of the differential $t \bar{t}$ production cross section.

For the measurement of $F_{+}$the other parameter, $F_{0}$, was fixed to its SM value 0.7. Thus this measurement is a test on the anomalous right handed coupling $f_{1}^{R}$ and therefore on a possible $\mathrm{V}+\mathrm{A}$ component in the structure of the weak interaction in top quark decays. In the other case, $F_{0}$ was measured as single free parameter, while $F_{+}$was fixed to zero, and therefore the anomalous magnetic coupling $f_{2}^{R}$ under the assumption that no coupling to right handed $b$ quarks exist was tested. Both results were consistent with the SM prediction within the statistical uncertainties.

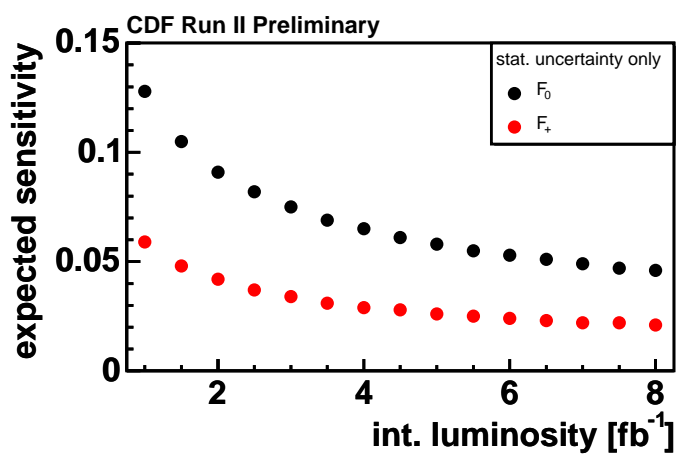

(a)

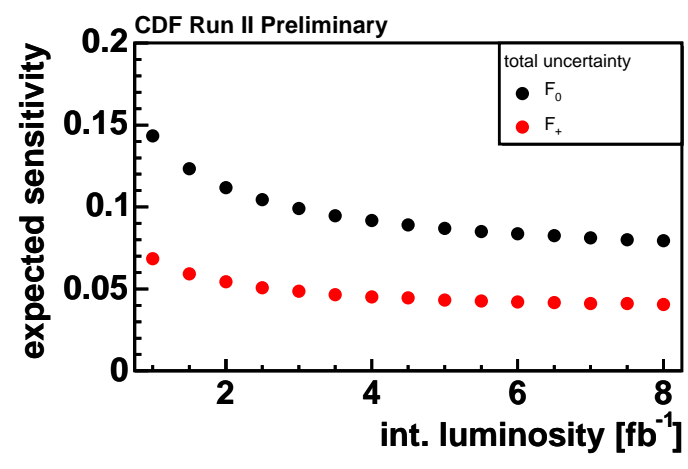

(b)

Figure 7.1: Expected sensitivities for higher integrated luminosities. In (a) only the statistical uncertainties are presented, while in (b) the total uncertainties are shown, obtained by adding statistical and systematic uncertainties in quadrature. For the systematic uncertainties the uncertainty of the measurement with $955 \mathrm{pb}^{-1}$ is taken. In both cases the upper black points represent the uncertainty for the measurement of $F_{0}$ and the lower red points represent the uncertainties for the measurement of $F_{+}$.

Though these uncertainties are equal to the uncertainties of the latest result for $F_{+}$and are significantly smaller than in previous measurements of $F_{0}$, the measurement is still statistically limited. Figure 7.1 shows the expected sensitivities for both measurements as a function of the integrated luminosity. In the near future, with higher statistics we will be able to set limits on the anomalous couplings, $f_{1}^{R} / f_{1}^{L}$ and $f_{2}^{R} / f_{1}^{L}$, for the two above mentioned non-SM scenarios. With more data collected and a finer binning in the $\cos \theta^{*}$ distribution it will also be possible to extract simultaneously both fractions $F_{0}$ and $F_{+}$with acceptable uncertainties to test models in which both fractions deviate from the SM values. 


\section{Bibliography}

[1] CDF Collaboration, F. Abe et al., Phys. Rev. Lett. 74, 2626 (1995).

[2] DØ Collaboration, S. Abachi et al., Phys. Rev. Lett. 74, 2632 (1995).

[3] T.E.W. Group (2006), hep-ex/0603039.

[4] A. Abulencia et al., Phys. Rev. Lett. 96, 022004 (2006).

[5] R.D. Peccei, S. Peris, and X. Zhang, Nucl. Phys. B 349, 305 (1991).

[6] D. Acosta et al., Phys. Rev. D 71, 031101 (2005).

[7] A. Abulencia et al., Phys. Rev. D 73, 111103 (2006).

[8] A. Abulencia et al.,(2006), hep-ex/0608062.

[9] A. Affolder et al., Phys. Rev. Lett. 84, 216 (2000).

[10] V.M. Abazov et al., Phys. Lett. B 617, 1 (2005).

[11] V.M. Abazov et al., Phys. Rev. D 72, 011104 (2005).

[12] S.L. Glashow, Nucl. Phys. 22, 579 (1961).

[13] S. Weinberg, Phys. Rev. Lett. 19, 1264 (1967).

[14] W.-M. Yao et al., J. Phys. G 33, 1 (2006).

[15] P.W. Higgs, Physics Lett. 12, 252 (1964).

[16] N. Cabbibo, Phys. Rev. Lett. 10, 531 (1963).

[17] M. Kobayashi and T. Maskawa, Prog. Theor. Phys. 49, 652 (1973).

[18] N. Kidonakis and R. Vogt, Phys. Rev. D 68, 114014 (2003).

[19] M. Cacciari et al., J. High Energy Phys. 0404, 068 (2004).

[20] G.L. Kane, G.A. Ladinsky, and C.P. Yuan, Phys. Rev. D 45, 124 (1992).

[21] F. Larios and C.-P. Yuan, Phys. Lett. B 457, 334 (1999), and references therin. 
[22] G. Burdman, M.C. Gonzalez-Garcia, and S.F. Novaes, Phys. Rev. D 61, 114016 (2000).

[23] Chuan-Ren Chen, F. Larios, and C.-P. Yuan, Phys. Lett. B 631, 126 (2005).

[24] J.M. Cornwall, D.N. Levin, and G. Tiktopoulos, Phys. Rev. D 10, 1145 (1974).

[25] B.W. Lee, C. Quigg, and H.B. Thacker, Phys. Rev. D 16, 1519 (1977).

[26] J.H. Kühn (1995), hep-ph/9707321.

[27] M. Jezabek and J.H. Kühn, Nucl. Phys. 314, 1 (1989).

[28] M. Jezabek and J.H. Kühn, Phys.Lett. 207, 91 (1988).

[29] A. Czarnecki and K. Melnikow, Nucl. Phys. 544, 520 (1999).

[30] K.G. Chetyrkin, R. Harlander, T. Seidensticker, and M. Steinhauser, Phys. Rev. 60, 114015 (1999).

[31] A. Denner and T. Sack, Nucl. Phys. 358, 46 (1991).

[32] R. Migneron, G. Eilam, R.R. Mendel, and A. Soni, Phys. Rev. Lett. 66, 3105 (1991).

[33] M. Fischer, S. Groote, J.G. Korner, M.C. Mauser, and B. Lampe, Phys. Lett. B 451, 406 (1999).

[34] M. Fischer, S. Groote, J.G. Korner, M.C. Mauser, Phys. Rev. D 63, 031501 (2001).

[35] M. Fischer, S. Groote, J.G. Korner, M.C. Mauser, Phys. Rev. D 65, 054036 (2002)

[36] H.S. Do, S. Groote, J.G. Korner, M.C. Mauser, Phys. Rev. D 67, 091501 (2003).

[37] J. Cao, R.J. Oakes, F. Wang, J.M. Yang, Phys. Rev. D 68, 054019 (2003).

[38] X. Wang, Q. Zhang, and Q.Qiao, Phys. Rev. D 71, 014035 (2005).

[39] R.D. Peccei, X. Zhang, Nucl. Phys. B 337, 269 (1990).

[40] P.H. Garbinicus, hep-ex/0406013.

[41] J. Farrington, " $H^{-}$Ion Beam Characterization", Fermilab Summer 2002 SIST Program.

[42] R.F. Welton, "Overview of High-Brightness $H^{-}$Ion Sources, Proceedings of LINAC 2002, Korea.

[43] CDF II Collaboration, FERMILAB-PUB-96/390-E (1996). 
[44] A. Sill et al., Nucl. Instrum. Meth. A 447, 1 (2000).

[45] A. Afforder et al., Nucl. Instrum. Meth. A 453, 84 (2000).

[46] C.S. Hill et al., Nucl. Instrum. Meth. A 511, 118 (2003).

[47] T. Affolder et al., Nucl. Instrum. Meth. A 526, 249 (2004).

[48] L. Balka et al., Nucl. Instrum. Meth. A 267, 272 (1988).

[49] S. Bertolucci et al., Nucl. Instrum. Meth. A 267, 301 (1988).

[50] M.G. Albrow et al., Nucl. Instrum. Meth. A 480, 524 (2002).

[51] A. Artikov et al., Nucl. Instrum. Meth. A 538, 358 (2000).

[52] M. Albrow, "CDF Run II Trigger Table and Dataset plan", CDF Internal Note No. 4718.

[53] B. Ashmanskas et al., Nucl. Instrum. Meth. A 518, 532 (2004).

[54] G. Gomez-Ceballos et al., Nucl. Instrum. Meth. A 518, 522 (2004).

[55] E. Sexton-Kennedy and P. Murat, eConf C0303241 (2003) THJT001, physics/0306112.

[56] W. Badgett, H. Frisch, R.St. Denis, T. Vaiciulis, "The Good Run List", Internal CDF Note No. 5613.

[57] Good Run List V13, http://www-cdf.fnal.gov/internal/dqm/goodrun/v13/goodv13.html

[58] T. Sjöstrand et al., Comput. Phys. Commun. 135, 238 (2001).

[59] HERWIG 6.5, G. Corcella, I.G. Knowles, G. Marchesini, S. Moretti, K. Odagiri, P. Richardson, M.H. Seymour and B.R. Webber, hep-ph/0011363, hep$\mathrm{ph} / 0210213$.

[60] "ALPGEN, a generator for hard multiparton processes in hadronic collisions", M.L. Mangano, M.Moretti, F. Piccinini, R. Pittau, A. Polesa, hep-ph/0206293.

[61] E. Gerchtein and M. Paulini, eConf C0303241 (2001) TUMT005.

[62] R. Erbacher et al.,"Event Selection and t-tbar Signal Acceptance of the Winter 2005 Top Lepton + Jets Sample", Internal CDF Note No 7372.

[63] C. Hill, J. Incandela, and C. Mills, "Electron Identification in Offline Release 5.3", Internal CDF Note No. 7309.

[64] V. Martin, "High $p_{\mathrm{T}}$ muons, recommended cuts and efficiencies for release 5.3.1", Internal CDF Note No. 7031. 
[65] B. Flaugher, J. Müller, "A Guide to JETCLU: The CDF Jet Cluster Algorithm", Internal CDF Note No. 1814.

[66] A.Bhatti et al., Nucl. Instrum. Meth. A 566, 375 (2006).

[67] C. Rott et al., "SecVtx Optimization for the 2003 Winter Conferences", Internal CDF Note No. 6242.

[68] Talk of Tatsuya Masabuchi, Top Properties Meeting 07/07/06.

[69] J.M. Campbell and R.K. Ellis, Phys. Rev, D 60, 113006 (1999).

[70] Z. Sullivan, Phys. Rev, D 70, 114012 (2004).

[71] B.W. Harris et al., Phys. Rev, D 66, 054024 (2002).

[72] D. Acosta et al., "Introduction to Run II Jet Probability Heavy Flavor Tagger", Internal CDF Note No. 6315.

[73] M. Feindt, S. Richter, W. Wagner, "A Neural Network b Tagger for Single-Top Analyses", Internal CDF Note No. 7816.

[74] V. Blobel and E. Lohrmann, "Statistische und numerische Methoden der Datenanalyse", Teubner, Stuttgart/Leipzig, 1998.

[75] A.D. Martin, R.G. Roberts, W.J. Stirling, and R.S. Thorne, Eur. Phys. J., C 4, 463 (1998).

[76] H.L. Lai et al., Eur. Phys. J., C 12, 375 (2000). 


\section{Danksagung}

An aller erster Stelle möchte ich mich bei Herrn Prof. Dr. Th. Müller dafür bedanken, dass er mich in seine Top-Quark-Gruppe aufgenommen hat und mir die Möglichkeit gegeben hat, während meiner Diplomarbeit für drei sehr interessante Wochen ans Fermilab zu fliegen um dort die Ergebnisse meiner Analyse vorzustellen.

Desweiteren bedanke ich mich bei Herrn Priv.-Doz. Dr. Wolfgang Wagner für die Übernahme des Korreferats für diese Arbeit sowie die hervorragende Betreuung in der Top-Gruppe und die sehr interessanten Tage am Fermilab in Chicago.

Außerdem danke ich meiner Betreuerin Dr. Jeannine Wagner für die unzähligen Tipps während der Analyse und das sehr aufmerksame Korrekturlesen dieser Arbeit sowie die damit verbundenen langen und ergiebigen Diskussionen.

Ein ganz besonders großes Dankeschön geht an Dr. Dominic Hirschbühl, auf dessen Arbeit diese Diplomarbeit aufbaut, und der mir während der gesamten Zeit, vom Beginn der Analyse bis zum letzten Kapitel dieser Arbeit stets mit Rat und Tat zur Seite stand.

Ich möchte der gesamten Karlsruher Top-Gruppe für die sehr angenehme Arbeitsatmosphäre und die vielen Tipps von allen Seiten danken. Dr. Yves Kemp danke ich für die Lösung vieler Computer-Probleme, Julia Weinelt für das sehr angenehme Arbeitsklima in unserem Büro sowie das Korrekturlesen dieser Arbeit. Svenja Richter, Adonis Papaikonomou und Jan Lück danke ich für die vielfältige und geduldige Hilfe und Unterstützung. Bei Matthias Bühler bedanke ich mich für seine Unterstützung und Freundschaft in den letzten fünf Jahren.

Weiterhin danke ich allen Mitgliedern des EKP im 8. und 9. Stockwerk sowie dem Sekretariat für die Zusammenarbeit und Hilfen vielfältiger Art. Auch dem gesamten Admin-Team sei herzlich für den unermüdlichen Einsatz, die Rechner am Laufen zu halten, gedankt.

Abschließend möchte ich meinen Eltern und Großeltern sowie meinen Geschwistern und Freunden danken, ohne deren Unterstützung diese Arbeit nicht möglich gewesen wäre. 
Hiermit versichere ich, die vorliegende Arbeit selbständig verfasst und nur die angegebenen Hilfsmittel verwendet zu haben.

Thorsten Chwalek

Karlsruhe, 30. Oktober 2006 